\title{
On the Persistence of Lower Dimensional Invariant Tori under Quasiperiodic Perturbations
}

\author{
Àngel Jorba* and Jordi Villanueva ${ }^{\dagger}$ \\ Departament de Matemàtica Aplicada I \\ Universitat Politècnica de Catalunya \\ Diagonal 647, 08028 Barcelona, Spain.
}

\begin{abstract}
In this work we consider time dependent quasiperiodic perturbations of autonomous Hamiltonian systems. We focus on the effect that this kind of perturbations has on lower dimensional invariant tori. Our results show that, under standard conditions of analyticity, nondegeneracy and nonresonance, most of these tori survive, adding the frequencies of the perturbation to the ones they already have.

The paper also contains estimates on the amount of surviving tori. The worst situation happens when the initial tori are normally elliptic. In this case, a torus (identified by the vector of intrinsic frequencies) can be continued with respect to a perturbative parameter $\varepsilon \in\left[0, \varepsilon_{0}\right]$, except for a set of $\varepsilon$ of measure exponentially small with $\varepsilon_{0}$. In case that $\varepsilon$ is fixed (and sufficiently small), we prove the existence of invariant tori for every vector of frequencies close to the one of the initial torus, except for a set of frequencies of measure exponentially small with the distance to the unperturbed torus. As a particular case, if the perturbation is autonomous, these results also give the same kind of estimates on the measure of destroyed tori.

Finally, these results are applied to some problems of celestial mechanics, in order to help in the description of the phase space of some concrete models.
\end{abstract}

* jorba@ma1.upc.es

† jordiotere. upc.es 


\section{Contents}

1 Introduction 3

2 Main ideas $\quad 5$

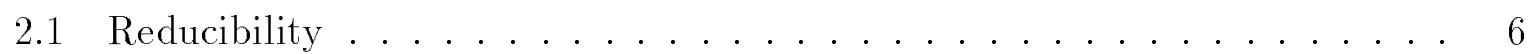

2.2 Normal form around the initial torus . . . . . . . . . . . . 7

2.3 The iterative scheme . . . . . . . . . . . . . . . 9

2.4 Estimates on the measure of preserved tori . . . . . . . . . . . . 12

2.5 Other parameters: families of lower dimensional tori . . . . . . . . . . 13

3 Statement of the results $\quad 14$

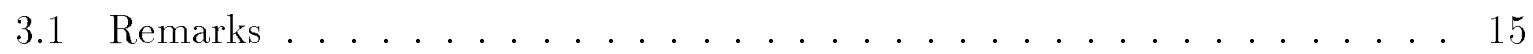

4 Applications $\quad \mathbf{1 5}$

4.1 The bicircular model near $L_{4,5} \ldots \ldots \ldots \ldots \ldots \ldots$. . . . . . . . 15

4.1 .1 Extensions . . . . . . . . . . . . . . . 17

4.2 Halo orbits . . . . . . . . . . . . . . . . . 17

5 Proofs $\quad \mathbf{1 8}$

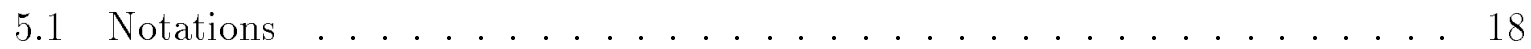

5.1.1 Norms and Lipschitz constants . . . . . . . . . . . . . . 18

5.1 .2 Canonical transformations . . . . . . . . . . . . . 19

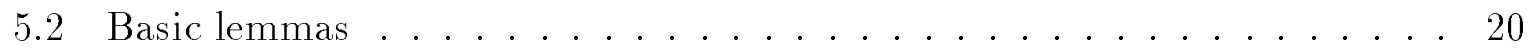

5.2 .1 Lemmas on norms and Lipschitz constants . . . . . . . . 20

5.2.2 Lemmas on canonical transformations . . . . . . . . . . . . . 24

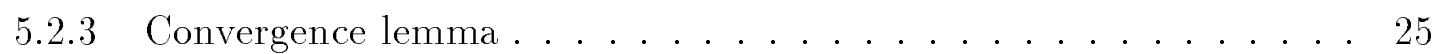

5.2.4 Lemmas on the control of the measure . . . . . . . . . . 25

5.3 Iterative lemma . . . . . . . . . . . . . . . . . . . 29

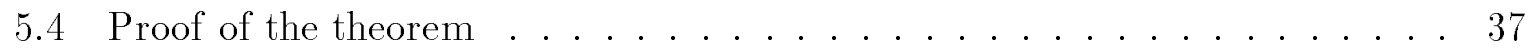

5.4 .1 Linear scheme with respect to $\varepsilon \ldots \ldots . \ldots . \ldots 37$

5.4 .2 Introduction of the vector of frequencies as a parameter . . . . . 38

5.4 .3 Inductive part . . . . . . . . . . . . . . . . 39

5.4 .4 Convergence of the changes of variables . . . . . . . . . . . . 41

5.4 .5 Control of the measure . . . . . . . . . . . . . . 43

$\begin{array}{lll}6 & \text { Acknowledgements } & 44\end{array}$

$\begin{array}{ll}\text { References } & 44\end{array}$ 


\section{Introduction}

Let $H$ be an autonomous Hamiltonian system with $\ell$ degrees of freedom, having the origin as an elliptic equilibrium point. If we take the linearization at this point as a first approximation to the dynamics, we see that all the solutions are quasiperiodic and can be described as the product of $\ell$ linear oscillators. The solutions of each oscillator can be parametrized by the amplitude of the orbits.

When the nonlinear part is added, each oscillator becomes a one parametric family of periodic orbits (usually called Lyapounov orbits), that can be still parametrized by the amplitude, at least near the origin (see [31]). Generically, the frequency of these orbits varies with their amplitude.

The effect that the nonlinear part of the Hamiltonian has on the quasiperiodic solutions is more complex. Without going into the details, KAM theorem states that under generic conditions of nonresonance on the frequencies of the oscillators and generic conditions of nondegeneracy on the nonlinear part of the Hamiltonian, most of these solutions still survive. Their frequencies now vary with the amplitude, and the measure of the destroyed tori is exponentially small with the distance to the origin (see [7]).

Usually these results are proved putting first the Hamiltonian into the more general form

$$
H=H_{0}(I)+H_{1}(\theta, I), \quad I=\left(I_{1}, \ldots, I_{\ell}\right), \quad \theta=\left(\theta_{1}, \ldots, \theta_{\ell}\right),
$$

where $H_{1}$ is small near the origin. This can be achieved, for instance, applying some steps of the process to put the Hamiltonian in (Birkhoff) normal form. If we neglect $H_{1}$, each quasiperiodic solution takes place on a torus $I=I^{*}$ with frequencies given by $\nabla H_{0}\left(I^{*}\right)$. Here the question is if these invariant tori are preserved when the perturbing term $H_{1}$ is added. The usual hypothesis are, essentially, two:

1. Nonresonance. The frequencies of the torus must satisfy a Diophantine condition:

$$
\left|k^{\top} \nabla H_{0}\left(I^{*}\right)\right| \geq \frac{\mu}{|k|_{1}^{\gamma}}, \quad \gamma>\ell-1
$$

where $k^{\top} \nabla H_{0}\left(I^{*}\right)$ denotes the scalar product of $k$ with the gradient of $H_{0}$, and $|k|_{1}=\left|k_{1}\right|+\cdots+\left|k_{\ell}\right|$.

2. Nondegeneracy. The frequencies must depend on the actions:

$$
\operatorname{det}\left(\frac{\partial^{2} H_{0}}{\partial I^{2}}\left(I^{*}\right)\right) \neq 0
$$

The necessity of the first hypothesis comes from the fact that, during the proof, we obtain the divisors $k^{\top} \nabla H_{0}\left(I^{*}\right)$. Hence, if they are too small it is not possible to prove the convergence of the series that appear in the proof (see [1] for the details).

An interesting case is when $k^{\top} \nabla H_{0}\left(I^{*}\right)$ is exactly zero, for some $k$. This implies that, as the frequencies are rationally dependent, the flow on the torus $I=I^{*}$ is not dense. More precisely, if one has $\ell_{i}$ independent frequencies, the torus $I=I^{*}$ contains an $\left(\ell-\ell_{i}\right)$-family of $\ell_{i}$-dimensional invariant tori, and each of these tori is densely filled up by the flow. Here the natural problem is also to study the persistence of these lower dimensional invariant tori when the nonintegrable part $H_{1}$ is taken into account. Generically, some of these tori 
survive but their normal behaviour can be either elliptic or hyperbolic (see [35], [25], [11], [25] and [19]). The invariant manifolds associated to the hyperbolic directions of these tori (usually called "whiskers") seem to be the skeleton that organizes the diffusion (see [2]).

Moreover, there are other families of lower dimensional tori that come from the Hamiltonian in normal form $H_{0}$. They can be obtained combining some of the elliptic directions associated to the fixed point, that is, they come from the product of some of the oscillators of the linearization. These tori are generically nonresonant, and some of them also survive when we add the nonitegrable part $H_{1}$ (see [9]). They are the generalitzation of the periodic Lyapounov orbits to higher dimensional tori and hence, we will call them Lyapounov tori.

In this paper we will focus on every kind of nondegenerate low dimensional torus, in the sense that its normal behaviour only contains elliptic or hyperbolic directions but not degenerate ones (see [17] and [36] for results in the hyperbolic case and [27], [9] and [28] for previous results in the general case). This implies that the torus is not contained in a (resonant) higher dimensional invariant torus.

We will develop a perturbation theory for these tori, focussing on the case in which the perturbation also depends on time in a quasiperiodic way. The Hamiltonian is of the form

$$
H(\theta, x, I, y)=\tilde{\omega}^{(0) \top} \tilde{I}+H_{0}(\hat{\theta}, x, \hat{I}, y)+H_{1}(\hat{\theta}, \tilde{\theta}, x, \hat{I}, y)
$$

with respect to the symplectic form $d \hat{\theta} \wedge d \hat{I}+d \tilde{\theta} \wedge d \tilde{I}+d x \wedge d y$. Here, $\hat{\theta}$ are the angular variables that describe an initial $r$-dimensional torus of $H_{0}, x$ and $y$ are the normal directions to the torus, $\tilde{\theta}$ are the angular variables that denotes the time, $\tilde{I}$ are the corresponding momenta (that has only been added to put the Hamiltonian in autonomous form) and $\tilde{\omega}^{(0)}$ is the frequency associated to time.

These kind of Hamiltonians appear in several problems of celestial mechanics: for instance, to study the dynamics of a small particle (an asteroid or spacecraft) near the equilateral libration points ([34]) of the Earth-Moon system, one can take the Earth-Moon system as a restricted three body problem (that can be written as an autonomous Hamiltonian) plus perturbations coming for the real motion of Earth and Moon and the presence of the Sun. As these perturbations can be very well approximated by quasiperiodic functions (at least for moderate time spans), it is usual to do so. Hence, one ends up with an autonomous model perturbed with a function that depends on time in a quasiperiodic way. Details on these models and their applications can be found in [8], [12], [14] and [16]. For more theoretical results, see [21], [23] and [20].

The problem of the preservation of maximal dimension tori of Hamiltonians like (1) has already been considered in [23]. There it is proved that most (in the usual measure sense) of the tori of the unperturbed system survive to the perturbation, but adding the perturbing frequencies to the ones they already have. Here we will consider the problem of the preservation of lower dimensional invariant tori, under the same kind of perturbations. We will show that, under some hypothesis of nondegeneracy and nonresonance (to be precised later) some of the (lower dimensional) tori are not destroyed but only deformed by the perturbation, adding the perturbing frequencies to the ones they previously had.

One of the main contributions of this paper are the estimates on the measure of the destroyed tori. We have taken two approaches to that point. In the first one we study the persistence of a single invariant torus of the initial Hamiltonian, under a quasiperiodic time-dependent perturbation, using as a parameter the size $(\varepsilon)$ of this perturbation. Our results show that this torus can be continued for a Cantor set of values of $\varepsilon$, adding the 
perturbing frequencies to the ones it already have. Moreover, if $\varepsilon \in\left[0, \varepsilon_{0}\right]$, the measure of the complementary of that Cantor set is exponentially small with $\varepsilon_{0}$. If the perturbation is autonomous this result is already contained in [19] but for 4-D symplectic maps.

The second approach is to fix the size of the pertubation to a given (and small enough) value. Then it is possible that the latter result can not be applied because $\varepsilon$ can be in the complementary of the above-defined Cantor set. In this case, it is still possible to prove the existence of invariant tori with frequencies the ones of the perturbation plus frequencies close to the ones of the unperturbed torus. These tori are a Cantor family parametrized (for instance) by the frequencies of the unperturbed problem. Again, the measure of the complementary of this Cantor set is exponentially small with the distance to the frequencies of the initial torus.

It is interesting to note the implications of this last assertion when the perturbation is autonomous and the size of the perturbation is fixed: in this case we are proving, for the perturbed Hamiltonian, the existence of a Cantor family of invariant tori near the initial one (see [9] and [33]). Moreover, the measure of the complementary of this set is exponentially small with the distance to the initial tori.

The most difficult case is when the normal behaviour of the torus contain some elliptic directions, because the (small) divisors obtained contain combinations of the intrinsic frequencies with the normal ones. As we will see, it is not difficult to control the value of the intrinsic frequencies but then we have no control (in principle) on the corresponding normal ones. This is equivalent to say that we can not select a torus with given both intrinsic and normal frequencies, because there are not enough available parameters (see [27], [4] and [33]). The main trick in the proofs is to assume that the normal frequencies move as a function of $\varepsilon$ (then we derive the existence of the torus for a Cantor set of $\varepsilon$ ) or as a function of the intrinsic frequencies (then we obtain the existence of the above-mentioned family of tori, close to the initial one).

When the initial torus is normally hyperbolic we do not need to control the eigenvalues in the normal direction and, hence, we do not have to deal with the lack of parameters. Of course, in this case the results are much better and the proofs can be seen as simplifications of the ones contained here. Hence, this case is not explicity considered.

Finally, we have also included examples where the application of these results helps to understand the dynamics of concrete problems.

The paper has been organized as follows: section 2 contains the main ideas used to derive these results. Section 3 contains the rigorous statement of the results. The applications of these results to some concrete problems can be found in section 4 and, finally, section 5 contains the technical details of the proofs.

\section{Main ideas}

Let $\mathcal{H}$ be a Hamiltonian system of $\ell$ degrees of freedom in $\mathbb{C}^{2 \ell}$ having an invariant $r$ dimensional torus, $0 \leq r \leq \ell$, with a quasiperiodic flow given by the vector of basic frequencies $\hat{\omega}^{(0)} \in \mathbb{R}^{r}$. Let us consider the (perturbed) Hamiltonian system $H=\mathcal{H}+\varepsilon \hat{\mathcal{H}}$. As it has been mentioned before, we do not restrict ourselves to the case of autonomous perturbations, but we will assume that $\hat{\mathcal{H}}$ depends on time in a quasiperiodic way, with vector of basic frequencies given by $\tilde{\omega}^{(0)} \in \mathbb{R}^{s}$. 


\subsection{Reducibility}

Let us consider the variational flow around one of the quasiperiodic orbits of the initial $r$-dimensional invariant torus of $\mathcal{H}$. The variational equations are a linear system with quasiperiodic time dependence, with vector of basic frequencies $\hat{\omega}^{(0)}$. When the torus is a periodic orbit, the well know Floquet theorem states that we can reduce this periodic system to constant coefficients with a linear periodic change of variables (with the same period of the system). This change can be selected to be canonical if the equations are Hamiltonian. So, the reduced matrix has a pair of zero eigenvalues (associated to the tangent vector to the periodic orbit) plus eigenvalues that describes the linear normal behaviour around the torus. We will assume that these eigenvalues are all different (this condition implies, from the canonical character of the system, that they are also non-zero). This implies that the periodic orbit is not contained in a (resonant) higher dimensional torus. Usually, the imaginary parts of these eigenvalues are called normal frequencies, and $\hat{\omega}^{(0)}$ is called the vector of intrinsic frequencies of the torus.

The quasiperiodic case $(r>1)$ is more complex, because we can not guarantee in general the reducibility to constant coefficients of the variational equations with a linear quasiperiodic change of variables with the same basic frequencies as the initial system. The question of reducibility of linear quasiperiodic systems (proved in some cases, see [18], [5], [10], [21], [23], [19], and [24], among others) remains open in the general case. However, we can say that if this reduction is possible, we have $2 r$ zero eigenvalues (related to the $r$ tangent vectors to the torus).

Here we will assume that such reduction is possible for the initial torus. We want to remark that if this initial torus comes from an autonomous perturbation of a resonant torus of an integrable Hamiltonian, this hypothesis is not very strong. To justify this assertion, we mention the following fact: let us write the Hamiltonian as $H=\mathcal{H}(I)+\varepsilon \hat{\mathcal{H}}(\theta, I)$, and let $\mathcal{T}_{0}$ be a low dimensional invariant tori of the integrable Hamiltonian $\mathcal{H}(I)$ that survives to the perturbation $\varepsilon \hat{\mathcal{H}}(\theta, I)$. Then, under generic hypothesis of nondegeneracy and nonresonance, this low dimensional torus exists and its normal flow is also reducible for a Cantor set of values of $\varepsilon$. The Lebesgue measure of the complementary of this set in $\left[0, \varepsilon_{0}\right]$ is exponentially small with $\varepsilon_{0}$. This fact is proved for symplectic diffeomorphisms of $\mathbb{R}^{4}$ in [19], but it is immediate to extend to other cases.

Moreover, let us assume that we can introduce (with a canonical change of coordinates) $r$ angular variables $\hat{\theta}$ describing the initial torus. Hence, the Hamiltonian takes the form

$$
\mathcal{H}(\hat{\theta}, x, \hat{I}, y)=\hat{\omega}^{(0) \top} \hat{I}+\frac{1}{2} z^{\top} \mathcal{B} z+\mathcal{H}_{*}(\hat{\theta}, x, \hat{I}, y)
$$

where $z^{\top}=\left(x^{\top}, y^{\top}\right)$, being $z, \hat{\theta}$ and $\hat{I}$ complex vectors, $x$ and $y$ elements of $\mathbb{C}^{r}$ and $\hat{\theta}$ and $\hat{I}$ elements of $\mathbb{C}^{s}$, with $r+m=\ell$. Here, $\hat{\theta}$ and $x$ are the positions and $\hat{I}$ and $y$ are the conjugate momenta. In this notation $\mathcal{B}$ is a symmetric $2 m$-dimensional matrix (with complex coefficients). Moreover, $\mathcal{H}_{*}$ is an analytic function (with respect to all its arguments) with $2 \pi$-periodic dependence on $\hat{\theta}$. More concretely, we will assume that it is analytic on a neighbourhood of $z=0, \hat{I}=0$, and on a complex strip of positive width $\rho$ for the variable $\hat{\theta}$, that is, if $\left|\operatorname{Im} \hat{\theta}_{j}\right| \leq \rho$, for all $j=1, \ldots, r$. Then, if we assume that $\mathcal{H}$ has an invariant $r$-dimensional torus with vector of basic frequencies $\hat{\omega}^{(0)}$, given by $\hat{I}=0$ and $z=0$, this implies that the Taylor expansion of $\mathcal{H}_{*}$ must begin with terms of second order in the variables $\hat{I}$ and $z$. If we have that the normal variational flow around this 
torus can be reduced to constant coefficients, we can assume that the quadratic terms of $\mathcal{H}_{*}$ in the $z$ variables vanish. Hence, the normal variational equations are given by the matrix $J_{m} \mathcal{B}$, where $J_{m}$ is the canonical 2-form of $\mathbb{C}^{2 m}$. We also assume that the matrix $J_{m} \mathcal{B}$ is in diagonal form with different eigenvalues $\lambda^{\top}=\left(\lambda_{1}, \ldots, \lambda_{m},-\lambda_{1}, \ldots,-\lambda_{m}\right)$.

Let us give some remarks on these coordinates. First, note that we have assumed that the initial torus is isotropic (this is, the canonical 2-form of $\mathbb{C}^{2 \ell}$ restricted to the tangent bundle of the torus vanishes everywhere). This fact (that is always true for a periodic orbit) is not a strong assumption for a torus, because all the tori obtained by applying KAM techniques to near-integrable Hamiltonian systems are isotropic.

Another point worth to comment is the real or complex character of the matrix $\mathcal{B}$. In this paper we work, in principle, with complex analytic Hamiltonian systems, but the most interesting case happens when we deal with real analytic ones, and when the initial torus is also real. In this case, to guarantee that the perturbative scheme preserves the real character of the tori, we want that the initial reduced matrix $\mathcal{B}$ comes from a real matrix. We note that this is equivalent to assume that if $\lambda$ is an eigenvalue of $J_{m} \mathcal{B}$, then $\bar{\lambda}$ is also an eigenvalue. This assumption is not true in general for every reducible torus of a real analytic Hamiltonian system, but it holds for most of the tori one can obtain near an initial torus with a normal flow reducible over $\mathbb{R}$. Note that in the case of a periodic orbit one can always assume that $\mathcal{B}$ is real (doubling the period if necessary). The fact that $\mathcal{B}$ is real guarantees that all the tori obtained are also real. To see it, we note that we can use the same proof but putting $J_{m} \mathcal{B}$ in real normal form instead of diagonal form, and this makes that all the steps of the proof are also real. However, the technical details in this case are a little more tedious and, hence, we have prefered to work with a diagonal $J_{m} \mathcal{B}$.

\subsection{Normal form around the initial torus}

The first step is to rearrange the initial Hamiltonian $\mathcal{H}^{(0)}:=\mathcal{H}$ in a suitable form to apply an inductive procedure.

In what follows, we will define degree of a monomial $z^{l} \hat{I}^{j}$ as $|l|_{1}+2|j|_{1}$. This definition is motivated below. Let us expand $\mathcal{H}_{*}^{(0)}$ in power series with respect to $z$ and $\hat{I}$ around the origin:

$$
\mathcal{H}_{*}^{(0)}=\sum_{d \geq 2} \mathcal{H}_{d}^{(0)}
$$

where $\mathcal{H}_{d}^{(0)}$ are homogeneous polynomials of degree $d$, that is,

$$
\mathcal{H}_{d}^{(0)}=\sum_{\substack{l \in \mathbb{N}^{2 m}, j \in \mathbb{N}^{r} \\|l|_{1}+2|j|_{1}=d}} h_{l, j}^{(0)}(\hat{\theta}) z^{l} \hat{I}^{j} .
$$

We also expand the (periodic) coefficients in Fourier series:

$$
h_{l, j}^{(0)}(\hat{\theta})=\sum_{k \in \mathbb{Z}^{r}} h_{l, j, k}^{(0)} \exp \left(i k^{\top} \hat{\theta}\right)
$$

being $i=\sqrt{-1}$. The definition of degree for a monomial $z^{l} \hat{I}^{j}$ counting twice the contribution of the variable $\hat{I}$ is motivated by the definition of the Poisson bracket of two functions 
depending on $(\hat{\theta}, x, \hat{I}, y)$ :

$$
\{f, g\}=\frac{\partial f}{\partial \hat{\theta}}\left(\frac{\partial g}{\partial \hat{I}}\right)^{\top}-\frac{\partial f}{\partial \hat{I}}\left(\frac{\partial g}{\partial \hat{\theta}}\right)^{\top}+\frac{\partial f}{\partial z} J_{m}\left(\frac{\partial g}{\partial z}\right)^{\top}
$$

Note that, if $f$ is an homogeneous polynomial of degree $d_{1}$ and $g$ is an homogeneous polynomial of degree $d_{2}$ then $\{f, g\}$ is an homogeneous polynomial of degree $d_{1}+d_{2}-2$. This property shows that if we try to construct canonical changes using the Lie series method, the adequate form to put $\mathcal{H}^{(0)}$ in normal form is to remove in an increasing order the terms of degree $3,4, \ldots$, with a suitable generating function.

To introduce some of the parameters (see section 2.5), it is very convenient that the initial Hamiltonian has the following properties:

P1 The coefficients of the monomials $(z, \hat{I})$ (degree 3$)$ and $(z, \hat{I}, \hat{I})$ (degree 5$)$ are zero.

P2 The coefficients of the monomials $(z, z, \hat{I})$ (degree 4 ) and $(\hat{I}, \hat{I})$ (degree 4$)$ do not depend on $\hat{\theta}$ and, in the case of $(z, z, \hat{I})$, they vanish except for the coefficients of the trivial resonant terms.

Here, we have used the following notation: for instance, by the terms of order $(z, z, \hat{I})$ we denote the monomials $z^{l} \hat{I}^{j}$, with $|l|_{1}=2$ and $|j|_{1}=1$, with the corresponding coefficients. We will apply three steps of a normal form procedure in order to achieve these conditions. Each step is done using a generating function of the following type:

$$
S^{(n)}(\hat{\theta}, x, \hat{I}, y)=\sum_{\substack{l \in \mathbb{N}^{2 m}, j \in \mathbb{N}^{r} \\\left|l l_{1}+2\right| j||_{1}=n}} s_{l, j}^{(n)}(\hat{\theta}) z^{l} \hat{l}^{j},
$$

for $n=3,4$ and 5 . Then, if we put $\Psi^{S^{(n)}}$ for the flow at time one of the Hamiltonian system associated to $S^{(n)}$, we transform the initial Hamiltonian into

$$
\begin{aligned}
\mathcal{H}^{(n-2)} & =\mathcal{H}^{(n-3)} \circ \Psi^{S^{(n)}}= \\
& =\mathcal{H}^{(n-3)}+\left\{\mathcal{H}^{(n-3)}, S^{(n)}\right\}+\frac{1}{2 !}\left\{\left\{\mathcal{H}^{(n-3)}, S^{(n)}\right\}, S^{(n)}\right\}+O_{n+1}= \\
& =\hat{\omega}^{(0) \top} \hat{I}+\frac{1}{2} z^{\top} \mathcal{B} z+\mathcal{H}_{*}^{(n-3)}+\left\{\hat{\omega}^{(0) \top} \hat{I}+\frac{1}{2} z^{\top} \mathcal{B} z, S^{(n)}\right\}+O_{n+1},
\end{aligned}
$$

for $n=3,4,5$. In each step, we take $S^{(n)}$ such that $\mathcal{H}_{n}^{(n-3)}+\left\{\hat{\omega}^{(0) \top} \hat{I}+\frac{1}{2} z^{\top} \mathcal{B} z, S^{(n)}\right\}$ satisfies conditions P1 and P2 for the monomials of degree $n(n=3,4,5)$. To compute $S^{(n)}$ we expand $\mathcal{H}_{n}^{(n-3)}$ and we find (formally) an expansion for $S^{(n)}$ :

$$
s_{l, j, k}^{(n)}=\frac{h_{l, j, k}^{(n-3)}}{i k^{\top} \hat{\omega}^{(0)}+l^{\top} \lambda},
$$

where the indices have the same meaning as in (2). If we split $l=\left(l_{x}, l_{y}\right)\left(z^{l}=x^{l_{x}} y^{l_{y}}\right)$, the exactly resonant terms correspond to $k=0$ and $l_{x}=l_{y}$ (we recall that $\lambda^{\top}=$ $\left.\left(\lambda_{1}, \ldots, \lambda_{m},-\lambda_{1}, \ldots,-\lambda_{m}\right)\right)$. Hence, it would be possible to formally compute a normal form depending only on $\hat{I}$ and the products $x_{j} y_{j}, j=1, \ldots, m$. As it has been mentioned before, our purpose is much more modest. To kill the monomials mentioned above (in 
conditions P1 and P2) with a convergent change of variables, one needs a condition on the smallness of $\left|i k^{\top} \hat{\omega}^{(0)}+l^{\top} \lambda\right|, k \in \mathbb{Z}^{r} \backslash\{0\}, l \in \mathbb{N}^{2 m}$ and $|l|_{1} \leq 2$. We have used the usual one

$$
\left|i k^{\top} \hat{\omega}^{(0)}+l^{\top} \lambda\right| \geq \frac{\mu_{0}}{|k|_{1}^{\gamma}},
$$

that we will assume true in the statement of the results. We notice that with these conditions we can construct convergent expressions for the different generating functions $S^{(n)}$, $n=3,4,5$, to achieve conditions $\mathbf{P} \mathbf{1}$ and $\mathbf{P} 2$. We can call this process a seminormal form construction.

Then, the final form for the Hamiltonian is

$$
\mathcal{H}=\hat{\omega}^{(0) \top} \hat{I}+\frac{1}{2} z^{\top} \mathcal{B} z+\frac{1}{2} \hat{I}^{\top} \mathcal{C} \hat{I}+H_{*}(\hat{\theta}, x, \hat{I}, y),
$$

for which conditions $\mathbf{P} \mathbf{1}$ and $\mathbf{P} \mathbf{2}$ holds. Here $\mathcal{C}$ is a symmetric constant matrix and we will assume $\operatorname{det} \mathcal{C} \neq 0$ (this is one of the nondegeneracy hypothesis). Now let us introduce the quasiperiodic time-dependent perturbation. To simplify the notation, we write this perturbation in the normal form variables, and we add this perturbation to (3). We call $H$ to the new Hamiltonian:

$$
H(\theta, x, I, y, \varepsilon)=\omega^{(0) \top} I+\frac{1}{2} z^{\top} \mathcal{B} z+\frac{1}{2} \hat{I}^{\top} \mathcal{C} \hat{I}+H_{*}(\hat{\theta}, x, \hat{I}, y)+\varepsilon \hat{\mathcal{H}}(\theta, x, \hat{I}, y, \varepsilon),
$$

for a fixed $\omega^{(0) \top}=\left(\hat{\omega}^{(0) \top}, \tilde{\omega}^{(0) \top}\right), \omega^{(0)} \in \mathbb{R}^{r+s}$, where $\theta^{\top}=\left(\hat{\theta}^{\top}, \tilde{\theta}^{\top}\right), I^{\top}=\left(\hat{I}^{\top}, \tilde{I}^{\top}\right)$ and $z^{\top}=\left(x^{\top}, y^{\top}\right)$, being $\tilde{\theta}, \tilde{I}$ (s-dimensional complex vectors) the new positions and momenta added to put in autonomous form the quasiperiodic perturbation. Hence, $H$ is $2 \pi$-periodic in $\theta$. Moreover, $\varepsilon$ is a small positive parameter. This is the Hamiltonian that we consider in the formulation of the results.

\subsection{The iterative scheme}

Before the explicit formulation of the results, let us describe a generic step of the iterative method used in the proof. So, let us consider a Hamiltonian of the form:

$$
H(\theta, x, I, y)=\omega^{(0) \top} I+\frac{1}{2} z^{\top} \mathcal{B} z+\frac{1}{2} \hat{I}^{\top} \mathcal{C}(\theta) \hat{I}+H_{*}(\theta, x, \hat{I}, y)+\varepsilon \hat{H}(\theta, x, \hat{I}, y),
$$

with the same notations of (4), where we assume that skipping the term $\varepsilon \hat{H}$, we have that $z=0, \hat{I}=0$ is a reducible $(r+s)$-dimensional torus with vector of basic frequencies $\omega^{(0)}$, such that the variational normal flow is given by $J_{m} \mathcal{B}=\operatorname{diag}\left(\lambda_{1}, \ldots, \lambda_{m},-\lambda_{1}, \ldots,-\lambda_{m}\right)$, and that $\operatorname{det} \overline{\mathcal{C}} \neq 0$, where $\overline{\mathcal{C}}$ means the average of $\mathcal{C}$ with respect to its angular variables (although initially $\mathcal{C}$ does not depend on $\theta$, during the iterative scheme it will). Moreover, we suppose that in $H_{*}$ the terms of order $(\hat{I}, z)$ vanish (that is, we suppose that the "central" and "normal" directions of the unperturbed torus have been uncoupled up to first order). Here we only use the parameter $\varepsilon$ to show that the perturbation $\varepsilon \hat{H}$ is of $O(\varepsilon)$.

We expand $\hat{H}$ in power series around $\hat{I}=0, z=0$ and we add these terms to the previous expansion of the unperturbed Hamiltonian. This makes that the initial torus is not longer invariant. Hence, the expression of the Hamiltonian must be (without writting explicity the dependence on $\varepsilon$ ):

$$
H(\theta, x, I, y)=\tilde{\omega}^{(0) \top} \tilde{I}+H^{*}(\theta, x, \hat{I}, y),
$$


where

$$
H^{*}=a(\theta)+b(\theta)^{\top} z+c(\theta)^{\top} \hat{I}+\frac{1}{2} z^{\top} B(\theta) z+\hat{I}^{\top} E(\theta) z+\frac{1}{2} \hat{I}^{\top} C(\theta) \hat{I}+\Omega(\theta, x, \hat{I}, y),
$$

being $\Omega$ the remainder of the expansion. Looking at this expression, we introduce the notation $\left[H^{*}\right]_{(z, z)}=B,\left[H^{*}\right]_{(\hat{I}, \hat{I})}=C,\left[H^{*}\right]_{(\hat{I}, z)}=E$ and $\left\langle H^{*}\right\rangle=H^{*}-\Omega$.

We have that $\tilde{a}, b, c-\hat{\omega}^{(0)}, B-\mathcal{B}, C-\mathcal{C}$ and $E$ are $O(\varepsilon)$, where if $f(\theta)$ is a periodic function on $\theta, \tilde{f}=f-\bar{f}$.

Note that if we are able to kill the terms $\tilde{a}, b$ and $c-\hat{\omega}^{(0)}$ we obtain a lower dimensional invariant torus with intrinsic frequency $\omega^{(0)}$. We will try to do that by using a quadratically convergent scheme. As it is usual in this kind of Newton methods, it is very convenient to kill something more. Before continuing, let us introduce the following notation: if $A$ is a $n \times n$ matrix, $\operatorname{dp}(A)$ denotes the diagonal part of $A$, that is, $\operatorname{dp}(A)=\operatorname{diag}\left(a_{1,1}, \ldots, a_{n, n}\right)^{\top}$, where $a_{i, i}$ are the diagonal entries of $A$. Here, we want that the new matrix $B$ verifies $B=\mathcal{J}_{m}(B)$, where we define $\mathcal{J}_{m}(B)=-J_{m} \mathrm{dp}\left(J_{m} \bar{B}\right)$ (this is, we ask the normal flow to the torus to be reducible and given by a diagonal matrix like for the unperturbed torus) and to eliminate $E$ (to uncouple the "central" and the normal directions of the torus up to first order in $\varepsilon$ ). Hence, the torus we will obtain has also these two properties. This is a very usual technique (see [9], [19]).

At each step of the iterative procedure, we use a canonical change of variables similar to the ones used in [3] to prove the Kolmogorov theorem. The generating function is of the form

$$
S(\theta, x, \hat{I}, y)=\xi^{\top} \hat{\theta}+d(\theta)+e(\theta)^{\top} z+f(\theta)^{\top} \hat{I}+\frac{1}{2} z^{\top} G(\theta) z+\hat{I}^{\top} F(\theta) z,
$$

where $\xi \in \mathbb{C}^{r}, \bar{d}=0, \bar{f}=0$ and $\mathrm{G}$ is a symmetric matrix with $\mathcal{J}_{m}(G)=0$. Keeping the same name for the new variables, the transformed Hamiltonian is

$$
H^{(1)}=H \circ \Psi^{S}=\tilde{\omega}^{(0) \top} \tilde{I}+H^{(1) *}(\theta, x, \hat{I}, y),
$$

being

$$
\begin{aligned}
H^{(1) *}(\theta, x, \hat{I}, y)= & a^{(1)}(\theta)+b^{(1)}(\theta)^{\top} z+c^{(1)}(\theta)^{\top} \hat{I}+\frac{1}{2} z^{\top} B^{(1)}(\theta) z+ \\
& +\hat{I}^{\top} E^{(1)}(\theta) z+\frac{1}{2} \hat{I}^{\top} C^{(1)}(\theta) \hat{I}+\Omega^{(1)}(\theta, x, \hat{I}, y) .
\end{aligned}
$$

We want $\tilde{a}^{(1)}=0, b^{(1)}=0, c^{(1)}-\hat{\omega}^{(0)}=0, E^{(1)}=0$ and $J_{m} B^{(1)}$ to be a constant diagonal matrix. We will show that this can be achieved up to first order in $\varepsilon$. So, we write those conditions in terms of the initial Hamiltonian and the generating function. Skipping terms of $\mathrm{O}_{2}(\varepsilon)$, we obtain:

$\left(e q_{1}\right) \tilde{a}-\frac{\partial d}{\partial \theta} \omega^{(0)}=0$,

$\left(e q_{2}\right) b-\frac{\partial e}{\partial \theta} \omega^{(0)}+\mathcal{B} J_{m} e=0$,

$\left(e q_{3}\right) c-\hat{\omega}^{(0)}-\frac{\partial f}{\partial \theta} \omega^{(0)}-\mathcal{C}\left(\xi+\left(\frac{\partial d}{\partial \hat{\theta}}\right)^{\top}\right)=0$,

$\left(e q_{4}\right) B^{*}-\mathcal{J}_{m}\left(B^{*}\right)-\frac{\partial G}{\partial \theta} \omega^{(0)}+\mathcal{B} J_{m} G-G J_{m} \mathcal{B}=0$, 
$\left(e q_{5}\right) E^{*}-\frac{\partial F}{\partial \theta} \omega^{(0)}-F J_{m} \mathcal{B}=0$,

being

$$
B^{*}=B-\left[\frac{\partial H_{*}}{\partial \hat{I}}\left(\xi+\left(\frac{\partial d}{\partial \hat{\theta}}\right)^{\top}\right)-\frac{\partial H_{*}}{\partial z} J_{m} \epsilon\right]_{(z, z)}
$$

and

$$
E^{*}=E-\mathcal{C}\left(\frac{\partial e}{\partial \hat{\theta}}\right)^{\top}-\left[\frac{\partial H_{*}}{\partial \hat{I}}\left(\xi+\left(\frac{\partial d}{\partial \hat{\theta}}\right)^{\top}\right)-\frac{\partial H_{*}}{\partial z} J_{m} e\right]_{(\hat{I}, z)}
$$

Here we denote by $\frac{\partial}{\partial q}$ the matrix of partial derivatives with respect to the variables $q$ and, for instance, $\frac{\partial G}{\partial \theta} \omega^{(0)}$ means $\sum_{j=1}^{r+s} \frac{\partial G}{\partial \theta_{j}} \omega_{j}^{(0)}$. These equations are solved formally by expanding in Fourier series and equating the corresponding coefficients. This lead us to the following expressions for $S$ :

$\left(e q_{1}\right)$

$$
d(\theta)=\sum_{k \in \mathbb{Z}^{r+s} \backslash\{0\}} \frac{a_{k}}{i k^{\top} \omega^{(0)}} \exp \left(i k^{\top} \theta\right) .
$$

$\left(e q_{2}\right)$ If we put $e^{\top}=\left(e_{1}, \ldots, e_{2 m}\right)$,

$$
e_{j}(\theta)=\sum_{k \in \mathbb{Z}^{r+s}} \frac{b_{j, k}}{i k^{\top} \omega^{(0)}+\lambda_{j}} \exp \left(i k^{\top} \theta\right) .
$$

$\left(e q_{3}\right)$

$$
\xi=(\overline{\mathcal{C}})^{-1}\left(\bar{c}-\hat{\omega}^{(0)}-\overline{\mathcal{C}\left(\frac{\partial d}{\partial \hat{\theta}}\right)^{\top}}\right)
$$

and if we define

$$
c^{*}=\tilde{c}-\tilde{\mathcal{C}} \xi-\mathcal{C}\left(\frac{\partial d}{\partial \hat{\theta}}\right)^{\top}+\overline{\mathcal{C}\left(\frac{\partial d}{\partial \hat{\theta}}\right)^{\top}}
$$

we have for $f^{\top}=\left(f_{1}, \ldots, f_{r}\right)$ :

$$
f_{j}(\theta)=\sum_{k \in \mathbb{Z}^{r+s} \backslash\{0\}} \frac{c_{j, k}^{*}}{i k^{\top} \omega^{(0)}} \exp \left(i k^{\top} \theta\right) .
$$

$\left(e q_{4}\right)$ If we define

$$
B^{* *}=B^{*}-\mathcal{J}_{m}\left(B^{*}\right),
$$

then we have for $G=\left(G_{j, l}\right), 1 \leq j, l \leq 2 m$,

$$
G_{j, l}(\theta)=\sum_{k \in \mathbb{Z}^{r+s}} \frac{B_{j, l, k}^{* *}}{i k^{\top} \omega^{(0)}+\lambda_{j}+\lambda_{l}} \exp \left(i k^{\top} \theta\right), \quad j, l=1, \ldots, 2 m .
$$

In the definition of $G_{j, l}$, we notice that we have trivial zero divisors when $|j-l|=m$ and $k=0$, but from the expression of $B^{* *}$, in these cases the coefficient $B_{j, l, 0}^{* *}$ is 0 . Moreover, the matrix $G$ is symmetric. 
$\left(e q_{5}\right)$ If $F=\left(F_{j, l}\right), j=1, \ldots, r$ and $l=1, \ldots, 2 m$, then

$$
F_{j, l}(\theta)=\sum_{k \in \mathbb{Z}^{r+s}} \frac{E_{j, l, k}^{*}}{i k^{\top} \omega^{(0)}+\lambda_{l}} \exp \left(i k^{\top} \theta\right)
$$

Note that if we have Diophantine hypothesis on the small divisors of these expressions,

$$
\left|i k^{\top} \omega^{(0)}+l^{\top} \lambda\right| \geq \frac{\mu_{0}}{|k|_{1}^{\gamma}}, \quad k \in \mathbb{Z}^{r+s} \backslash\{0\}, \quad l \in \mathbb{N}^{2 m}, \quad|l|_{1} \leq 2, \quad \gamma>r+s-1,
$$

we can guarantee the convergence of the expansion of $S$. We assume that they hold in the first step, and we want to have similar conditions after each step of the process, to be able to iterate. As the frequencies $\tilde{\omega}^{(0)}$ are fixed in all the process and $\hat{\omega}^{(0)}$ can be preserved by the nondegeneracy and the kind of generating function we are using (this is done by the $\xi$ term), we will be able to recover the Diophantine properties on them. The main problem are the eigenvalues $\lambda$, because, in principle, we can not preserve their value. Hence, we will control the way they vary, to try to ensure they are still satisfying a good Diophantine condition. Our first approach is to consider $\lambda$ as a function of $\varepsilon$ (the size of the perturbation). This leads us to eliminate a Cantor set of values of these parameters in order to have all the time good (in a Diophantine sense) values of $\lambda$. Another possibility is to consider $\lambda$ as a function of the (frequencies of the) torus. This leads us to eliminate a Cantor set of those tori.

\subsection{Estimates on the measure of preserved tori}

The technique we are going to apply to produce exponentially small estimates has already been used in [23]. It is based on working at every step $n$ of the iterative procedure with values of $\varepsilon$ for which we have Diophantine conditions of the type

$$
\left|i k^{\top} \omega^{(0)}+\lambda_{l}^{(n)}(\varepsilon)\right| \geq \frac{\mu_{n}}{|k|_{1}^{\gamma}} \exp \left(-\delta_{n}|k|_{1}\right), \quad k \in \mathbb{Z}^{r+s} \backslash\{0\},
$$

where $\lambda_{l}^{(n)}(\varepsilon)$ denotes the eigevalues of $J_{m} \mathcal{B}^{(n)}(\varepsilon)$, being $\mathcal{B}^{(n)}(\varepsilon)$ the matrix that replaces $\mathcal{B}$ after $n$ steps of the iterative process. Of course, we ask for the same condition for the sum of eigenvalues $\lambda_{j}^{(n)}+\lambda_{l}^{(n)}$. We will see that, if we take a suitable sequence of $\delta_{n}$, the exponential term in (12) is not an obstruction to the convergence of the scheme. This condition will be used to obtain exponentially small estimates for the measure of the values of $\varepsilon$ for which we do not have invariant tori of frequency $\omega^{(0)}$ in the perturbed system. The key idea can be described as follows: for the values of $\varepsilon$ for which we can prove convergence, we obviously have that, if $\varepsilon$ is small enough, $\left|\lambda_{l}^{(n)}(\varepsilon)-\lambda_{l}\right| \leq a \varepsilon$, at every step $n$. Now, if we assume that $\mu_{n} \leq \mu_{0} / 2$, from the Diophantine bounds on $i k^{\top} \omega^{(0)}+\lambda_{l}$ in (11), we only need to worry about the resonances corresponding to values of $k$ such that

$$
|k|_{1} \geq\left(\frac{\mu_{0}}{2 a \varepsilon}\right)^{1 / \gamma}:=K(\varepsilon)
$$

This is equivalent to say that we do not have low order resonances nearby, hence we only have to eliminate higher order ones. When we eliminate the values of $\varepsilon$ for which the Diophantine condition is not fullfiled for some $k$, we only need to worry about controlling 
the measure of the "resonant" sets associated to $|k|_{1} \geq K(\varepsilon)$. From that, and from the exponential in $|k|_{1}$ for the admissible small divisors, we obtain exponentially small estimates for the set of values of $\varepsilon$ for which we can not prove the existence of invariant tori. If $\left.\varepsilon \in] 0, \varepsilon_{0}\right]$ this measre is of order $\exp \left(-1 / \varepsilon_{0}^{c}\right)$, for any $0<c<1 / \gamma$.

Note that we have used $\varepsilon$ because is a natural parameter of the perturbed problem, but this technique can also be applied to other parameters. We will do this in the next section.

\subsection{Other parameters: families of lower dimensional tori}

Let us consider the following truncation of the Hamiltonian (3):

$$
\hat{\omega}^{(0) \top} \hat{I}+\frac{1}{2} z^{\top} \mathcal{B} z+\frac{1}{2} \hat{I}^{\top} \mathcal{C} \hat{I} .
$$

Note that, for this truncation, there exists a $r$ parametric family of $r$-dimensional invariant tori around the initial torus. One can ask what happens to this family when the nonintegrable part (including the quasiperiodic perturbations) is added. The natural parameters in this case are the frequencies $\hat{\omega}$ of the tori of the family. We will work with this parameter as follows: if for every $\hat{\omega}$ we perform the canonical transformation

$$
\hat{I} \rightarrow \hat{I}+\mathcal{C}^{-1}\left(\hat{\omega}-\hat{\omega}^{(0)}\right),
$$

on the Hamiltonian (13), we obtain (skipping the constant term) a Hamiltonian like (13), replacing $\hat{\omega}^{(0)}$ by $\hat{\omega}$. So, we have that $\hat{I}=0, z=0$ is a $r$-dimensional reducible torus but now with vector of basic frequencies given by $\hat{\omega}$. If we consider the Hamiltonian (3), and we perform the transformation (14), it is not difficult to see from conditions P1 and P2 that the $\hat{\omega}$-torus obtained from the truncated normal form remains as an invariant reducible torus for the Hamiltonian (3), plus an error of $O_{2}\left(\hat{\omega}-\hat{\omega}^{(0)}\right)$. Then, the idea is to consider $\hat{\omega}$ as a new perturbative parameter (in fact the small parameter is $\hat{\omega}-\hat{\omega}^{(0)}$ ). In this case we can apply the same technique as in section 2.4 to control the measure of the destroyed tori. It turns out that this measure is exponentially small with the distance to the initial torus.

In fact, the proof has been done working simultaneously with both parameters $\varepsilon$ and $\hat{\omega}$. This allows to derive all the results mentioned before in an unified way. To work with those parametrizations, we ask for smooth dependence of the Hamiltonian with respect to the parameters. Note that at every step of the inductive process we need to remove the set of resonant values of the parameters (that are dense), and this does not allow to keep, in principle, any kind of smooth dependence for the Hamiltonian with respect to the parameters (because now the parameters move on a set with empty interior). Fortunately, there are many solutions to this problem. One possibility is to work, at every step of the inductive procedure, with a finite number of terms in the different Fourier expansion with respect to $\theta$. Hence, we can use that the remainder of the truncated expressions is exponentially small with the order of truncation and then, if we choose a suitable increasing sequence of orders for the truncation at the different steps (going to infinity with the index of the step), we can show the convergence of the sequence of changes given in section 2.3 (with equations $\left(e q_{1}\right)-\left(e q_{5}\right)$ truncated at the corresponding order) on a suitable set of parameters. Then, since we only need to deal with a finite number of resonances at every step, we can work on open sets with respect to the different parameters, and to keep the 
smooth parametric dependences in those sets. This smooth dependence allows to bound the measure of the resonances in the sets of parameters. Those ideas are for example used in [1], [6] or [19]. Another possibility is to replace the (smooth) initial parametric dependence with a Lipschitz one (this has already been done in [21] or [23]). We can see that it is possible to keep this Lipschitz dependence at every step (with analogous techniques as the ones used to control a $C^{1}$ dependence), and this kind of dependence suffices to bound the measure of the resonant sets. In this paper we have chosen this Lipschitz formulation, that we describe more precisely in section 5.1.1.

\section{Statement of the results}

We use here the notations of the previous sections to give in a more concrete form the results that we have described along the paper. They can be formulated in the following form:

Theorem 1 Let us consider a Hamiltonian of the form (4), satisfying the following hypothesis:

(i) $H_{*}$ and $\hat{\mathcal{H}}$ are analytic with respect to $(\theta, x, \hat{I}, y)$ around $z=0$ and $\hat{I}=0$, with $2 \pi$-periodic dependence on $\theta$, for any $\varepsilon \in \mathcal{I}_{0}:=\left[0, \varepsilon_{0}\right]$, in a domain that is independent on $\varepsilon$. The dependence on $\varepsilon$ is assumed to be $C^{2}$, and the derivatives of the Hamiltonian $\hat{\mathcal{H}}$ with respect to $\varepsilon$ are also analytic in $(\theta, x, \hat{I}, y)$ on the same domain.

(ii) $\mathcal{B}$ is a symmetric constant matrix such that $J_{m} \mathcal{B}$ is diagonal with different eigenvalues $\lambda^{\top}=\left(\lambda_{1}, \ldots, \lambda_{m},-\lambda_{1}, \ldots,-\lambda_{m}\right)$.

(iii) $\mathcal{C}$ is a symmetric constant matrix with $\operatorname{det} \mathcal{C} \neq 0$.

(iv) For certain $\mu_{0}>0$ and $\gamma>r+s-1$, the following Diophantine conditions hold:

$$
\left|i k^{\top} \omega^{(0)}+l^{\top} \lambda\right| \geq \frac{\mu_{0}}{|k|_{1}^{\gamma}}, \quad k \in \mathbb{Z}^{r+s} \backslash\{0\}, \quad l \in \mathbb{N}^{2 m}, \quad|l|_{1} \leq 2 .
$$

Then, under certain generic nondegeneracy conditions for the Hamiltonian $H$ (that are given explicitly in NDC at the end of section 5.4.2), the following assertions hold:

(a) There exists a Cantor set $\mathcal{I}_{*} \subset \mathcal{I}_{0}$, such that for every $\varepsilon \in \mathcal{I}_{*}$ the Hamiltonian $H$ has a reducible $(r+s)$-dimensional invariant torus with vector of basic frequencies $\omega^{(0)}$. Moreover, for every $0<\sigma<1$ :

$$
\operatorname{mes}\left([0, \bar{\varepsilon}] \backslash \overline{\mathcal{I}}_{*}\right) \leq \exp \left(-(1 / \bar{\varepsilon})^{\frac{\sigma}{\gamma}}\right),
$$

if $\bar{\varepsilon}$ is small enough (depending on $\sigma$ ) where, for every $\bar{\varepsilon}, \overline{\mathcal{I}}_{*}=\overline{\mathcal{I}}_{*}(\bar{\varepsilon}):=[0, \bar{\varepsilon}] \cap \mathcal{I}_{*}$.

(b) Given $R_{0}>0$ small enough and a fixed $0 \leq \varepsilon \leq R_{0}^{\frac{\gamma}{\gamma+1}}$, there exists a Cantor set $\mathcal{W}_{*}\left(\varepsilon, R_{0}\right) \subset\left\{\hat{\omega} \in \mathbb{R}^{r}:\left|\hat{\omega}-\hat{\omega}^{(0)}\right| \leq R_{0}\right\}:=\mathcal{V}\left(R_{0}\right)$, such that for every $\hat{\omega} \in \mathcal{W}_{*}\left(\varepsilon, R_{0}\right)$ the Hamiltonian $H$ corresponding to this fixed value of $\varepsilon$, has a reducible $(r+s)$ dimensional invariant torus with vector of basic frequencies $\omega, \omega^{\top}=\left(\hat{\omega}^{\top}, \tilde{\omega}^{(0) \top}\right)$. Moreover, for every $0<\sigma<1$, if $R_{0}$ is small enough (depending on $\sigma$ ):

$$
\operatorname{mes}\left(\mathcal{V}\left(R_{0}\right) \backslash \mathcal{W}_{*}\left(\varepsilon, R_{0}\right)\right) \leq \exp \left(-\left(1 / R_{0}\right)^{\frac{\sigma}{\gamma+1}}\right) .
$$

Here, mes $(A)$ denotes the Lebesgue measure of the set $A$. 


\subsection{Remarks}

The result $(b)$ has special interest if we take $\varepsilon=0$. It shows that for the unperturbed system, around the initial $r$-dimensional reducible torus there exist an $r$-dimensional family (with Cantor structure) of $r$-dimensional reducible tori parametrized by $\hat{\omega} \in \mathcal{W}_{*}\left(0, R_{0}\right)$, with relative measure for the complementary of the Cantor set exponentially small with $R_{0}$, for values of $\hat{\omega} R_{0}$-close to $\hat{\omega}^{(0)}$. There are previous results on the existence of these lower dimensional tori (see the references), but the estimates on the measure of preserved tori close to a given one are not so good as the ones presented here.

Moreover, we have the same result around every $(r+s)$-dimensional torus that we can obtain for the perturbed system for some $\varepsilon \neq 0$ small enough, if we assume that their intrinsic and normal frequencies verify the same kind of Diophantine bounds as the frequencies of the unperturbed torus. In this case, for every $R_{0}$ small enough we have a (Cantor) family of $(r+s)$-dimensional reducible tori parametrized by $\hat{\omega} \in \mathcal{W}_{*}\left(\varepsilon, R_{0}\right)$, with the same kind of exponentially small measure with respect to $R_{0}$ on the complementary of this set. To prove it, we remark that we can reduce to the case $\varepsilon=0$ if we note that it is easy to see that Theorem 1 also holds if the unperturbed Hamiltonian depends on $\theta$ and not only on $\hat{\theta}$ (that is, if the initial torus is $(r+s)$-dimensional).

If the initial torus is normally hyperbolic, the problem is easier. For instance, it is possible to prove the existence of invariant tori without using reducibility conditions. Then, in case $(a)$, one obtains an open set of values of $\varepsilon$ for which the torus exists, although its normal flow could not be reducible. The reason is that the intrinsic frequencies of the torus are fixed with respect to $\varepsilon$ and the normal eigenvalues (that depend on $\varepsilon$ ) do not produce extra small divisors if we consider only equations $\left(e q_{1}\right)-\left(e q_{3}\right)$ of the iterative scheme described in section 2.3 (we take $G=0$ and $F=0$ in equation (7)). Note that now we can solve $\left(e q_{2}\right)$ using a fixed point method, because the matrices $J_{m} \mathcal{B}^{(n)}$ are $\varepsilon$ close to the initial hyperbolic matrix $J_{m} \mathcal{B}$ (that is supposed to be reducible). This makes unnecessary to consider $\left(e q_{4}\right)$ and $\left(e q_{5}\right)$. Of course, the tori produced in this way are not necessarily reducible. If one wants to ensure reducibility, it is necessary to use the normal eigenvalues and this can produce (depending on some conditions on those eigenvalues, see [23]) a Cantor set of $\varepsilon$ of the same measure as the one in $(a)$. If we consider the case $(b)$ when the normal behaviour is hyperbolic, the results do not change with respect to the normally elliptic case. As we are "moving" the intrinsic frequencies, we have to take out the corresponding resonances. The order (when $R_{0}$ goes to zero) of the measure of these resonances is still exponentially small with $R_{0}$ (see lemma 16).

Finally, let us recall that the Diophantine condition $(i v)$ is satisfied for all the frequen$\operatorname{cies} \omega^{(0)}$ and eigenvalues $\lambda$, except for a set of zero measure.

\section{Applications}

In this section we are going to illustrate the possible applications of these results to some concrete problems of celestial mechanics.

\subsection{The bicircular model near $L_{4,5}$}

The bicircular problem is a first approximation to study the motion of a small particle in the Earth-Moon system, including perturbations coming for the Sun. In this model it is 
assumed that Earth and Moon revolve in a circular orbits around their centre of masses, and that this centre of masses moves in circular orbit around the Sun. Usually, in order to simplify the equations, the units of lenght, time and mass are chosen such that the angular velocity of rotation of Earth and Moon (around their centre of masses), the sum of masses of Earth and Moon and the gravitational constant are all equal to one. With these normalized units, the Earth-Moon distance is also one. The system of reference is defined as follows: the origin is taken at the centre of mass of the Earth-Moon system, the $X$ axis is given by the line that goes from Moon to Earth, the $Z$ axis has the direction of the angular momentum of Earth and Moon and the $Y$ axis is taken such that the system is orthogonal and positive-oriented. Note that, in this (non-inertial) frame, called synodic system, Earth and Moon have fixed positions and the Sun is rotating around the barycentre of the Earth-Moon system. If we define momenta $P_{X}=\dot{X}-Y, P_{Y}=\dot{Y}+X$ and $P_{Z}=\dot{Z}$, in these coordinates, the motion of a infinitessimal particle moving under the gravitational attraction of Earth, Moon and Sun is given by the Hamiltonian

$$
H=\frac{1}{2}\left(P_{X}^{2}+P_{Y}^{2}+P_{Z}^{2}\right)+Y P_{X}-X P_{Y}-\frac{1-\mu}{r_{P E}}-\frac{\mu}{r_{P M}}-\frac{m_{s}}{r_{P S}}-\frac{m_{s}}{a_{s}^{2}}(Y \sin \theta-X \cos \theta),
$$

where $\theta=w_{S} t$, being $w_{S}$ the mean angular velocity of the Sun in synodic coordinates, $\mu$ the mass parameter for the Earth-Moon system, $a_{s}$ the semimajor axis of the Sun, $m_{s}$ the Sun mass, and $r_{P E}, r_{P M}, r_{P S}$ are defined in the following form:

$$
\begin{aligned}
r_{P E}^{2} & =(X-\mu)^{2}+Y^{2}+Z^{2} \\
r_{P M}^{2} & =(X-\mu+1)^{2}+Y^{2}+Z^{2} \\
r_{P S}^{2} & =\left(X-X_{s}\right)^{2}+\left(Y-Y_{s}\right)^{2}+Z^{2}
\end{aligned}
$$

where $X_{s}=a_{s} \cos \theta$ and $Y_{s}=-a_{s} \sin \theta$.

Note that one can look at this model as a time-periodic perturbation of an autonomous system, the Restricted Three Body Problem (usually called RTBP, see [34] for definition and basic properties). Hence, the Hamiltonian is of the form

$$
H=H_{0}(x, y)+\varepsilon H_{1}(x, y, t),
$$

where $\varepsilon$ is a parameter such that $\varepsilon=0$ corresponds to the unperturbed RTBP and $\varepsilon=1$ to the bicircular model with the actual values for the perturbation.

Note that the bicircular model is not dynamically consistent, because the motion of Earth, Moon and Sun does not follow a true orbit of the system (we are not taking into account the interaction between the Sun and the Earth-Moon system). Nevertheless, numerical simulation shows that, in some regions of the phase space, this model gives the same qualitative behaviour as the real system and this makes it worth to study (see [32]).

We are going to focus in the dynamics near the equilateral points $L_{4,5}$ of the EarthMoon system. These points are linearly stable for the unperturbed problem $(\varepsilon=0)$, so we can associate three families of periodic (Lyapounov) orbits to them: the short period family, the long period family and the vertical family of periodic orbits. Classical results about these families can be found in [34].

When the perturbation is added the points $L_{4,5}$ become (stable) periodic orbits with the same period as the perturbation. These orbits become unstable for the actual value of the perturbation ( $\varepsilon=1$ in the notation above). In this last case, numerical simulation 
shows the existence of a region of stability not very close to the orbit and outside of the plane of motion of Earth and Moon. This region seems to be centered around some of the (Lyapounov) periodic orbits of the vertical family. See [16] or [32] for more details.

Let us consider the dynamics near $L_{4,5}$ for $\varepsilon$ small. In this case, the equilibrium point has been replaced by a small periodic orbit. Our results imply that the three families of Lyapounov periodic orbits become three cantorian families of $2-\mathrm{D}$ invariant tori, adding the perturbing frequency to the one of the periodic orbit. Moreover, the Lyapounov tori (the 2-D invariant tori of the unperturbed problem that are obtained by "product" of two families of periodic orbits) become 3 -D invariant tori, provided they are nonresonant with the perturbation. Finally, the maximal dimension (3-D) invariant tori of the unperturbed problem become 4-D tori, adding the frequency of the Sun to the ones they already had (this last result is already contained in [23]).

Now let us consider $\varepsilon=1$. This value of $\varepsilon$ is too big to apply these results. In particular, $\varepsilon$ is big enough to cause a change of stability in the periodic orbit that replaces the equilibrium point. Hence, if one wants to apply the results of this paper to this case, it is necessary to start by putting the Hamiltonian in a suitable form. To describe the dynamics near the unstable periodic orbit that replaces the equilibrium point, we can perform some steps of a normal form procedure to write the Hamiltonian as an autonomous (and integrable) Hamiltonian plus a small time dependent periodic perturbation (see [16], [22] or [32] for more details about these kind of computations). Then, if we are close enough to the periodic orbit, Theorem 1 applies and we have invariant tori of dimensions 1, 2 and 3. They are in the "central" directions of the periodic orbit.

The application to the stable region that is in the vertical direction is more difficult. A possiblity is to compute (numerically) an approximation to a $2-\mathrm{D}$ invariant torus of the vertical family (note that its existence has not already been proved rigorously) and to perform some steps of a normal form procedure, in order to write the problem as an integrable autonomous Hamiltonian plus a time dependent periodic perturbation. Then, if the (approximate) torus satisfies the equations within a small enough error, it should be possible to show the existence of a torus nearby, and to establish that it is stable and surrounded by invariant tori of dimensions 1 to 4 . Numerical experiments suggest (see [16] or [32]) that this is what happens in this case.

\subsubsection{Extensions}

In fact, the bicircular model is only the first step in the study of the dynamics near the libration points of Earth-Moon system. One can construct better models taking into account the non-circular motion of Earth and Moon (see [8], [14], [16]). Our results can be applied to these models in the same way it has been done in the bicircular case. The main difference is that now the equilibrium point is replaced by a quasiperiodic solution that, due to the resonances, does not exist for all values of $\varepsilon$ but only for a Cantor set of them (see [23]).

\subsection{Halo orbits}

Let us consider the Earth and Sun as a RTBP, and let us focus in the dynamics near the equilibrium point that it is in between (the so called $L_{1}$ point). It is well known the existence of a family of periodic orbits (called Halo orbits, see [29]) such that, when one 
looks at them from the Earth, they seem to describe an halo around the solar disc. These orbits are a very suitable place to put a spacecraft to study the Sun: from that place, the Sun is always visible and it is always possible to send data back to Earth (because the probe does not cross the solar disc, otherwise the noise coming from the Sun would make communications impossible). These orbits have been used by missions ISEE-C (from 1978 to 1982) and SOHO (launched in 1995).

In the RTBP, Halo orbits are a one parameter family of periodic orbits with a normal behaviour of the type centre $\times$ saddle. Unfortunately, the RTBP is too simple to produce good approximations to the dynamics. If one wants to have a cheap station keeping it is necessary to compute the nominal orbit with a very accurate model (see [13], [14], [15] and $[16])$.

The usual analytic models for this problem are written as an autonomous Hamiltonian (the RTBP) plus the effect coming from the real motion of Earth and Moon, the effect of Venus, etc. All these effects can be modelled very accurately using quasiperiodic functions that depend on time in a quasiperiodic way. Hence, we end up with an autonomous Hamiltonian plus a quasiperiodic time dependent perturbation with $r>0$ frequencies. As usual, we add a parameter $\varepsilon$ in front of this perturbation.

Then, Theorem 1 implies that, if $\varepsilon$ is small enough, the Halo orbits become a cantorian family of $(r+1)$-D invariant tori. The normal behaviour of these tori is also of the type centre $\times$ saddle.

To study the case $\varepsilon=1$ we refer to the remarks for the case of the bicircular problem.

\section{Proofs}

This section contains the proof of Theorem 1 . It has been split in several parts to simplify the reading. Section 5.1 introduces the basic notation used along the proof. In section 5.2 we give the basic lemmas needed during the proof. Section 5.3 gives quantitative estimates on one step of the iterative scheme and section 5.4 contains the technical details of the proof.

\section{$5.1 \quad$ Notations}

Here we introduce some of the notations used to prove the different results.

\subsubsection{Norms and Lipschitz constants}

As usual we denote by $|v|$ the absolute value of $v \in \mathbb{C}$, and we use the same notation to refer to the (maximum) vectorial or matrix norm on $\mathbb{C}^{n}$ or $\mathbb{M}_{n_{1}, n_{2}}(\mathbb{C})$.

Let us denote by $f$ an analytic function defined on a complex strip of width $\rho>0$, having $r$ arguments and being $2 \pi$-periodic in all of them. The range of this function can be in $\mathbb{C}, \mathbb{C}^{n}$ or $\mathbb{M}_{n_{1}, n_{2}}(\mathbb{C})$. If we write its Fourier expansion as

$$
f(\theta)=\sum_{k \in \mathbb{Z}^{r}} f_{k} \exp \left(i k^{\top} \theta\right)
$$

we can introduce the norm

$$
|f|_{\rho}=\sum_{k \in \mathbb{Z}^{r}}\left|f_{k}\right| \exp \left(|k|_{1} \rho\right)
$$


Let $f(\theta, q)$ be a $2 \pi$-periodic function on $\theta$, and analytic on the domain

$$
\mathcal{U}_{\rho, R}^{r, m}=\left\{(\theta, q) \in \mathbb{C}^{r} \times \mathbb{C}^{m}:|\operatorname{Im} \theta| \leq \rho,|q| \leq R\right\}
$$

If we write its Taylor expansion around $q=0$ as:

$$
f(\theta, q)=\sum_{l \in \mathbb{N}^{m}} f_{l}(\theta) q^{l}
$$

then, from this expansion we define the norm:

$$
|f|_{\rho, R}=\sum_{l \in \mathbb{N}^{m}}\left|f_{l}\right|_{\rho} R^{|l|_{1}}
$$

If $f$ takes values in $\mathbb{C}$, we put $\nabla f$ to denote the gradient of $f$ with respect to $(\theta, q)$.

Now, we introduce the kind of Lipschitz dependence considered. Assume that $f(\varphi)$ is a function defined for $\varphi \in \mathcal{E}, \mathcal{E} \subset \mathbb{R}^{j}$ for some $j$, and with values in $\mathbb{C}, \mathbb{C}^{n}$ or $\mathbb{M}_{n_{1}, n_{2}}(\mathbb{C})$. We call $f$ a Lipschitz function with respect to $\varphi$ on the set $\mathcal{E}$ if:

$$
\mathcal{L}_{\mathcal{E}}\{f\}=\sup _{\substack{\varphi_{1}, \varphi_{2} \in \mathcal{E} \\ \varphi_{1} \neq \varphi_{2}}} \frac{\left|f\left(\varphi_{2}\right)-f\left(\varphi_{1}\right)\right|}{\left|\varphi_{2}-\varphi_{1}\right|}<+\infty
$$

The value $\mathcal{L}_{\mathcal{E}}\{f\}$ is called the Lipschitz constant of $f$ on $\mathcal{E}$. For these kind of functions we define $\|f\|_{\mathcal{E}}=\sup _{\varphi \in \mathcal{E}}|f(\varphi)|$.

Similarly, if $f(\theta, \varphi)$ is a $2 \pi$-periodic analytic function on $\theta$ for every $\varphi \in \mathcal{E}$, we denote:

$$
\mathcal{L}_{\mathcal{E}, \rho}\{f\}=\sup _{\substack{\varphi_{1}, \varphi_{2} \in \mathcal{E} \\ \varphi_{1} \neq \varphi_{2}}} \frac{\left|f\left(., \varphi_{2}\right)-f\left(., \varphi_{1}\right)\right|_{\rho}}{\left|\varphi_{2}-\varphi_{1}\right|}
$$

In the same way we can introduce $\mathcal{L}_{\mathcal{E}, \rho, R}\{f\}$, if we work with $f(\theta, q, \varphi)$ and the norm $|\cdot|_{\rho, R}$. We can also extend $\|\cdot\|_{\mathcal{E}}$ to both cases to define $\|\cdot\|_{\mathcal{E}, \rho}$ and $\|\cdot\|_{\mathcal{E}, \rho, R}$.

\subsubsection{Canonical transformations}

The changes of variables are performed by means of a Lie series method, with a suitable generating function. For the sake of clarity, we will use here the same notations for the different variables as in the formulation of the results. We want to keep the quasiperiodic time dependence (after each transformation) with the same vector of basic frequencies $\tilde{\omega}^{(0)}$ as the initial one. This is achieved when the generating function does not depend on $\tilde{I}$.

Let us consider a generating function $S(\theta, x, \hat{I}, y)$ such that $\nabla S$ depends analytically on $(\theta, x, \hat{I}, y)$ and it is $2 \pi$-periodic in $\theta$. The equations related to the Hamiltonian function $S$ are

$$
\dot{\hat{\theta}}=\left(\frac{\partial S}{\partial \hat{I}}\right)^{\top}, \dot{\tilde{\theta}}=\left(\frac{\partial S}{\partial \tilde{I}}\right)^{\top}=0, \dot{\hat{I}}=-\left(\frac{\partial S}{\partial \hat{\theta}}\right)^{\top}, \dot{\tilde{I}}=-\left(\frac{\partial S}{\partial \hat{\theta}}\right)^{\top}, \dot{z}=J_{m}\left(\frac{\partial S}{\partial z}\right)^{\top} .
$$

We denote by $\Psi_{t}^{S}(\theta, x, I, y)$ the flow at time $t$ of $S$ with initial conditions $(\theta, x, I, y)$ when $t=0$. We note that $\Psi_{t}^{S}$ is (for a fixed $\mathrm{t}$ ) a canonical change of variables that acts in 
a trivial way on $\tilde{\theta}$. If we put $(\theta(t), x(t), I(t), y(t))=\Psi_{t}^{S}(\theta(0), x(0), I(0), y(0))$, we can express the change as:

$$
\begin{gathered}
\hat{\theta}(t)=\hat{\theta}(0)+\int_{0}^{t}\left(\frac{\partial S}{\partial \hat{I}}(\theta(\tau), x(\tau), \hat{I}(\tau), y(\tau))\right)^{\top} d \tau \\
I(t)=I(0)-\int_{0}^{t}\left(\frac{\partial S}{\partial \theta}(\theta(\tau), x(\tau), \hat{I}(\tau), y(\tau))\right)^{\top} d \tau, \\
z(t)=z(0)+J_{m} \int_{0}^{t}\left(\frac{\partial S}{\partial z}(\theta(\tau), x(\tau), \hat{I}(\tau), y(\tau))\right)^{\top} d \tau,
\end{gathered}
$$

and $\tilde{\theta}(t)=\tilde{\theta}(0)$. We note that the function $\Psi_{t}^{S}-I d$ does not depend on the auxiliar variables $\tilde{I}$. Then, we put $\theta(0)=\theta, \hat{I}(0)=\hat{I}$ and $z(0)=z$ to introduce the transformations $\hat{\Psi}_{t}^{S}$ and $\hat{\Phi}_{t}^{S}$, defined as $\hat{\Psi}_{t}^{S}(\theta, x, \hat{I}, y)=(\theta(t), x(t), \hat{I}(t), y(t))$ and $\hat{\Phi}_{t}^{S}=\hat{\Psi}_{t}^{S}-I d$. It is not difficult to check that $\hat{\Phi}_{t}^{S}(\theta, x, \hat{I}, y)$ is (for a fixed $t$ ) $2 \pi$-periodic in $\theta$.

If we consider the Hamiltonian function $H$ of $(6)$, and we put

$$
H^{* *}=\left\{H^{*}, S\right\}-\frac{\partial S}{\partial \tilde{\theta}} \tilde{\omega}^{(0)},
$$

$\Psi_{t}^{S}$ transforms the Hamiltonian $H$ into

$$
H \circ \Psi_{t}^{S}(\theta, x, I, y)=\tilde{\omega}^{(0) \top} \tilde{I}+H^{*}(\theta, x, \hat{I}, y)+t H^{* *}(\theta, x, \hat{I}, y)+\Sigma_{t}\left(H^{* *}, S\right)(\theta, x, \hat{I}, y),
$$

where

$$
\Sigma_{t}\left(H^{* *}, S\right)=\sum_{j \geq 2} \frac{t^{j}}{j !} L_{S}^{j-1}\left(H^{* *}\right),
$$

with $L_{S}^{0}\left(H^{* *}\right)=H^{* *}$ and $L_{S}^{j}\left(H^{* *}\right)=\left\{L_{S}^{j-1}\left(H^{* *}\right), S\right\}$, for $j \geq 1$.

We remark that if we transform a Hamiltonian function $H$ by the canonical change of variables $\Psi_{t}^{S}$, we only need to control the transformation $\hat{\Phi}_{t}^{S}$ and to see that the new Hamiltonian, $H \circ \Psi_{t}^{S}$, is well defined on a suitable domain.

Finally, as the change of variables is selected as the flow at time one of a Hamiltonian $S$, in what follows we will omit the subscript $t$ and we will assume that it means $t=1$.

\subsection{Basic lemmas}

\subsubsection{Lemmas on norms and Lipschitz constants}

In this section we give some bounds used when working with the norms and Lipschitz constants introduced in section 5.1.1. We follow here the same notations of section 5.1.1 for the different analytic functions used in the lemmas.

Lemma 1 Let $f(\theta)$ and $g(\theta)$ be analytic functions on a strip of width $\rho>0,2 \pi$-periodic in $\theta$ and taking values in $\mathbb{C}$. Let us denote by $f_{k}$ the Fourier coefficients of $f, f(\theta)=$ $\sum_{k \in \mathbb{Z}^{r}} f_{k} \exp \left(i k^{\top} \theta\right)$. Then we have:

(i) $\left|f_{k}\right| \leq|f|_{\rho} \exp \left(-|k|_{1} \rho\right)$.

(ii) $|f g|_{\rho} \leq|f|_{\rho}|g|_{\rho}$. 
(iii) For every $0<\rho_{0}<\rho$

$$
\left|\frac{\partial f}{\partial \theta_{j}}\right|_{\rho-\rho_{0}} \leq \frac{|f|_{\rho}}{\rho_{0} \exp (1)}, j=1, \ldots, r .
$$

(iv) Let $\left\{d_{k}\right\}_{k \in \mathbb{Z}^{r} \backslash\{0\}} \subset \mathbb{C}$, with the following bounds:

$$
\left|d_{k}\right| \geq \frac{\mu}{|k|_{1}^{\gamma}} \exp \left(-\delta|k|_{1}\right)
$$

for some $\mu>0, \gamma \geq 0,0 \leq \delta<\rho$. If we assume that $\bar{f}=0$, then the function $g$ defined as

$$
g(\theta)=\sum_{k \in \mathbb{Z}^{r} \backslash\{0\}} \frac{f_{k}}{d_{k}} \exp \left(i k^{\top} \theta\right),
$$

satisfies the bound

$$
|g|_{\rho-\rho_{0}} \leq\left(\frac{\gamma}{\left(\rho_{0}-\delta\right) \exp (1)}\right)^{\gamma} \frac{|f|_{\rho}}{\mu}
$$

for every $\left.\rho_{0} \in\right] \delta, \rho[$.

All these bounds can be extended to the case when $f$ and $g$ take values in $\mathbb{C}^{n}$ or $\mathbb{M}_{n_{1}, n_{2}}(\mathbb{C})$. Of course, in the matrix case, in (ii) it is necessary that the product $f g$ be well defined.

Proof: Items (i) and (ii) are easily verified. Proofs of (iii) and (iv) are essentially contained in [23], but working with the supremum norm.

Lemma 2 Let $f(\theta, q)$ and $g(\theta, q)$ be analytic functions on a domain $\mathcal{U}_{\rho, R}^{r, m}$ and $2 \pi$-periodic in $\theta$. Then we have:

(i) If we expand $f(\theta, q)=\sum_{l \in \mathbb{N}^{m}} f_{l}(\theta) q^{l}$, then $\left|f_{l}\right|_{\rho} \leq \frac{|f|_{\rho, R}}{R^{l} \|_{1}}$.

(ii) $|f g|_{\rho, R} \leq|f|_{\rho, R}|g|_{\rho, R}$.

(iii) For every $0<\rho_{0}<\rho$ and $0<R_{0}<R$, we have:

$$
\left|\frac{\partial f}{\partial \theta_{j}}\right|_{\rho-\rho_{0}, R} \leq \frac{|f|_{\rho, R}}{\rho_{0} \exp (1)}, j=1, \ldots, r
$$

and

$$
\left|\frac{\partial f}{\partial q_{j}}\right|_{\rho, R-R_{0}} \leq \frac{|f|_{\rho, R}}{R_{0}}, j=1, \ldots, m .
$$

As in lemma 1 , all the bounds hold if $f$ and $g$ take values in $\mathbb{C}^{n}$ or $\mathbb{M}_{n_{1}, n_{2}}(\mathbb{C})$.

Proof: Items (i) and (ii) are straightforward. The first part of (iii) is a consequence of lemma 1 . The second part is obtained applying standard Cauchy estimates to the function $F(q)=\sum_{l \in \mathbb{N}^{m}}\left|f_{l}\right|_{\rho} q^{l}$. 
Lemma 3 Let us take $0<\rho_{0}<\rho$ and $0<R_{0}<R$, and let us consider analytic functions $\Theta(\theta, q)$ (with values in $\mathbb{C}^{r}$ ) and $X(\theta, q)$ (with values in $\mathbb{C}^{m}$ ), both $2 \pi$-periodic on $\theta$, and such that $|\Theta|_{\rho_{0}, R_{0}} \leq \rho-\rho_{0}$ and $|X|_{\rho_{0}, R_{0}} \leq R-R_{0}$. Let $f(\theta, q)$ be a given (2 $\pi$-periodic on $\theta)$ analytic function. If we define:

$$
F(\theta, q)=f(\theta+\Theta(\theta, q), q+X(\theta, q))
$$

then, $|F|_{\rho_{0}, R_{0}} \leq|f|_{\rho, R}$.

Proof: Expanding $f$ in Taylor series (as $(i)$ in lemma 2) one obtains the expansion of $F$ as a function of $\Theta$ and $X$. Then the bound is a consequence of (ii) in 2 .

Lemma 4 Let us consider $\Theta^{(j)}$ and $X^{(j)}, j=1,2$, with the same conditions as $\Theta$ and $X$ lemma 3 , but with the following bounds: $\left|\Theta^{(j)}\right|_{\rho_{0}, R_{0}} \leq \rho-\rho_{0}-\delta$ and $\left|X^{(j)}\right|_{\rho_{0}, R_{0}} \leq R-R_{0}-\chi$, with $0<\delta<\rho-\rho_{0}$ and $0<\chi<R-R_{0}$. Then, if we define

$$
F^{(j)}(\theta, q)=f\left(\theta+\Theta^{(j)}(\theta, q), q+X^{(j)}(\theta, q)\right), j=1,2,
$$

one has

$$
\left|F^{(1)}-F^{(2)}\right|_{\rho_{0}, R_{0}} \leq\left(\frac{\left|\Theta^{(1)}-\Theta^{(2)}\right|_{\rho_{0}, R_{0}}}{\exp (1) \delta}+m \frac{\left|X^{(1)}-X^{(2)}\right|_{\rho_{0}, R_{0}}}{\chi}\right)|f|_{\rho, R} .
$$

Proof: We can use here the same ideas as in lemma 3, combined with the ones used to prove lemmas 1 and 2.

Now we give some basic results related to the Lipschitz dependences introduced in section 5.1.1. For that purpose, we work with a parameter $\varphi$ on the set $\mathcal{E} \subset \mathbb{R}^{j}$, for some $j \geq 1$.

Lemma 5 We consider Lipschitz functions $f(\varphi)$ and $g(\varphi)$ defined for $\varphi \in \mathcal{E}$ with values in $\mathbb{C}$, then:

(i) $\mathcal{L}_{\mathcal{E}}\{f+g\} \leq \mathcal{L}_{\mathcal{E}}\{f\}+\mathcal{L}_{\mathcal{E}}\{g\}$.

(ii) $\mathcal{L}_{\mathcal{E}}\{f g\} \leq\|f\|_{\mathcal{E}} \mathcal{L}_{\mathcal{E}}\{g\}+\|g\|_{\mathcal{E}} \mathcal{L}_{\mathcal{E}}\{f\}$.

(iii) $\mathcal{L}_{\mathcal{E}}\{f / g\} \leq\left\|\frac{1}{g}\right\|_{\mathcal{E}} \mathcal{L}_{\mathcal{E}}\{f\}+\|f\|_{\mathcal{E}}\left\|_{g} \frac{1}{g}\right\|_{\mathcal{E}}^{2} \mathcal{L}_{\mathcal{E}}\{g\}$, if $g$ does not vanish.

Moreover, $(i)$ holds if $f$ and $g$ take values in $\mathbb{C}^{n}$ or $\mathbb{M}_{n_{1}, n_{2}}(\mathbb{C})$, and (ii) also holds when $f$ and $g$ are matrix-valued functions (such that the matrix product $f g$ is well defined).

Proof: It is straightforward.

Lemma 6 We assume that $B(\varphi)$ is defined for $\varphi \in \mathcal{E}$ with values in $\mathbb{M}_{n, n}(\mathbb{C})$, and that $B^{-1}$ exist for all $\varphi$. Then

$$
\mathcal{L}_{\mathcal{E}}\left\{B^{-1}\right\} \leq\left\|B^{-1}\right\|_{\mathcal{E}}^{2} \mathcal{L}_{\mathcal{E}}\{B\}
$$

Proof: It is straightforward.

Remark 1 In lemmas 5 and 6 , we obtain analogous results if we work with functions of the form $f(\theta, \varphi)$ or $f(\theta, q, \varphi)$, defined for $\varphi \in \mathcal{E}$ and analytical with respect to the variables $(\theta, q)$ and the norms $|.|_{\rho},|\cdot|_{\rho, R}$. 
Lemma 7 We assume that $f(\theta, \varphi)$ is, for every $\varphi \in \mathcal{E}$, an analytic $2 \pi$-periodic function in $\theta$ on a strip of width $\rho>0$, with Lipschitz dependence with respect to $\varphi$. Let us expand $f(\theta, \varphi)=\sum_{k \in \mathbb{Z}^{r}} f_{k}(\varphi) \exp \left(i k^{\top} \theta\right)$. Then, we have:

(i) $\mathcal{L}_{\mathcal{E}}\left\{f_{k}\right\} \leq \mathcal{L}_{\mathcal{E}, \rho}\{f\} \exp \left(-|k|_{1} \rho\right)$.

(ii) For every $0<\rho_{0}<\rho$

$$
\mathcal{L}_{\mathcal{E}, \rho-\rho_{0}}\left\{\frac{\partial f}{\partial \theta_{j}}\right\} \leq \frac{\mathcal{L}_{\mathcal{E}, \rho}\{f\}}{\rho_{0} \exp (1)}, j=1, \ldots, r .
$$

(iii) Let $\left\{d_{k}(\varphi)\right\}_{k \in \mathbb{Z}^{r} \backslash\{0\}}$ be a set of complex-valued functions defined for $\varphi \in \mathcal{E}$, with the following bounds:

$$
\left|d_{k}(\varphi)\right| \geq \frac{\mu}{|k|_{1}^{\gamma}} \exp \left(-\delta|k|_{1}\right)
$$

and

$$
\mathcal{L}_{\mathcal{E}}\left\{d_{k}\right\} \leq A+B|k|_{1},
$$

for some $\mu>0, \gamma \geq 0,0 \leq 2 \delta<\rho, A \geq 0$ and $B \geq 0$. As in lemma 1 we assume $\bar{f}=0$ for every $\varphi \in \mathcal{E}$. If

$$
g(\theta, \varphi)=\sum_{k \in \mathbb{Z}^{r} \backslash\{0\}} \frac{f_{k}(\varphi)}{d_{k}(\varphi)} \exp \left(i k^{\top} \theta\right),
$$

then, for every $\rho_{0}, 2 \delta<\rho_{0}<\rho$, we have:

$$
\begin{aligned}
\mathcal{L}_{\mathcal{E}, \rho-\delta_{0}}\{g\} \leq & \left(\frac{\gamma}{\left(\delta_{0}-\delta\right) \exp (1)}\right)^{\gamma} \frac{\mathcal{L}_{\mathcal{E}, \rho}\{f\}}{\mu}+\left(\frac{2 \gamma+1}{\left(\delta_{0}-2 \delta\right) \exp (1)}\right)^{2 \gamma+1} \frac{\|f\|_{\mathcal{E}, \rho}}{\mu^{2}} B+ \\
& +\left(\frac{2 \gamma}{\left(\delta_{0}-2 \delta\right) \exp (1)}\right)^{2 \gamma} \frac{\|f\|_{\mathcal{E}, \rho}}{\mu^{2}} A .
\end{aligned}
$$

Proof: It is analogous to lemma 1, using also the results of lemma 5.

Lemma 8 We assume that $f(\theta, q, \varphi)$ is, for every $\varphi \in \mathcal{E}$, an analytic function on $\mathcal{U}_{\rho, R}^{r, m}$ and $2 \pi$-periodic in $\theta$. Then we have:

(i) If we write $f(\theta, q, \varphi)=\sum_{l \in \mathbb{N}^{m}} f_{l}(\theta, \varphi) q^{l}$, then $\mathcal{L}_{\mathcal{E}, \rho}\left\{f_{l}\right\} \leq \frac{\mathcal{L}_{\mathcal{E}, \rho, R}\{f\}}{R^{\mid l l_{1}}}$.

(ii) For every $0<\rho_{0}<\rho$ and $0<R_{0}<R$, we have:

$$
\mathcal{L}_{\mathcal{E}, \rho-\rho_{0}, R}\left\{\frac{\partial f}{\partial \theta_{j}}\right\} \leq \frac{\mathcal{L}_{\mathcal{E}, \rho, R}\{f\}}{\rho_{0} \exp (1)}, j=1, \ldots, r,
$$

and

$$
\mathcal{L}_{\mathcal{E}, \rho, R-R_{0}}\left\{\frac{\partial f}{\partial q_{j}}\right\} \leq \frac{\mathcal{L}_{\mathcal{E}, \rho, R}\{f\}}{R_{0}}, j=1, \ldots, m
$$

Proof: As in lemma 7, but using now the same ideas as in lemma 2. 


\subsubsection{Lemmas on canonical transformations}

We give here some lemmas that we will use to work with the canonical transformations that we have introduced in section 5.1.2. The purpose is to bound the changes as well as the transformed Hamiltonian. We also take into account the possibility that the generating function depends on a parameter $\varphi \in \mathcal{E}$ in a Lipschitz way.

To simplify the notations in the lemmas of this section, we define

$$
\Delta_{\rho_{0}, R_{0}}=\frac{r}{\rho_{0} \exp (1)}+\frac{r+2 m}{R_{0}}
$$

and we will use (without explicit mention) the notations introduced in section 5.1.

The proofs of lemmas $9,10,11$ and 12 can be obtained from the bounds of lemmas of section 5.2.1. The proof of lemma 10 is essentially contained in [6]. The proof of 12 is similar. The proof of lemma 13 can also be found in [6], where it is proved working with the supremum norm. In our case the proof is analogous from the explicit expressions for the transformation $\hat{\Psi}^{S}$ given in section 5.1.2, using the result of lemma 3 to bound the compositions.

Lemma 9 Let us consider $f(\theta, x, \hat{I}, y)$ and $g(\theta, x, \hat{I}, y)$ complex-valued functions such that $f$ and $\nabla g$ are analytic functions defined on $\mathcal{U}_{\rho, R}^{r+s, r+2 m}, 2 \pi$-periodic on $\theta$. Then, for every $0<\rho_{0}<\rho$ and $0<R_{0}<R$, we have:

$$
|\{f, g\}|_{\rho-\rho_{0}, R-R_{0}} \leq \Delta_{\rho_{0}, R_{0}}|\nabla g|_{\rho, R}|f|_{\rho, R} .
$$

Lemma 10 With the same hypothesis of lemma 9 we have, for the expression $\Sigma(f, g)$ introduced in (16),

$$
|\Sigma(f, g)|_{\rho-\rho_{0}, R-R_{0}} \leq \sum_{j \geq 1} \frac{1}{j+1}\left(\Delta_{\rho_{0}, R_{0}} \exp (1)|\nabla g|_{\rho, R}\right)^{j}|f|_{\rho, R}
$$

Lemma 11 Assume that the complex-valued functions $f(\theta, x, \hat{I}, y, \varphi)$ and $g(\theta, x, \hat{I}, y, \varphi)$ verify that, for every $\varphi \in \mathcal{E}, f$ and $\nabla g$ are analytic functions on $\mathcal{U}_{\rho, R}^{r+s, r+2 m}, 2 \pi$-periodic on $\theta$, with Lipschitz dependence on $\varphi$. Then, if $\|f\|_{\mathcal{E}, \rho, R} \leq F_{1},\|\nabla g\|_{\mathcal{E}, \rho, R} \leq F_{2}, \mathcal{L}_{\mathcal{E}, \rho, R}\{f\} \leq$ $L_{1}$ and $\mathcal{L}_{\mathcal{E}, \rho, R}\{\nabla g\} \leq L_{2}$, we have that, for every $0<\rho_{0}<\rho$ and $0<R_{0}<R$,

$$
\mathcal{L}_{\mathcal{E}, \rho-\rho_{0}, R-R_{0}}\{\{f, g\}\} \leq \Delta_{\rho_{0}, R_{0}}\left(F_{1} L_{2}+F_{2} L_{1}\right) .
$$

Lemma 12 With the same hypothesis of lemma 11, we have:

$$
\mathcal{L}_{\mathcal{E}, \rho-\rho_{0}, R-R_{0}}\{\Sigma(f, g)\} \leq \sum_{j \geq 1}\left(\frac{1}{j+1}\left(\Delta_{\rho_{0}, R_{0}} \exp (1)\right)^{j} F_{2}^{j-1}\left(j L_{2} F_{1}+L_{1} F_{2}\right)\right) .
$$

Lemma 13 We assume that the generating function $S(\theta, x, \hat{I}, y)$ of section 5.1.2 verifies that $\nabla S$ is analytic on $\mathcal{U}_{\rho, R}^{r+s, r+2 m}, 2 \pi$-periodic in $\theta$, with $|\nabla S|_{\rho, R} \leq \delta$, where $\delta<$ $\min \{\rho, R\}$. Then, with the notations of section 5.1.2, we have:

(i) $\left|\hat{\Phi}^{S}\right|_{\rho-\delta, R-\delta} \leq|\nabla S|_{\rho, R}$.

(ii) $\hat{\Psi}^{S}: \mathcal{U}_{\rho-\delta, R-\delta}^{r+s, 2 m+r} \longrightarrow \mathcal{U}_{\rho, R}^{r+s, 2 m+r}$. 


\subsubsection{Convergence lemma}

We will use the following lemma during the proof of Theorem 1, to relate the bounds on the Hamiltonian after $n$ steps of the iterative scheme as a function of bounds for the initial Hamiltonian.

Lemma 14 Let $\left\{K_{n}\right\}_{n \geq 1}$ be a sequence of positive numbers with $K_{n+1} \leq a n^{b} K_{n}^{2} \exp \left(\varrho^{n} c\right)$ if $n \geq 1$, being $a>0, b \geq 0, c>0$ and $1<\varrho<2$. Then:

$$
K_{n+1} \leq \frac{1}{a}\left(\left(\frac{5}{3}\right)^{b} a K_{1} \exp \left(\frac{c \varrho}{2-\varrho}\right)\right)^{2^{n}} .
$$

Proof: The proof is a direct combination of results contained in [21] and [23].

\subsubsection{Lemmas on the control of the measure}

In the following lemmas, we consider a fixed $\omega^{(0) \top}=\left(\hat{\omega}^{(0) \top}, \tilde{\omega}^{(0) \top}\right)$, with $\hat{\omega}^{(0)} \in \mathbb{R}^{r}$ and $\tilde{\omega}^{(0)} \in \mathbb{R}^{s}$. Let $\lambda(\varphi)$ be a function defined on $\mathcal{E} \subset \mathbb{R}^{r+1}$ with range in $\mathbb{C}$, where $\varphi^{\top}=$ $\left(\hat{\omega}^{\top}, \varepsilon\right)$, with $\hat{\omega} \in \mathbb{R}^{r}$ and $\varepsilon \in \mathbb{R}$. We assume that $\lambda$ takes the form:

$$
\lambda(\varphi)=\lambda_{0}+i u \varepsilon+i v^{\top}\left(\hat{\omega}-\hat{\omega}^{(0)}\right)+\tilde{\lambda}(\varphi),
$$

where $\lambda_{0}, u \in \mathbb{C}, v \in \mathbb{C}^{r}$ and, if we denote by $\overline{\mathcal{E}}=\overline{\mathcal{E}}(\bar{\vartheta}):=\left\{\varphi \in \mathcal{E}:\left|\varphi-\varphi^{(0)}\right| \leq \bar{\vartheta}\right\}$, $\varphi^{(0)^{\top}}=\left(\hat{\omega}^{(0)^{\top}}, 0\right)$, then we have that $\mathcal{L}_{\overline{\mathcal{E}}}\{\tilde{\lambda}\} \leq L \bar{\vartheta}$ for certain $L \geq 0$, for all $0 \leq \bar{\vartheta} \leq \vartheta_{0}$. We also assume that $\left|\lambda(\varphi)-\lambda_{0}\right| \leq M\left|\varphi-\varphi^{(0)}\right|$ for all $\varphi \in \overline{\mathcal{E}}\left(\vartheta_{0}\right)$. We remark that the Lipschitz bound for $\tilde{\lambda}$ formulated on a sufficiently smooth function, means that $\tilde{\lambda}$ is of $O_{2}\left(\varphi-\varphi^{(0)}\right)$.

Now, we take $\mu>0, \gamma>r+s-1$ and $0<\delta \leq 1$ to define from $\lambda$ and $\mathcal{E}$ the following "resonant" sets:

$$
\begin{aligned}
\mathcal{R}\left(\varepsilon_{0}, R_{0}\right)= & \left\{\hat{\omega} \in \mathbb{R}^{r}:\left|\hat{\omega}-\hat{\omega}^{(0)}\right| \leq R_{0},\left(\hat{\omega}^{\top}, \varepsilon_{0}\right)^{\top}=\varphi \in \mathcal{E}\right. \text { and } \\
& \left.\exists k \in \mathbb{Z}^{r+s} \backslash\{0\} \text { such that }\left|i k^{\top} \omega+\lambda(\varphi)\right|<\frac{\mu}{|k|_{1}^{\gamma}} \exp \left(-\delta|k|_{1}\right)\right\},
\end{aligned}
$$

for every $\varepsilon_{0} \geq 0$ and $R_{0} \geq 0$, and

$$
\begin{aligned}
\mathcal{A}\left(\varepsilon_{0}, \hat{\omega}\right)= & \left\{\varepsilon \in\left[0, \varepsilon_{0}\right]:\left(\hat{\omega}^{\top}, \varepsilon\right)^{\top}=\varphi \in \mathcal{E}\right. \text { and } \\
& \left.\exists k \in \mathbb{Z}^{r+s} \backslash\{0\} \text { such that }\left|i k^{\top} \omega+\lambda(\varphi)\right|<\frac{\mu}{|k|_{1}^{\gamma}} \exp \left(-\delta|k|_{1}\right)\right\},
\end{aligned}
$$

for every $\hat{\omega} \in \mathbb{R}^{r}$ and $\varepsilon_{0}>0$, where in both cases $\omega \in \mathbb{R}^{r+s}$ is defined from $\varphi^{\top}=\left(\hat{\omega}^{\top}, \varepsilon\right)$ as $\omega^{\top}=\left(\hat{\omega}^{\top}, \hat{\omega}^{(0) \top}\right)$. Note that these sets depend on $\delta$ and $\mu$.

As the purpose of this section is to deal with the measure of these resonant sets, we will always assume we are in the worst case: $\operatorname{Re} \lambda_{0}=0$. When this is not true (this is, when there are no resonances) it is not difficult to see that the sets $\mathcal{R}$ and $\mathcal{A}$ are empty if we are close enough to $\varphi^{(0)}$ (the value of the parameter for the unperturbed system). We want to remark that we are not making any assumption on the values $\operatorname{Im} u$ and $\operatorname{Im} v$. According to the size of the resonant sets the worst case happens when $\operatorname{Im} u=0$ and/or $\operatorname{Im} v=0$. Hence, the proof will be valid in this case, although it is possible to improve the measure estimates assuming that $\operatorname{Im} u \neq 0$ and $\operatorname{Im} v \neq 0$. 
Lemma 15 If we assume that $\left|i k^{\top} \omega^{(0)}+\lambda_{0}\right| \geq \frac{\mu_{0}}{|k|_{1}^{\gamma}}, \gamma>r+s-1$, for all $k \in \mathbb{Z}^{r+s} \backslash\{0\}$, for certain $\mu_{0} \geq 2 \mu$, then, if $K$ is the only positive solution of $\frac{\mu_{0}}{2 K^{\gamma}}=M \max \left\{R_{0}, \varepsilon_{0}\right\}+K R_{0}$, we have:

(i) If $\operatorname{Re} v \notin \mathbb{Z}^{r}$, and $\varepsilon_{0}, R_{0}$ are small enough (condition that depends only on $v, L, \vartheta_{0}$, $\gamma, \mu_{0}$ and $M$ ), then,

$$
\operatorname{mes}\left(\mathcal{R}\left(\varepsilon_{0}, R_{0}\right)\right) \leq 16 \mu\left(2 \sqrt{r} R_{0}\right)^{r-1}(r+s) \hat{K}(v) K^{r+s-1-\gamma} \frac{\exp (-\delta K)}{\delta},
$$

where $\hat{K}(v):=\sup _{\hat{k} \in \mathbb{Z}^{r}}\left\{\frac{1}{|\hat{k}+\operatorname{Re} v|_{2}}\right\}$, being $|\cdot|_{2}$ the Euclidean norm of $\mathbb{R}^{r}$.

(ii) If $u \neq 0$, and $\varepsilon_{0}, R_{0}:=\left|\hat{\omega}-\hat{\omega}^{(0)}\right|$ are small enough (condition that depends only on $u, L, \vartheta_{0}, \gamma, \mu_{0}$ and $\left.M\right)$, then

$$
\operatorname{mes}\left(\mathcal{A}\left(\varepsilon_{0}, \hat{\omega}\right)\right) \leq \frac{16 \mu}{|u|}(r+s) K^{r+s-1-\gamma} \frac{\exp (-\delta K)}{\delta} .
$$

Proof: We prove here the part (i). Similar ideas can be used to prove (ii). To study the measure of $\mathcal{R}\left(\varepsilon_{0}, R_{0}\right)$, we consider the following decomposition:

$$
\mathcal{R}\left(\varepsilon_{0}, R_{0}\right)=\bigcup_{k \in \mathbb{Z}^{r+s} \backslash\{0\}} \mathcal{R}_{k}\left(\varepsilon_{0}, R_{0}\right),
$$

where $\mathcal{R}_{k}\left(\varepsilon_{0}, R_{0}\right)$ is defined as:

$$
\begin{aligned}
\mathcal{R}_{k}\left(\varepsilon_{0}, R_{0}\right)= & \left\{\hat{\omega} \in \mathbb{R}^{r}:\left|\hat{\omega}-\hat{\omega}^{(0)}\right| \leq R_{0},\left(\hat{\omega}^{\top}, \varepsilon_{0}\right)^{\top}=\varphi \in \mathcal{E}\right. \\
& \text { and } \left.\left|i k^{\top} \omega+\lambda(\varphi)\right|<\frac{\mu}{|k|_{1}^{\gamma}} \exp \left(-\delta|k|_{1}\right)\right\} .
\end{aligned}
$$

To compute the measure of these sets, we take $\hat{\omega}^{(1)}, \hat{\omega}^{(2)} \in \mathcal{R}_{k}\left(\varepsilon_{0}, R_{0}\right)$, and we put $\varphi^{(j) \top}=$ $\left(\hat{\omega}^{(j) \top}, \varepsilon_{0}\right)$ and $\omega^{(j) \top}=\left(\hat{\omega}^{(j) \top}, \tilde{\omega}^{(0) \top}\right)$. Then, from $\left|i k^{\top} \omega^{(j)}+\lambda\left(\varphi^{(j)}\right)\right|<\frac{\mu}{|k|_{1}^{\gamma}} \exp \left(-\delta|k|_{1}\right)$, we clearly have that $\left|i \hat{k}^{\top}\left(\hat{\omega}^{(1)}-\hat{\omega}^{(2)}\right)+\lambda\left(\varphi^{(1)}\right)-\lambda\left(\varphi^{(2)}\right)\right|<\frac{2 \mu}{|k|_{1}^{\gamma}} \exp \left(-\delta|k|_{1}\right)$, where we have split $k^{\top}=\left(\hat{k}^{\top}, \tilde{k}^{\top}\right)$, with $\hat{k} \in \mathbb{Z}^{r}$ and $\tilde{k} \in \mathbb{Z}^{s}$. From that, and using the definition of $\lambda(\varphi)$ one obtains:

$$
\left|i(\hat{k}+v)^{\top}\left(\hat{\omega}^{(1)}-\hat{\omega}^{(2)}\right)+\tilde{\lambda}\left(\varphi^{(1)}\right)-\tilde{\lambda}\left(\varphi^{(2)}\right)\right|<\frac{2 \mu}{|k|_{1}^{\gamma}} \exp \left(-\delta|k|_{1}\right) .
$$

Note that the set $\mathcal{R}_{k}$ is a slice of the set of $\hat{\omega}$ such that $\left|\hat{\omega}-\hat{\omega}^{(0)}\right| \leq R_{0}$. To estimate its measure, we are going to take the values $\hat{\omega}^{(1)}$ and $\hat{\omega}^{(2)}$ such that $\hat{\omega}^{(1)}-\hat{\omega}^{(2)}$ is (approximately) perpendicular to the slice, that is, parallel to the vector $\hat{k}+\operatorname{Re} v$. Then, $\operatorname{mes}\left(\mathcal{R}_{k}\right)$ can be bounded by the product of a bound of the value $\left|\hat{\omega}^{(1)}-\hat{\omega}^{(2)}\right|$ by (a bound of ) the measure of the worst (biggest) section of an hyperplane (of codimension 1 ) with the set $\left|\hat{\omega}-\hat{\omega}^{(0)}\right| \leq R_{0}$.

Hence, assuming now that $\hat{\omega}^{(1)}-\hat{\omega}^{(2)}$ is parallel to the vector $\hat{k}+\operatorname{Re} v$, we have:

$$
\begin{aligned}
\left|\hat{\omega}^{(1)}-\hat{\omega}^{(2)}\right|_{2} & =\frac{\left|(\hat{k}+\operatorname{Re} v)^{\top}\left(\hat{\omega}^{(1)}-\hat{\omega}^{(2)}\right)\right|}{|\hat{k}+\operatorname{Re} v|_{2}} \leq \frac{\left|(\hat{k}+v)^{\top}\left(\hat{\omega}^{(1)}-\hat{\omega}^{(2)}\right)\right|}{|\hat{k}+\operatorname{Re} v|_{2}} \leq \\
& \leq \frac{1}{|\hat{k}+\operatorname{Re} v|_{2}}\left(L \max \left\{R_{0}, \varepsilon_{0}\right\}\left|\hat{\omega}^{(1)}-\hat{\omega}^{(2)}\right|+\frac{2 \mu}{|k|_{1}^{\gamma}} \exp \left(-\delta|k|_{1}\right)\right) .
\end{aligned}
$$


In consequence:

$$
\left.\left(1-\frac{L \max \left\{R_{0}, \varepsilon_{0}\right\}}{|\hat{k}+\operatorname{Re} v|_{2}}\right)\left|\hat{\omega}^{(1)}-\hat{\omega}^{(2)}\right|_{2} \leq \frac{2 \mu}{|k|_{1}^{\gamma}} \exp \left(-\delta|k|_{1}\right)\right) \frac{1}{|\hat{k}+\operatorname{Re} v|_{2}} .
$$

So, if $\varepsilon_{0}$ and $R_{0}$ are small enough (independent on $k$ ) we can bound:

$$
\left|\hat{\omega}^{(1)}-\hat{\omega}^{(2)}\right|_{2} \leq \frac{4 \mu}{|k|_{1}^{\gamma}} \exp \left(-\delta|k|_{1}\right) \hat{K}
$$

where we put $\hat{K}=\hat{K}(v)$. From that:

$$
\operatorname{mes}\left(\mathcal{R}_{k}\left(\varepsilon_{0}, R_{0}\right)\right) \leq \frac{4 \mu}{|k|_{1}^{\gamma}} \exp \left(-\delta|k|_{1}\right)\left(2 \sqrt{r} R_{0}\right)^{r-1} \hat{K},
$$

where $2 \sqrt{r} R_{0}$ is a bound for the diameter of the set $\left\{\hat{\omega} \in \mathbb{R}^{r}:\left|\hat{\omega}-\hat{\omega}^{(0)}\right| \leq R_{0}\right\}$. Then, we have:

$$
\operatorname{mes}\left(\mathcal{R}\left(\varepsilon_{0}, R_{0}\right)\right) \leq \sum_{k \in \mathbb{Z}^{r+s} \backslash\{0\}} \frac{4 \mu}{|k|_{1}^{\gamma}} \exp \left(-\delta|k|_{1}\right)\left(2 \sqrt{r} R_{0}\right)^{r-1} \hat{K} .
$$

In fact, in this sum we only need to consider $k \in \mathbb{Z}^{r+s} \backslash\{0\}$ such that $\mathcal{R}_{k}\left(\varepsilon_{0}, R_{0}\right) \neq \emptyset$. Now, let us see that $\mathcal{R}_{k}\left(\varepsilon_{0}, R_{0}\right)$ is empty if $|k|_{1}$ is less than some critical value $K$.

Let $\varphi \in \mathcal{R}_{k}\left(\varepsilon_{0}, R_{0}\right)$, then we can write:

$$
\begin{aligned}
\frac{\mu_{0}}{|k|_{1}^{\gamma}} & \leq\left|i k^{\top} \omega^{(0)}+\lambda_{0}\right| \leq\left|i k^{\top} \omega+\lambda(\varphi)\right|+\left|\lambda(\varphi)-\lambda_{0}\right|+\left|\hat{k}^{\top}\left(\hat{\omega}-\hat{\omega}^{(0)}\right)\right| \leq \\
& \leq \frac{\mu}{|k|_{1}^{\gamma}} \exp \left(-\delta|k|_{1}\right)+M \max \left\{R_{0}, \varepsilon_{0}\right\}+|\hat{k}|_{1}\left|\hat{\omega}-\hat{\omega}^{(0)}\right| \leq \\
& \leq \frac{\mu}{|k|_{1}^{\gamma}}+M \max \left\{R_{0}, \varepsilon_{0}\right\}+|\hat{k}|_{1} R_{0},
\end{aligned}
$$

and then:

$$
\frac{\mu_{0}}{2|k|_{1}^{\gamma}} \leq M \max \left\{R_{0}, \varepsilon_{0}\right\}+|k|_{1} R_{0}
$$

So, in the sum (18), we only need to consider $k \in \mathbb{Z}^{r+s} \backslash\{0\}$ such that $|k|_{1} \geq K$, where $K$ (that depends on $R_{0}$ and $\varepsilon_{0}$ ) is defined in the statement of the lemma. We assume $R_{0}$ and $\varepsilon_{0}$ small enough such that $K \geq 1$. Now, using that $\#\left\{k \in \mathbb{Z}^{r+s}:|k|_{1}=j\right\} \leq 2(r+s) j^{r+s-1}$ and that $\gamma>r+s-1$, we have:

$$
\begin{aligned}
\operatorname{mes}\left(\mathcal{R}\left(\varepsilon_{0}, R_{0}\right)\right) & \leq 4 \mu\left(2 \sqrt{r} R_{0}\right)^{r-1} \hat{K} \sum_{\substack{k \in \mathbb{Z}^{r+s} \backslash\{0\} \\
|k|_{1} \geq K}} \frac{\exp \left(-\delta|k|_{1}\right)}{|k|_{1}^{\gamma}} \leq \\
& \leq 4 \mu\left(2 \sqrt{r} R_{0}\right)^{r-1} \hat{K} \sum_{j \geq K} 2(r+s) j^{r+s-1} \frac{\exp (-\delta j)}{j^{\gamma}} \leq \\
& \leq 8 \mu\left(2 \sqrt{r} R_{0}\right)^{r-1}(r+s) \hat{K} K^{r+s-1-\gamma} \sum_{j \geq K} \exp (-\delta j)= \\
& =8 \mu\left(2 \sqrt{r} R_{0}\right)^{r-1}(r+s) \hat{K} K^{r+s-1-\gamma} \frac{\exp (-\delta K)}{1-\exp (-\delta)} \leq \\
& \leq 16 \mu\left(2 \sqrt{r} R_{0}\right)^{r-1}(r+s) \hat{K} K^{r+s-1-\gamma} \frac{\exp (-\delta K)}{\delta},
\end{aligned}
$$

where we used that $\frac{1}{1-\exp (-\delta)} \leq \frac{2}{\delta}$, if $0<\delta \leq 1$. 
Lemma 16 With the previous notations, we introduce the set

$$
\begin{aligned}
\mathcal{D}\left(R_{0}\right)= & \left\{\hat{\omega} \in \mathbb{R}^{r}:\left|\hat{\omega}-\hat{\omega}^{(0)}\right| \leq R_{0}\right. \text { and } \\
& \left.\exists k \in \mathbb{Z}^{r+s} \backslash\{0\} \text { such that }\left|k^{\top} \omega\right|<\frac{\mu}{|k|_{1}^{\gamma}} \exp \left(-\delta|k|_{1}\right)\right\} .
\end{aligned}
$$

Let us assume $\left|k^{\top} \omega^{(0)}\right| \geq \frac{\mu_{0}}{|k|_{1}^{\gamma}}$ for all $k \in \mathbb{Z}^{r+s} \backslash\{0\}$, for certain $\mu_{0} \geq 2 \mu$. Then, if $R_{0}$ is small enough (depending only on $\gamma$ and $\mu_{0}$ ), one has:

$$
\operatorname{mes}\left(\mathcal{D}\left(R_{0}\right)\right) \leq 8 \mu\left(2 \sqrt{r} R_{0}\right)^{r-1}(r+s) K^{r+s-1-\gamma} \frac{\exp (-\delta K)}{\delta},
$$

being $K=\left(\frac{\mu_{0}}{2 R_{0}}\right)^{\frac{1}{\gamma+1}}$.

Proof: It is similar to the one of lemma 15.

Lemma 17 Let $\mu_{0}>0, \rho>0$ and $1<\varrho<2$ fixed. We put $\sigma_{n}=\frac{6}{\pi^{2} n^{2}}, \delta_{n}=\frac{\sigma_{n} \rho}{18}$ and $\mu_{n}=\mu_{0} \exp \left(-\varrho^{n}\right)$, for all $n \geq 1$. Then, for every $0<\sigma<1$, we have that, if $K$ is big enough (depending only on $\varrho, \rho, \mu_{0}$ and $\sigma$ ),

$$
\sum_{n \geq 1} \mu_{n} \frac{\exp \left(-\delta_{n} K\right)}{\delta_{n}} \leq \exp \left(-K^{\sigma}\right)
$$

Proof: Let $n_{*}(K)=\frac{\ln K}{\ln \ell}$. We remark that $n_{*}(K) \rightarrow+\infty$ as $K \rightarrow+\infty$. Then, if $K$ is big enough one has that, for all $n \geq n_{*}(K)$,

$$
\frac{(n+1)^{2} \exp \left(-\varrho^{n+1}\right)}{n^{2} \exp \left(-\varrho^{n}\right)}=\left(\frac{n+1}{n}\right)^{2} \exp \left(-\varrho^{n}(\varrho-1)\right) \leq \exp \left(-\frac{\varrho-1}{2}\right),
$$

that allows to bound

$$
\sum_{n \geq 1} n^{2} \exp \left(-\varrho^{n}\right) \leq \frac{n_{*}^{2} \exp \left(-\varrho^{n}\right)}{1-\exp \left(-\frac{\varrho-1}{2}\right)}
$$

Hence,

$$
\begin{aligned}
& \sum_{n \geq 1} \mu_{n} \frac{\exp \left(-\delta_{n} K\right)}{\delta_{n}}=\sum_{n \geq 1} \frac{3 \mu_{0} \pi^{2}}{\rho} n^{2} \exp \left(-\varrho^{n}-\frac{K \rho}{3 \pi^{2} n^{2}}\right)= \\
& \quad=\frac{3 \mu_{0} \pi^{2}}{\rho}\left(\sum_{1 \leq n<n_{*}}+\sum_{n \geq n_{*}}\right) n^{2} \exp \left(-\varrho^{n}-\frac{K \rho}{3 \pi^{2} n^{2}}\right) \leq \\
& \quad \leq \frac{3 \mu_{0} \pi^{2}}{\rho} n_{*}^{2} \exp \left(-\frac{K \rho}{3 \pi^{2} n_{*}^{2}}\right) \sum_{n \geq 1} \exp \left(-\varrho^{n}\right)+\frac{3 \mu_{0} \pi^{2}}{\rho} \sum_{n \geq n_{*}} n^{2} \exp \left(-\varrho^{n}\right) \leq \\
& \leq \frac{3 \mu_{0} \pi^{2}}{\rho} n_{*}^{2}\left(\exp (-\varrho) \exp \left(-\frac{K \rho}{3 \pi^{2} n_{*}^{2}}\right)+\exp \left(-\varrho^{n_{*}}\right)\right) \frac{1}{1-\exp \left(-\frac{\varrho-1}{2}\right)} \leq \\
& \quad \leq \exp \left(-K^{\sigma}\right),
\end{aligned}
$$

for any $0<\sigma<1$ if $K$ is big enough. 


\subsection{Iterative lemma}

Here we give the details of a step of the iterative process used to prove Theorem 1 . For that purpose let us consider a Hamiltonian $H=H(\theta, x, I, y, \varphi)$ of the form:

$$
H=\phi(\varphi)+\omega^{\top} I+\frac{1}{2} z^{\top} \mathcal{B}(\varphi) z+\frac{1}{2} \hat{I}^{\top} \mathcal{C}(\theta, \varphi) \hat{I}+H_{*}(\theta, x, \hat{I}, y, \varphi)+\hat{H}(\theta, x, \hat{I}, y, \varphi),
$$

with the same notations as $(5)$ and $\varphi$ was introduced in section 5.2.4. Moreover, given $\varphi \in \mathcal{E}$ we recall the definition of $\omega \in \mathbb{R}^{r+s}$ as $\left.\omega^{\top}=\left(\hat{\omega}^{\top}, \tilde{\omega}^{(0)}\right)^{\top}\right)$, where $\hat{\omega}$ comes from the first $r$ components of $\varphi$ and $\tilde{\omega}^{(0)} \in \mathbb{R}^{s}$ is given by the quasiperiodic time dependence. Let us write

$$
H(\theta, x, I, y, \varphi)=\tilde{\omega}^{(0) \top} \tilde{I}+H^{\star}(\theta, x, \hat{I}, y, \varphi),
$$

where we assume that $H^{*}$ depends on $(\theta, x, \hat{I}, y)$ in an analytic form, it is $2 \pi$-periodic in $\theta$, it depends on $\varphi \in \mathcal{E}$ in a Lipschitz way and, moreover, $\left\langle H_{*}\right\rangle=0$ (see section 2.3 for the definition) for all $\varphi$. This implies that, skipping the term $\hat{H}$ (this is the small perturbation), we have for every $\varphi \in \mathcal{E}$ an invariant $(r+s)$-dimensional reducible torus with basic frequencies $\omega$. Moreover, we also assume that $\mathcal{B}$ and $\mathcal{C}$ are symmetric matrices with $\mathcal{J}_{m}(\mathcal{B})=\mathcal{B}$ and $\operatorname{det} \overline{\mathcal{C}} \neq 0$. Hence, $J_{m} \mathcal{B}$ is a diagonal matrix, with eigenvalues $\lambda(\varphi)^{\top}=\left(\lambda_{1}(\varphi), \ldots, \lambda_{m}(\varphi),-\lambda_{1}(\varphi), \ldots,-\lambda_{m}(\varphi)\right)$, that we asume all different, that gives the normal behaviour around the unpertubed invariant torus.

More concretely, let us assume that the following bounds hold: for the unperturbed part, for every $j, l=1, \ldots, 2 m$ with $j \neq l$, we have $0<\alpha_{1} \leq\left|\lambda_{j}(\varphi)-\lambda_{l}(\varphi)\right|, \alpha_{1} / 2 \leq$ $\left|\lambda_{j}(\varphi)\right| \leq \alpha_{2} / 2$ for all $\varphi \in \mathcal{E}$, and that $\mathcal{L}_{\mathcal{E}}\left\{\lambda_{j}\right\} \leq \beta_{2} / 2$. Moreover, $\left\|(\overline{\mathcal{C}})^{-1}\right\|_{\mathcal{E}} \leq \bar{m}$ and, for certain $\rho>0$ and $R>0,\|\mathcal{C}\|_{\mathcal{E}, \rho} \leq \hat{m}, \mathcal{L}_{\mathcal{E}, \rho}\{\mathcal{C}\} \leq \tilde{m},\left\|H_{*}\right\|_{\mathcal{E}, \rho, R} \leq \hat{\nu}$ and $\mathcal{L}_{\mathcal{E}, \rho, R}\left\{H_{*}\right\} \leq \tilde{\nu}$. Finally, we bound the size of the perturbation $\hat{H}$ by $\|\hat{H}\|_{\mathcal{E}, \rho, R} \leq M$ and $\mathcal{L}_{\mathcal{E}, \rho, R}\left\{H_{*}\right\} \leq L$. To simplify the bounds, we will assume that $M \leq L$.

Lemma 18 (Iterative lemma) Let us consider a Hamiltonian $H$ as the one we have just described above. We assume that we can bound $\rho, R, \alpha_{2}, \beta_{2}, \bar{m}, \hat{m}, \tilde{m}, \hat{\nu}, \tilde{\nu}, M$ and $L$ by certain fixed absolute constants $\rho_{0}, R_{0}, \alpha_{2}^{*}, \beta_{2}^{*}, \bar{m}^{*}, \hat{m}^{*}, \tilde{m}^{*}, \hat{\nu}^{*}, \tilde{\nu}^{*}, M_{0}$ and $L_{0}$, and that for some fixed $R^{*}>0$ and $\alpha_{1}^{*}>0$ we have $R^{*} \leq R$ and $\alpha_{1}^{*} \leq \alpha_{1}$. We assume that for every $\varphi \in \mathcal{E}$, the corresponding $\omega$ verifies $|\omega| \leq \kappa^{*}$, for some fixed $\kappa^{*}>0$. Finally, we also consider fixed $\tilde{\delta}_{0}>0, \gamma>r+s-1$ and $\mu_{0}>0$.

In these conditions, there exist a constant $\hat{N}$, only depending on the fixed constants showed above plus $r, m$ and $s$, such that for every $\delta>0, \hat{\delta}>0$ and $\mu>0$ for which the following three conditions hold,

a) $0<9 \delta<\rho, 0<9 \hat{\delta}<\rho$ and $\delta / \hat{\delta} \leq \tilde{\delta}_{0}$,

b) for every $\varphi \in \mathcal{E}$,

$$
\left|i k^{\top} \omega+l^{\top} \lambda(\varphi)\right| \geq \frac{\mu}{|k|_{1}^{\gamma}} \exp \left(-\delta|k|_{1}\right), \quad k \in \mathbb{Z}^{r+s} \backslash\{0\}, \quad l \in \mathbb{N}^{2 m},|l|_{1} \leq 2,
$$

being $0<\mu \leq \mu_{0}$,

c) $\Theta:=\hat{N} \frac{M}{\delta^{2 \gamma+3} \mu^{2}} \leq 1 / 2$, 
we have that there exist a function $S(\theta, x, \hat{I}, y, \varphi)$, defined for every $\varphi \in \mathcal{E}$, with $\nabla S$ an analytic function with respect to $(\theta, x, \hat{I}, y)$ on $\mathcal{U}_{\rho-8 \delta, R-8 \hat{\delta}}^{r+s, 2 m+r}, 2 \pi$-periodic on $\theta$ and with Lipschitz dependence on $\varphi \in \mathcal{E}$, such that $\|\nabla S\|_{\mathcal{E}, \rho-8 \delta, R-8 \hat{\delta}} \leq \min \{\delta, \hat{\delta}\}$. Moreover, following the notations of section 5.1.2, the canonical change of variables $\Psi^{S}$ is well defined for every $\varphi \in \mathcal{E}$,

$$
\hat{\Psi}^{S}: \mathcal{U}_{\rho-9 \delta, R-9 \hat{\delta}}^{r+s, 2 m+r} \longrightarrow \mathcal{U}_{\rho-8 \delta, R-8 \hat{\delta}}^{r+s, 2 m+r}
$$

and transforms $H$ into

$$
H^{(1)}(\theta, x, I, y, \varphi):=H \circ \Psi^{S}(\theta, x, I, y),
$$

where

$$
H^{(1)}=\phi^{(1)}(\varphi)+\omega^{\top} I+\frac{1}{2} z^{\top} \mathcal{B}^{(1)}(\varphi) z+\frac{1}{2} \hat{I}^{\top} \mathcal{C}^{(1)}(\theta, \varphi) \hat{I}+H_{*}^{(1)}+\hat{H}^{(1)},
$$

with $\left\langle H_{*}^{(1)}\right\rangle=0$, and where $\mathcal{B}^{(1)}$ and $\mathcal{C}^{(1)}$ are symmetric matrices with $\mathcal{J}_{m}\left(\mathcal{B}^{(1)}\right)=\mathcal{B}^{(1)}$. Moreover if we put $R^{(1)}=R-9 \hat{\delta}$ and $\rho^{(1)}=R-9 \delta$, we have the following bounds:

$$
\begin{aligned}
\|\nabla S\|_{\mathcal{E}, \rho-8 \delta, R-8 \hat{\delta}} & \leq \hat{N} \frac{M}{\delta^{2+2 \gamma} \mu^{2}}, & \left\|\phi^{(1)}-\phi\right\|_{\mathcal{E}} & \leq \hat{N} \frac{M}{\delta^{1+\gamma} \mu}, \\
\left\|\mathcal{B}^{(1)}-\mathcal{B}\right\|_{\mathcal{E}} & \leq \hat{N} \frac{M}{\delta^{1+\gamma} \mu}, & \mathcal{L}_{\mathcal{E}}\left\{\mathcal{B}^{(1)}-\mathcal{B}\right\} & \leq \hat{N} \frac{L}{\delta^{2+2 \gamma} \mu^{2}}, \\
\left\|\mathcal{C}^{(1)}-\mathcal{C}\right\|_{\mathcal{E}, \rho^{(1)}} & \leq \hat{N} \frac{M}{\delta^{2+2 \gamma} \mu^{2}}, & \mathcal{L}_{\mathcal{E}, \rho^{(1)}}\left\{\mathcal{C}^{(1)}-\mathcal{C}\right\} & \leq \hat{N} \frac{L}{\delta^{3+3 \gamma} \mu^{3}}, \\
\left\|H_{*}^{(1)}-H_{*}\right\|_{\mathcal{E}, \rho^{(1)}, R^{(1)}} & \leq \hat{N} \frac{M}{\delta^{3+2 \gamma} \mu^{2}}, & \mathcal{L}_{\mathcal{E}, \rho^{(1)}, R^{(1)}}\left\{H_{*}^{(1)}-H_{*}\right\} & \leq \hat{N} \frac{L}{\delta^{4+3 \gamma} \mu^{3}}, \\
\left\|H^{(1)}\right\|_{\mathcal{E}, \rho^{(1)}, R^{(1)}} & \leq \hat{N} \frac{M}{\delta^{6+4 \gamma} \mu^{4}}, & \mathcal{L}_{\mathcal{E}, \rho^{(1)}, R^{(1)}}\left\{\hat{H}^{(1)}\right\} & \leq \hat{N} \frac{M L}{\delta^{7}+5 \gamma \mu^{5}}
\end{aligned}
$$

Proof: The idea is to use the scheme described in section 2.3, to remove the perturbative terms that are an obstruction for the existence of a (reducible) torus with vector of basic frequencies $\omega$ up to first order in the size of the perturbation. Hence, as we described in section 2.3 we expand $\hat{H}$ in power series around $\hat{I}=0, z=0$ to obtain $H=\tilde{\omega}^{(0)} \tilde{I}+H^{*}$, being

$$
H^{*}=a(\theta)+b(\theta)^{\top} z+c(\theta)^{\top} \hat{I}+\frac{1}{2} z^{\top} B(\theta) z+\hat{I}^{\top} E(\theta) z+\frac{1}{2} \hat{I}^{\top} C(\theta) \hat{I}+\Omega(\theta, x, \hat{I}, y),
$$

with $\langle\Omega>=0$, where we have not written explicitly the dependence on $\varphi$. We look for a generating function $S$,

$$
S(\theta, x, \hat{I}, y)=\xi^{\top} \hat{\theta}+d(\theta)+e(\theta)^{\top} z+f(\theta)^{\top} \hat{I}+\frac{1}{2} z^{\top} G(\theta) z+\hat{I}^{\top} F(\theta) z,
$$

with the same properties as the one given in (7). If we want to obtain the transformed Hamiltonian $H^{(1)}$ we need to compute (see section 5.1.2):

$$
H^{* *}=\left\{H^{*}, S\right\}-\frac{\partial S}{\partial \tilde{\theta}} \tilde{\omega}^{(0)} \text {. }
$$

We introduce the decomposition $H^{* *}=H_{1}^{* *}+H_{2}^{* *}$, with

$$
H_{1}^{* *}=\left\{\omega^{\top} I+\frac{1}{2} z^{\top} \mathcal{B} z+\frac{1}{2} \hat{I}^{\top} \mathcal{C} \hat{I}+H_{*}, S\right\}-\frac{\partial S}{\partial \tilde{\theta}} \tilde{\omega}^{(0)},
$$


and $H_{2}^{* *}=\{\hat{H}, S\}$. Then, we want to select $S$ such that $H+H_{1}^{* *}$ takes the form

$$
H+H_{1}^{* *}=\phi^{(1)}(\varphi)+\omega^{\top} I+\frac{1}{2} z^{\top} \mathcal{B}^{(1)}(\varphi) z+\frac{1}{2} \hat{I}^{\top} \mathcal{C}^{(1)}(\theta, \varphi) \hat{I}+H_{*}^{(1)}(\theta, x, \hat{I}, y, \varphi),
$$

and hence, $\hat{H}^{(1)}=H_{2}^{* *}+\Sigma\left(H^{* *}, S\right)$. We can explicitly compute $H_{1}^{* *}$ :

$$
\begin{aligned}
H_{1}^{* *}= & \left(\frac{1}{2} \frac{\partial}{\partial \hat{\theta}}\left(\hat{I}^{\top} \mathcal{C} \hat{I}\right)+\frac{\partial H_{*}}{\partial \hat{\theta}}\right)(f+F z)-\left(\hat{I}^{\top} \mathcal{C}+\frac{\partial H_{*}}{\partial \hat{I}}\right)\left(\frac{\partial S}{\partial \hat{\theta}}\right)^{\top}+ \\
& +\left(z^{\top} \mathcal{B}+\frac{\partial H_{*}}{\partial z}\right) J_{m}\left(e+G z+F^{\top} \hat{I}\right)-\frac{\partial S}{\partial \theta} \omega .
\end{aligned}
$$

Then, it is not difficult to see that equation (22) leads to equations $\left(e q_{1}\right)-\left(e q_{5}\right)$ given in section 2.3 , replacing $\omega^{(0)}$ by $\omega$, and that

$$
\begin{gathered}
\phi^{(1)}=\phi-\hat{\omega}^{\top} \xi, \\
\mathcal{B}^{(1)}=\mathcal{J}_{m}\left(B^{*}\right), \\
\mathcal{C}^{(1)}=\mathcal{C}+\left[\hat{I}^{\top}\left(\frac{1}{2} \frac{\partial \mathcal{C}}{\partial \hat{\theta}} f-\mathcal{C}\left(\frac{\partial f}{\partial \hat{\theta}}\right)^{\top}\right) \hat{I}-\frac{\partial H_{*}}{\partial \hat{I}}\left(\xi+\left(\frac{\partial d}{\partial \hat{\theta}}\right)^{\top}\right)+\frac{\partial H_{*}}{\partial z} J_{m} e\right]_{(\hat{I}, \hat{I})}, \\
\left.H_{*}^{(1)}=\Omega+H_{1}^{* *}-<H_{1}^{* *}\right\rangle .
\end{gathered}
$$

We will prove that, from the Diophantine bounds of (20), it is possible to construct a convergent expression for $S$, and to obtain suitable bounds for the transformed Hamiltonian.

The first step is to bound the solutions of $\left(e q_{1}\right)-\left(e q_{5}\right)$, using lemmas of section 5.2.1. In what follows, $\hat{N}$ denotes a constant that bounds all the expressions depending on all the absolute fixed constants of the statement of the lemma, and its value is redefined several times during the proof in order to simplify the notation. Moreover, sometimes we do not write explicitly the dependence on $\varphi$, but all the bounds hold for all $\varphi \in \mathcal{E}$. First, we remark that using the bounds on $\hat{H}$, and from lemmas 2 and 8 we can bound $\|\cdot\|_{\mathcal{E}, \rho}$ and $\mathcal{L}_{\mathcal{E}, \rho}\{$.$\} of a-\phi, b, c-\hat{\omega}, B-\mathcal{B}, E$ and $C-\mathcal{C}$ by $\hat{N} M$ and $\hat{N} L$ respectively, with an $\hat{N}$ that only depends on $R^{*}, r$ and $m$. We recall that from the expressions of section 2.3 the solutions of $\left(e q_{1}\right)-\left(e q_{5}\right)$ are unique, and for them we have (working here for a fixed $\varphi \in \mathcal{E}):$

$\left(e q_{1}\right)$ From the expression of $d$ as a function of the coefficients of the Fourier expansion of $a$, it is clear that if we use the bounds of the denominators given by the Diophantine conditions of (20) and lemma 1 we can see that:

$$
|d|_{\rho-\chi} \leq\left(\frac{\gamma}{(\chi-\delta) \exp (1)}\right)^{\gamma} \frac{|\tilde{a}|_{\rho}}{\mu}
$$

if $\rho>\chi>\delta$, and then using that $|\tilde{a}|_{\rho} \leq|a-\phi|_{\rho}$, we can write:

$$
|d|_{\rho-\chi} \leq \hat{N} \frac{M}{(\chi-\delta)^{\gamma} \mu}
$$


$\left(e q_{2}\right)$ We have for $e$

$$
|e|_{\rho-\chi} \leq\left(\frac{2}{\alpha_{1}^{*}}+\left(\frac{\gamma}{(\chi-\delta) \exp (1)}\right)^{\gamma} \frac{1}{\mu}\right)|b|_{\rho},
$$

for all $\rho>\chi>\delta$. Consequently:

$$
|e|_{\rho-\chi} \leq \hat{N} \frac{M}{(\chi-\delta)^{\gamma} \mu}
$$

$\left(e q_{3}\right)$ First we bound $\xi$ :

$$
\begin{aligned}
|\xi| & =\left|(\overline{\mathcal{C}})^{-1} \overline{\mathcal{C}} \xi\right| \leq\left|(\overline{\mathcal{C}})^{-1}\right||\overline{\mathcal{C}} \xi| \leq \bar{m}^{*}\left|\bar{c}-\hat{\omega}-\mathcal{C}\left(\frac{\partial d}{\partial \hat{\theta}}\right)^{\top}\right|_{\rho-\chi} \leq \\
& \leq \bar{m}^{*}\left(|c-\hat{\omega}|_{\rho}+\left|\mathcal{C}\left(\frac{\partial d}{\partial \hat{\theta}}\right)^{\top}\right|_{\rho-\chi}\right) \leq \bar{m}^{*}\left(\hat{N} M+|\mathcal{C}|_{\rho} \frac{2|d|_{\rho-\chi / 2}}{\chi \exp (1)}\right)
\end{aligned}
$$

where $\rho \geq \chi>2 \delta$. Hence,

$$
|\xi| \leq \hat{N} \frac{M}{(\chi-2 \delta)^{\gamma+1} \mu},
$$

for all $\rho \geq \chi>2 \delta$. Then, for $c^{*}$ we have:

$$
\begin{aligned}
\left|c^{*}\right|_{\rho-\chi} & \leq\left|\tilde{c}-\tilde{\mathcal{C}} \xi-\mathcal{C}\left(\frac{\partial d}{\partial \hat{\theta}}\right)^{\top}+\overline{\mathcal{C}\left(\frac{\partial d}{\partial \hat{\theta}}\right)^{\top}}\right|_{\rho-\chi} \leq \\
& \leq|\tilde{c}|_{\rho}+|\mathcal{C}|_{\rho}\left(|\xi|+\left|\left(\frac{\partial d}{\partial \hat{\theta}}\right)^{\top}\right|_{\rho-\chi}\right) \leq \\
& \leq|c-\hat{\omega}|_{\rho}+\hat{m}^{*}\left(|\xi|+\frac{2|d|_{\rho-\chi / 2}}{\chi \exp (1)}\right) \leq \hat{N} \frac{M}{(\chi-2 \delta)^{\gamma+1} \mu} .
\end{aligned}
$$

Hence, if $\rho>\chi>3 \delta$,

$$
|f|_{\rho-\chi} \leq\left(\frac{3 \gamma}{(\chi-3 \delta) \exp (1)}\right)^{\gamma} \frac{\left|c^{*}\right|_{\rho-2 \chi / 3}}{\mu} \leq \hat{N} \frac{M}{(\chi-3 \delta)^{2 \gamma+1} \mu^{2}} .
$$

$\left(e q_{4}\right)$ From the definition of $B^{*}$ given in (8), we have

$$
\begin{aligned}
\left|B^{*}-\mathcal{B}\right|_{\rho-\chi} \leq & |B-\mathcal{B}|_{\rho-\chi}+ \\
& +\left|\left[\frac{\partial H_{*}}{\partial \hat{I}}\left(\xi+\left(\frac{\partial d}{\partial \hat{\theta}}\right)^{\top}\right)\right]_{(z, z)}\right|_{\rho-\chi}+\left|\left[\frac{\partial H_{*}}{\partial z} J_{m} \epsilon\right]_{(z, z)}\right|_{\rho-\chi} \leq \\
\leq & \hat{N} M+(2 m+1) r \frac{\left|H_{*}\right|_{\rho, R}}{\left(R^{*}\right)^{3}}\left(|\xi|+\frac{2|d|_{\rho-\chi / 2}}{\chi \exp (1)}\right)+24 m^{2} \frac{\left|H_{*}\right|_{\rho, R}}{\left(R^{*}\right)^{3}}|e|_{\rho-\chi},
\end{aligned}
$$

and then

$$
\left|B^{*}-\mathcal{B}\right|_{\rho-\chi} \leq \hat{N} \frac{M}{(\chi-2 \delta)^{\gamma+1} \mu}
$$


if $\rho>\chi>2 \delta$, and the same bound holds for $\left|B^{* *}\right|_{\rho-\chi}$ (see (10)). Lemma 1 allows to bound

$$
|G|_{\rho-\chi} \leq\left(\frac{1}{\alpha_{1}^{*}}+\left(\frac{3 \gamma}{(\chi-3 \delta) \exp (1)}\right)^{\gamma} \frac{1}{\mu}\right) 2 m\left|B^{* *}\right|_{\rho-2 \chi / 3}
$$

with $\rho>\chi>3 \delta$. Hence,

$$
|G|_{\rho-\chi} \leq \hat{N} \frac{M}{(\chi-3 \delta)^{2 \gamma+1} \mu^{2}} .
$$

$\left(e q_{5}\right)$ If $\rho>\chi>2 \delta$, we have for $E^{*}$ defined in $(9)$ :

$$
\begin{aligned}
\left|E^{*}\right|_{\rho-\chi} \leq & |E|_{\rho-\chi}+\left|\mathcal{C}\left(\frac{\partial e}{\partial \hat{\theta}}\right)^{\top}\right|_{\rho-\chi}+\left|\left[\frac{\partial H_{*}}{\partial \hat{I}}\left(\xi+\left(\frac{\partial d}{\partial \hat{\theta}}\right)^{\top}\right)\right]_{(\hat{I}, z)}\right|_{\rho-\chi}+ \\
& +\left|\left[\frac{\partial H_{*}}{\partial z} J_{m} e\right]_{(\hat{I}, z)} \leq \hat{\rho-\chi}\right|_{\rho} \leq \hat{N} M+|\mathcal{C}|_{\rho} 2 m \frac{2|e|_{\rho-\chi / 2}}{\chi \exp (1)}+ \\
& +4 m r \frac{\left|H_{*}\right|_{\rho, R}}{\left(R^{*}\right)^{3}}\left(|\xi|+\frac{2|d|_{\rho-\chi / 2}}{\chi \exp (1)}\right)+8 m^{2} \frac{\left|H_{*}\right|_{\rho, R}}{\left(R^{*}\right)^{3}}|e|_{\rho-\chi} .
\end{aligned}
$$

Then,

$$
\left|E^{*}\right|_{\rho-\chi} \leq \hat{N} \frac{M}{(\chi-2 \delta)^{\gamma+1} \mu}
$$

Now, if $\rho>\chi>3 \delta$,

$$
|F|_{\rho-\chi} \leq 2 m\left(\frac{2}{\alpha_{1}^{*}}+\left(\frac{3 \gamma}{(\chi-3 \delta) \exp (1)}\right)^{\gamma} \frac{1}{\mu}\right)\left|E^{*}\right|_{\rho-2 \chi / 3},
$$

that implies

$$
|F|_{\rho-\chi} \leq \hat{N} \frac{M}{(\chi-3 \delta)^{2 \gamma+1} \mu^{2}}
$$

Now, we repeat the same process to bound the Lipschitz constants for the solutions of these equations. For that purpose, we will also need the results of lemmas 7 and 8 to work with the different Lipschitz dependences. We remark that, for the different denominators, we can bound:

$$
\mathcal{L}_{\mathcal{E}}\left\{i k^{\top} \omega+l^{\top} \lambda\right\} \leq|k|_{1}+\frac{\beta_{2}^{*}}{2}|l|_{1},
$$

for every $k \in \mathbb{Z}^{r+s}, l \in \mathbb{N}^{2 m},|l|_{1} \leq 2$. Moreover, we will also use the hypothesis $M \leq L$ to simplify the bounds. Then we have:

$\left(e q_{1}\right)$ We need to take into account the $\varphi$ dependence for all the functions, and so for $d$ we have

$$
d(\theta, \varphi)=\sum_{k \in \mathbb{Z}^{r+s} \backslash\{0\}} \frac{a_{k}(\varphi)}{i k^{\top} \omega} \exp \left(i k^{\top} \theta\right) .
$$

Then, using lemma 7 and $\mathcal{L}_{\mathcal{E}, \rho}\{\tilde{a}\} \leq \mathcal{L}_{\mathcal{E}, \rho}\{a-\phi\}$, one obtains

$$
\begin{aligned}
\mathcal{L}_{\mathcal{E}, \rho-\chi}\{d\} & \leq\left(\frac{\gamma}{(\chi-\delta) \exp (1)}\right)^{\gamma} \frac{\mathcal{L}_{\mathcal{E}, \rho}\{\tilde{a}\}}{\mu}+\left(\frac{2 \gamma+1}{(\chi-2 \delta) \exp (1)}\right)^{2 \gamma+1} \frac{\|\tilde{a}\|_{\mathcal{E}, \rho}}{\mu^{2}} \leq \\
& \leq \hat{N} \frac{L}{(\chi-2 \delta)^{2 \gamma+1} \mu^{2}},
\end{aligned}
$$


for every $\rho>\chi>2 \delta$.

$\left(e q_{2}\right)$

$$
\begin{aligned}
\mathcal{L}_{\mathcal{E}, \rho-\chi}\{e\} \leq & \left(\frac{\gamma}{(\chi-\delta) \exp (1)}\right)^{\gamma} \frac{\mathcal{L}_{\mathcal{E}, \rho}\{b\}}{\mu}+\left(\frac{2 \gamma+1}{(\chi-2 \delta) \exp (1)}\right)^{2 \gamma+1} \frac{\|b\|_{\mathcal{E}, \rho}}{\mu^{2}}+ \\
& +\left(\frac{2 \gamma}{(\chi-2 \delta) \exp (1)}\right)^{2 \gamma} \frac{\|b\|_{\mathcal{E}, \rho}}{\mu^{2}} \frac{\beta_{2}^{*}}{2}+\frac{2}{\alpha_{1}^{*}} \mathcal{L}_{\mathcal{E}, \rho}\{b\}+\frac{4}{\left(\alpha_{1}^{*}\right)^{2}}\|b\|_{\mathcal{E}, \rho} \frac{\beta_{2}^{*}}{2} \leq \\
\leq & \hat{N} \frac{L}{(\chi-2 \delta)^{2 \gamma+1} \mu^{2}},
\end{aligned}
$$

if $\rho>\chi>2 \delta$.

$\left(e q_{3}\right)$ If $\rho \geq \chi>3 \delta$, we have:

$$
\begin{aligned}
\mathcal{L}_{\mathcal{E}}\{\xi\} \leq & \mathcal{L}_{\mathcal{E}}\left\{(\overline{\mathcal{C}})^{-1}\right\}\left\|\bar{c}-\hat{\omega}-\overline{\mathcal{C}\left(\frac{\partial d}{\partial \hat{\theta}}\right)^{\top}}\right\|_{\mathcal{E}, \rho-\chi}+ \\
& +\left\|(\overline{\mathcal{C}})^{-1}\right\|_{\mathcal{E} \mathcal{L}_{\mathcal{E}, \rho-\chi}}\left\{\bar{c}-\hat{\omega}-\overline{\mathcal{C}\left(\frac{\partial d}{\partial \hat{\theta}}\right)^{\top}}\right\} \leq \hat{N} \frac{L}{(\chi-3 \delta)^{2 \gamma+2} \mu^{2}},
\end{aligned}
$$

where we have used that, from lemma 6 ,

$$
\mathcal{L}_{\mathcal{E}}\left\{(\overline{\mathcal{C}})^{-1}\right\} \leq\left\|(\overline{\mathcal{C}})^{-1}\right\|_{\mathcal{E}}^{2} \mathcal{L}_{\mathcal{E}}\{\overline{\mathcal{C}}\} \leq\left(\bar{m}^{*}\right)^{2} \mathcal{L}_{\mathcal{E}, \rho}\{\mathcal{C}\}
$$

and also that

$$
\mathcal{L}_{\mathcal{E}, \rho-\chi}\left\{\left(\frac{\partial d}{\partial \hat{\theta}}\right)^{\top}\right\} \leq \frac{3}{\chi \exp (1)} \mathcal{L}_{\mathcal{E}, \rho-2 \chi / 3}\{d\},
$$

and $\mathcal{L}_{\mathcal{E}, \rho-\chi}\{\bar{c}-\hat{\omega}\} \leq \mathcal{L}_{\mathcal{E}, \rho}\{c-\hat{\omega}\}$. Then, if $\rho>\chi>3 \delta$, using that $\mathcal{L}_{\mathcal{E}, \rho-\chi}\{\tilde{c}\} \leq$ $\mathcal{L}_{\mathcal{E}, \rho-\chi}\{c-\hat{\omega}\}$, one has

$$
\mathcal{L}_{\mathcal{E}, \rho-\chi}\left\{c^{*}\right\} \leq \mathcal{L}_{\mathcal{E}, \rho-\chi}\{\tilde{c}\}+\mathcal{L}_{\mathcal{E}, \rho-\chi}\{\tilde{\mathcal{C}} \xi\}+\mathcal{L}_{\mathcal{E}, \rho-\chi}\left\{\mathcal{C}\left(\frac{\partial d}{\partial \hat{\theta}}\right)^{\top}\right\} \leq \hat{N} \frac{L}{(\chi-3 \delta)^{2 \gamma+2} \mu^{2}}
$$

Hence,

$$
\begin{aligned}
\mathcal{L}_{\mathcal{E}, \rho-\chi}\{f\} \leq & \left(\frac{3 \gamma}{(\chi-3 \delta) \exp (1)}\right)^{\gamma} \frac{\mathcal{L}_{\mathcal{E}, \rho-2 \chi / 3}\left\{c^{*}\right\}}{\mu}+ \\
& +\left(\frac{2(2 \gamma+1)}{(\chi-6 \delta) \exp (1)}\right)^{2 \gamma+1} \frac{\left\|c^{*}\right\|_{\mathcal{E}, \rho-2 \chi / 3}}{\mu^{2}} \leq \hat{N} \frac{L}{(\chi-6 \delta)^{3 \gamma+2} \mu^{3}},
\end{aligned}
$$

if $\rho>\chi>6 \delta$.

$\left(e q_{4}\right)$ We first bound:

$$
\begin{aligned}
\mathcal{L}_{\mathcal{E}, \rho-\chi}\left\{B^{*}-\mathcal{B}\right\} \leq & \mathcal{L}_{\mathcal{E}, \rho-\chi}\{B-\mathcal{B}\}+\mathcal{L}_{\mathcal{E}, \rho-\chi}\left\{\left[\frac{\partial H_{\star}}{\partial \hat{I}}\left(\xi+\left(\frac{\partial d}{\partial \hat{\theta}}\right)^{\top}\right)\right]_{(z, z)}\right\}+ \\
& +\mathcal{L}_{\mathcal{E}, \rho-\chi}\left\{\left[\frac{\partial H_{*}}{\partial z} J_{m} \epsilon\right]_{(z, z)}\right\} \leq \hat{N} \frac{L}{(\chi-3 \delta)^{2 \gamma+2} \mu^{2}},
\end{aligned}
$$


if $\rho>\chi>3 \delta$, and the same bound holds for $\mathcal{L}_{\mathcal{E}, \rho-\chi}\left\{B^{* *}\right\}$. This implies

$$
\begin{aligned}
\mathcal{L}_{\mathcal{E}, \rho-\chi}\{G\} \leq & (2 m-1) \frac{1}{\alpha_{1}^{*}} \mathcal{L}_{\mathcal{E}, \rho-\chi}\left\{B^{* *}\right\}+(2 m-1) \frac{1}{\left(\alpha_{1}^{*}\right)^{2}}\left\|B^{* *}\right\|_{\mathcal{E}, \rho-\chi} \beta_{2}^{*}+ \\
& +2 m\left(\frac{3 \gamma}{(\chi-3 \delta) \exp (1)}\right)^{\gamma} \frac{\mathcal{L}_{\mathcal{E}, \rho-2 \chi / 3}\left\{B^{* *}\right\}}{\mu}+ \\
& +2 m\left(\frac{3(2 \gamma+1)}{(\chi-6 \delta) \exp (1)}\right)^{2 \gamma+1} \frac{\left\|B^{* *}\right\|_{\mathcal{E}, \rho-2 \chi / 3}}{\mu^{2}}+ \\
& +2 m\left(\frac{6 \gamma}{(\chi-6 \delta) \exp (1)}\right)^{2 \gamma} \frac{\left\|B^{* *}\right\|_{\mathcal{E}, \rho-2 \chi / 3}}{\mu^{2}} \beta_{2}^{*} \leq \\
\leq & \hat{N} \frac{L}{(\chi-6 \delta)^{3 \gamma+2} \mu^{3}},
\end{aligned}
$$

if $\rho>\chi>6 \delta$.

(eq5) From the definition of $E^{*}$,

$$
\begin{aligned}
\mathcal{L}_{\mathcal{E}, \rho-\chi}\left\{E^{*}\right\} \leq & \mathcal{L}_{\mathcal{E}, \rho-\chi}\{E\}+\mathcal{L}_{\mathcal{E}, \rho-\chi}\left\{\left[\frac{\partial H_{*}}{\partial \hat{I}}\left(\xi+\left(\frac{\partial d}{\partial \hat{\theta}}\right)^{\top}\right)\right]_{(\hat{I}, z)}\right\}+ \\
& +\mathcal{L}_{\mathcal{E}, \rho-\chi}\left\{\mathcal{C}\left(\frac{\partial e}{\partial \hat{\theta}}\right)^{\top}\right\}+\mathcal{L}_{\mathcal{E}, \rho-\chi}\left\{\left[\frac{\partial H_{*}}{\partial z} J_{m} \epsilon\right]_{(\hat{I}, z)}\right\} \leq \\
\leq & \hat{N} \frac{L}{(\chi-4 \delta)^{2 \gamma+2} \mu^{2}},
\end{aligned}
$$

if $\rho>\chi>4 \delta$. Hence, if now $\rho>\chi>6 \delta$, we can bound:

$$
\begin{aligned}
\mathcal{L}_{\mathcal{E}, \rho-\chi}\{F\} \leq & 2 m \frac{2}{\alpha_{1}^{*}} \mathcal{L}_{\mathcal{E}, \rho-\chi}\left\{E^{*}\right\}+2 m \frac{4}{\left(\alpha_{1}^{*}\right)^{2}}\left\|E^{*}\right\|_{\mathcal{E}, \rho-\chi} \frac{\beta_{2}^{*}}{2}+ \\
& +2 m\left(\frac{3 \gamma}{(\chi-3 \delta) \exp (1)}\right)^{\gamma} \frac{\mathcal{L}_{\mathcal{E}, \rho-2 \chi / 3}\left\{E^{*}\right\}}{\mu}+ \\
& +2 m\left(\frac{3(2 \gamma+1)}{(\chi-6 \delta) \exp (1)}\right)^{2 \gamma+1} \frac{\left\|E^{*}\right\|_{\mathcal{E}, \rho-2 \chi / 3}}{\mu^{2}}+ \\
& +2 m\left(\frac{6 \gamma}{(\chi-6 \delta) \exp (1)}\right)^{2 \gamma} \frac{\left\|E^{*}\right\|_{\mathcal{E}, \rho-2 \chi / 3}}{\mu^{2}} \frac{\beta_{2}^{*}}{2} \leq \\
& + \\
\leq & \hat{N} \frac{L}{(\chi-6 \delta)^{3 \gamma+2} \mu^{3}} .
\end{aligned}
$$

Before bounding the transformed Hamiltonian, let us check that the change given by the generating function $S$ is well defined. First, we have that:

$$
\|\nabla S\|_{\mathcal{E}, \rho-\chi, R} \leq \hat{N} \frac{M}{(\chi-4 \delta)^{2 \gamma+2} \mu^{2}},
$$

and that

$$
\mathcal{L}_{\mathcal{E}, \rho-\chi, R}\{\nabla S\} \leq \hat{N} \frac{L}{(\chi-7 \delta)^{3 \gamma+3} \mu^{3}}
$$


provided that $\rho>\chi>7 \delta$. If we select $\chi=8 \delta$, and if we consider (28), we have a bound of the type:

$$
\|\nabla S\|_{\mathcal{E}, \rho-8 \delta, R-8 \hat{\delta}} \leq \hat{N} \frac{M}{\delta^{2 \gamma+2} \mu^{2}}
$$

Before continuing, let us ask to the quantity

$$
\left(r+(r+2 m) \exp (1) \max \left\{1, \tilde{\delta}_{0}\right\}\right) \hat{N} \frac{M}{\delta^{2 \gamma+3} \mu^{2}},
$$

to be bounded by $1 / 2$ (this will be used in (30) and (31)). We can bound expression (29) by $\Theta:=\hat{N} \frac{M}{\delta^{2 \gamma+3} \mu^{2}}$ with a redefinition of $\hat{N}$, and hence, this condition (on the size of $M$ ) can be reduced to $\Theta \leq 1 / 2$. We note that $\hat{N}$ only depends on the absolute constants given in the hypothesis of the lemma, and this is, in fact, the assumption on the size of $\Theta$ that appears in those hypothesis. In what follows, we will redefine the value of $\hat{N}$ in order to meet a few more conditions, but this redefinition will not change the fact that the final $\hat{N}$ is an absolute constant.

From this last bound one obtains,

$$
\|\nabla S\|_{\mathcal{E}, \rho-8 \delta, R-8 \hat{\delta}} \leq \frac{\Theta \delta}{\max \left\{1, \tilde{\delta}_{0}\right\}} \leq \min \left\{\delta, \delta / \tilde{\delta}_{0}\right\} \leq \min \{\delta, \hat{\delta}\}
$$

and

$$
\Delta_{\delta, \hat{\delta}} \exp (1)\|\nabla S\|_{\mathcal{E}, \rho-8 \delta, R-8 \hat{\delta}} \leq \Theta,
$$

where we use the definition of $\Delta_{\delta, \hat{\delta}}$ given in (17).

From $(30)$ and lemma 13 we have that $\Psi^{S}$ is well defined (for every $\varphi \in \mathcal{E}$ ), according to (21). From (31) and lemma 10 we can bound the expression of $\Sigma\left(H^{* *}, S\right)$ that appear in the transformed Hamiltonian,

$$
\left\|\Sigma\left(H^{* *}, S\right)\right\|_{\mathcal{E}, \rho^{(1)}, R^{(1)}} \leq\left(\sum_{j \geq 1} \frac{1}{j+1}\left(\frac{1}{2}\right)^{j-1}\right) \Theta\left\|H^{* *}\right\|_{\mathcal{E}, \rho-8 \delta, R-8 \hat{\delta}^{\bullet}}
$$

and, similarly, for the Lipschitz constant we can use lemma 12 to produce

$$
\mathcal{L}_{\mathcal{E}, \rho^{(1)}, R^{(1)}}\left\{\Sigma\left(H^{* *}, S\right)\right\} \leq \sum_{j \geq 1}\left(\frac{1}{j+1}\left(\Delta_{\delta, \hat{\delta}} \exp (1)\right)^{j} \hat{F}_{2}^{j-1}\left(j \hat{L}_{2} \hat{F}_{1}+\hat{L}_{1} \hat{F}_{2}\right)\right)
$$

with $\hat{F}_{1}=\left\|H^{* *}\right\|_{\mathcal{E}, \rho-8 \delta, R-8 \hat{\delta}}, \quad \hat{F}_{2}=\|\nabla S\|_{\mathcal{E}, \rho-8 \delta, R-8 \hat{\delta}}, \hat{L}_{1}=\mathcal{L}_{\mathcal{E}, \rho-8 \delta, R-8 \hat{\delta}}\left\{H^{* *}\right\}$ and $\hat{L}_{2}=$ $\mathcal{L}_{\mathcal{E}, \rho-8 \delta, R-8 \hat{\delta}}\{\nabla S\}$. Then

$$
\begin{aligned}
\mathcal{L}_{\mathcal{E}, \rho^{(1)}, R^{(1)}}\left\{\Sigma\left(H^{* *}, S\right)\right\} \leq & \left(\sum_{j \geq 1} \frac{j}{j+1}\left(\frac{1}{2}\right)^{j-1}\right) \Delta_{\delta, \hat{\delta}} \exp (1) \hat{L}_{2} \hat{F}_{1}+ \\
& +\left(\sum_{j \geq 1} \frac{1}{j+1}\left(\frac{1}{2}\right)^{j-1}\right) \Delta_{\delta, \hat{\delta}} \exp (1) \hat{L}_{1} \hat{F}_{2} .
\end{aligned}
$$

With those expressions, to bound $\Sigma\left(H^{* *}, S\right)$ is reduced to bound $H^{* *}$, with the only remark that the sums $\sum_{j \geq 1} \frac{1}{j+1} \nu^{j-1}=-\frac{\ln (1-\nu)+\nu}{\nu^{2}}$ and $\sum_{j \geq 1} \frac{j}{j+1} \nu^{j-1}=\frac{(1-\nu) \ln (1-\nu)+\nu}{\nu^{2}(1-\nu)}$ are well defined for $\nu=1 / 2$. 
Now, we can bound the transformed Hamiltonian. From the bounds that come from the solutions of $\left(e q_{1}\right)-\left(e q_{5}\right)$ we have:

$$
\left\|H_{1}^{* *}\right\|_{\mathcal{E}, \rho-\chi, R-\eta} \leq \hat{N} \frac{M}{(\chi-4 \delta)^{2 \gamma+3} \mu^{2}} \max \{1, \chi / \eta\}
$$

and

$$
\mathcal{L}_{\mathcal{E}, \rho-\chi, R-\eta}\left\{H_{1}^{* *}\right\} \leq \hat{N} \frac{L}{(\chi-7 \delta)^{3 \gamma+4} \mu^{3}} \max \{1, \chi / \eta\} .
$$

To obtain these bounds, we use the explicit expression of $H_{1}^{* *}$ given in (23), and lemmas 1, 2, 7 and 8 to bound the different partial derivatives. We remark that here we need to use that $|\omega| \leq \kappa^{*}$ for any $\varphi \in \mathcal{E}$. Moreover, from the bound for the Poisson brackets given in lemmas 9 and 11 we have, for $H_{2}^{* *}$,

$$
\left\|H_{2}^{* *}\right\|_{\mathcal{E}, \rho-\chi, R-\eta} \leq \hat{N} \frac{M^{2}}{(\chi-4 \delta)^{2 \gamma+3} \mu^{2}} \max \{1, \chi / \eta\}
$$

and

$$
\mathcal{L}_{\mathcal{E}, \rho-\chi, R-\eta}\left\{H_{2}^{* *}\right\} \leq \hat{N} \frac{L M}{(\chi-7 \delta)^{3 \gamma+4} \mu^{3}} \max \{1, \chi / \eta\} .
$$

The techniques that we use to control the reduction in the different domains when we use Cauchy estimates, are analogous to the ones used in all the previous bounds. Hence, it is clear that we can estimate $H^{* *}$ with an analogous bounds as the ones for $H_{1}^{* *}$.

Finally, using all those bounds and from the explicit expressions of $\phi^{(1)}, \mathcal{B}^{(1)}, \mathcal{C}^{(1)}, H_{1}^{(1)}$ and $\hat{H}^{(1)}$ in $(24)-(27)$ it is not difficult to obtain the final $\hat{N}$ such that all the bounds in the statements of the lemma hold.

\subsection{Proof of the theorem}

We split the proof of the theorem in several parts: in the first one we use one step of the iterative method described in section 2.3 as a linear scheme to reduce the size of the perturbation. Then, we introduce $\hat{\omega}$ as a new parameter to describe the family of lower dimensional tori near the initial one. The next step is to apply the bounds of the iterative scheme given by lemma 18, and we prove the convergence of this scheme for a suitable set of parameters. Finally, we obtain the different estimates on the measure of this set.

\subsubsection{Linear scheme with respect to $\varepsilon$}

We consider the initial Hamiltonian given in the formulation of Theorem 1, and we apply one step of the iterative method described in section 2.3. We remark that from the Diophantine bounds in the statements of the theorem, we can guarantee that this step is possible for small enough values of $\varepsilon$, and that it keeps the initial $C^{2}$ differentiability with respect to $\varepsilon$ on the transformed Hamiltonian. We put $H^{(0)}$ for this Hamiltonian that, if we skip the constant term, looks like:

$$
H^{(0)}=\omega^{(0) \top} I+\frac{1}{2} z^{\top} \mathcal{B}^{(0)}(\varepsilon) z+\frac{1}{2} \hat{I}^{\top} \mathcal{C}^{(0)}(\theta, \varepsilon) \hat{I}+H_{*}^{(0)}(\theta, x, \hat{I}, y, \varepsilon)+\varepsilon^{2} \hat{\mathcal{H}}^{(0)}(\theta, x, \hat{I}, y, \varepsilon),
$$

with the same kind of analytic properties with respect to $(\theta, x, \hat{I}, y)$ as the initial one, in a new domain that is independent on $\varepsilon$ (small enough). We remark that the new matrices 
$\mathcal{B}^{(0)}$ and $\mathcal{C}^{(0)}$ depend on $\varepsilon$, and that $\mathcal{C}^{(0)}$ depends also on $\theta$. Moreover, for $H_{*}$ we do not have the semi-normal form conditions given in $\mathbf{P} 1$ and $\mathbf{P} 2$. As this step comes from a perturbative (linear) method, we have that $\mathcal{B}^{(0)}-\mathcal{B}, \mathcal{C}^{(0)}-\mathcal{C}$ and $H_{*}^{(0)}-H_{*}$ are of $O(\varepsilon)$.

Our aim is to repeat the same iterative scheme. We remark that in the next step and in the ones that follows, we can not guarantee good Diophantine properties for the new eigenvalues of $J_{m} \mathcal{B}^{(0)}$ because this matrix changes at each step of the process. This is the reason that forces us to use parameters to control these eigenvalues. So, we can only work in the set of parameters for which certain Diophantine bounds hold. But before that, we want to introduce a new parameter.

\subsubsection{Introduction of the vector of frequencies as a parameter}

Here we add a new parameter to introduce a family of Hamiltonians $H^{(0)}$. We consider values of $\hat{\omega} \in \mathbb{R}^{r}$ close to $\hat{\omega}^{(0)}$, and for any of these values we perform the change given in (14). So, following the notation introduced in section 5.2.4, we put $\varphi^{\top}=\left(\hat{\omega}^{\top}, \varepsilon\right)$ and then we write this family of Hamiltonians in the following form:

$$
\begin{aligned}
H^{(1)}(\theta, x, I, y, \varphi)= & \tilde{\omega}^{(0) \top} \tilde{I}+\hat{\omega}^{(0) \top}\left(\hat{I}+\mathcal{C}^{-1}\left(\hat{\omega}-\hat{\omega}^{(0)}\right)\right)+\frac{1}{2} z^{\top} \mathcal{B}^{(0)}(\varepsilon) z+ \\
& +\frac{1}{2}\left(\hat{I}+\mathcal{C}^{-1}\left(\hat{\omega}-\hat{\omega}^{(0)}\right)\right)^{\top} \mathcal{C}^{(0)}(\theta, \varepsilon)\left(\hat{I}+\mathcal{C}^{-1}\left(\hat{\omega}-\hat{\omega}^{(0)}\right)\right)+ \\
& +H_{*}^{(0)}\left(\theta, x, \hat{I}+\mathcal{C}^{-1}\left(\hat{\omega}-\hat{\omega}^{(0)}\right), y, \varepsilon\right)+ \\
& +\varepsilon^{2} \mathcal{H}^{(0)}\left(\theta, x, \hat{I}+\mathcal{C}^{-1}\left(\hat{\omega}-\hat{\omega}^{(0)}\right), y, \varepsilon\right),
\end{aligned}
$$

that can be expanded as

$$
H^{(1)}=\phi^{(1)}(\varphi)+\omega^{\top} I+\frac{1}{2} z^{\top} \mathcal{B}^{(1)}(\varphi) z+\frac{1}{2} \hat{I}^{\top} \mathcal{C}^{(1)}(\theta, \varphi) \hat{I}+H_{*}^{(1)}(\theta, x, \hat{I}, y, \varphi)+\hat{H}^{(1)}(\theta, x, \hat{I}, y, \varphi),
$$

(we recall $\omega^{\top}=\left(\hat{\omega}^{\top}, \tilde{\omega}^{(0) \top}\right)$ ) with analogous properties for the different terms as in $(32)$, where we can take $\hat{H}^{(1)}$ of $O_{2}\left(\varphi-\varphi^{(0)}\right), \varphi^{(0)^{\top}}=\left(\hat{\omega}^{(0)^{\top}}, 0\right)$. This comes from the seminormal form structure that we have for $H_{*}$, and the fact that $H_{*}^{(0)}$ is $\varepsilon$-close to $H_{*}$. We also remark that we have differentiable dependence of this Hamiltonian with respect to $\varphi$ (in fact it is analytic with respect to $\hat{\omega}$ ), but since we will work in the following steps on Cantor sets, we can not keep this kind of dependence. So, we replace the differentiable dependence by a Lipschitz one, in the sense given in section 5.1.1. To quantify all these facts, we take $0<\rho \leq 1,0<R \leq 1$ and $0<\vartheta_{1} \leq 1$, such that if we put $\rho^{(1)}=\rho$ and $R^{(1)}=R$, then we have analogous bounds as the ones described in section 5.3 for (19), given by $\rho^{(1)}, R^{(1)}$, and some positive constants $\alpha_{1}^{(1)}, \alpha_{2}^{(1)}, \beta_{2}^{(1)}, \bar{m}^{(1)}, \hat{m}^{(1)}, \tilde{m}^{(1)}, \hat{\nu}^{(1)}$ and $\tilde{\nu}^{(1)}$ on the set $\mathcal{E}^{(1)}=\left\{\varphi \in \mathbb{R}^{r+1}:\left|\varphi-\varphi^{(0)}\right| \leq \vartheta_{1}\right\}$, with respect to the "unperturbed part". For the perturbation $\hat{H}^{(1)}$, if we work with sets of the form $\overline{\mathcal{E}}^{(1)}=\overline{\mathcal{E}}^{(1)}(\bar{\vartheta}):=$ $\left\{\varphi \in \mathcal{E}^{(1)}:\left|\varphi-\varphi^{(0)}\right| \leq \bar{\vartheta}\right\}$, for all $0 \leq \bar{\vartheta} \leq \vartheta_{1}$, we can replace $M$ and $L$ by $N_{1} \bar{\vartheta}^{2}$ and $N_{1} \bar{\vartheta}$, for some $N_{1}>0$. To simplify the following bounds we assume, without loss of generality, that $N_{1} \geq 1$.

Finally, we finish this part with an explicit formulation of the nondegeneracy hypothesis of the normal eigenvalues with respect to the parameters. Let us consider $\mathcal{B}^{(1)}$. By construction, we have that $J_{m} \mathcal{B}^{(1)}$ is a diagonal matrix with the same kind of eigenvalues 
as the matrix $J_{m} \mathcal{B}$ that appears in the statement of the theorem. Then, using the $C^{2}$ differentiability with respect to $\varphi$, we can write its eigenvalues as:

$$
\lambda_{j}^{(1)}(\varphi)=\lambda_{j}+i u_{j} \varepsilon+i v_{j}^{\top}\left(\hat{\omega}-\hat{\omega}^{(0)}\right)+\tilde{\lambda}_{j}^{(1)}(\varphi)
$$

for $j=1, \ldots, 2 m$, with $u_{j} \in \mathbb{C}$ and $v_{j} \in \mathbb{C}^{r}$, and where the Lipschitz constant of $\tilde{\lambda}_{j}^{(1)}$ on $\overline{\mathcal{E}}^{(1)}$ is of $O(\bar{\vartheta})$. Then, those generic nondegeneracy conditions are:

NDC For any $j$ such that $\operatorname{Re} \lambda_{j}=0$, we have $u_{j} \neq 0$ and $\operatorname{Re}\left(v_{j}\right) \notin \mathbb{Z}^{r}$. Moreover, if we define $u_{j, l}=u_{j}-u_{l}$ and $v_{j, l}=v_{j}-v_{l}$, we have these same conditions for $u_{j, l}$ and $v_{j, l}$ for any $j \neq l$ such that $\operatorname{Re}\left(\lambda_{j}-\lambda_{l}\right)=0$.

Note that we have used the $C^{2}$ dependence on $\varphi$ to ensure that the Lipschitz constant of $\tilde{\lambda}_{j}^{(1)}$ on $\overline{\mathcal{E}}^{(1)}$ is $O(\bar{\vartheta})$. If the dependence is $C^{1}$ we can only say that this constant is $o(\bar{\vartheta})$. Nevertheless, it is still possible in this case to derive the same results as in the $C^{2}$ case, but the details are more tedious.

The nondegeneracy conditions with respect to $\varepsilon$ are the same ones used in [23] to study the quasiperiodic perturbations of elliptic fixed points, and the nondegeneracy conditions with respect to the $\hat{\omega}$-dependence are analogous to the ones appeared in [26] and [9], but in those cases they were formulated for an unperturbed system having an $r$-dimensional analytic family of $r$-dimensional reducible elliptic tori.

\subsubsection{Inductive part}

We want to apply here the iterative lemma in an inductive form. For this purpose, we define $\sigma_{n}=\frac{6}{\pi^{2} n^{2}}$ for every $n \geq 1$, and we note that $\sum_{n \geq 1} \sigma_{n}=1$. From this definition, we put $\delta_{n}=\frac{\sigma_{n} \rho}{18}, \hat{\delta}_{n}=\frac{\sigma_{n} R}{18}$ and we introduce $\rho^{(n+1)}=\rho^{(n)}-9 \delta_{n}$ and $R^{(n+1)}=R^{(n)}-9 \hat{\delta}_{n}$ for every $n \geq 1$. We also consider a fixed $1<\varrho<2$, to define $\mu_{n}=\exp \left(-\varrho^{n}\right) \mu_{0}$.

We suppose that, at step $n$, we have a Hamiltonian $H^{(n)}$ like $H^{(1)}$ defined for $\varphi$ in a set $\mathcal{E}^{(n)} \subset \mathcal{E}^{(1)}$, with analogous bounds as $H^{(1)}$, replacing the superscript (1) by $(n)$ in the unperturbed part, and with bounds for the perturbation given by $\bar{M}_{n}=M_{n}(\bar{\vartheta})=N_{n} \bar{\vartheta} 2^{n}$ and $\bar{L}_{n}=L_{n}(\bar{\vartheta})=N_{n} \bar{\vartheta}^{2^{n}-1}$, in every set of the form $\overline{\mathcal{E}}^{(n)}(\bar{\vartheta})$, for all $0 \leq \bar{\vartheta} \leq \vartheta_{1}$, being $N_{n}$ independent on $\bar{\vartheta}$. We will show that this is possible if $\vartheta_{1}$ is small enough, with conditions on $\vartheta_{1}$ that are independent on the actual step.

At this point, we define the new set $\mathcal{E}^{(n+1)}$ of good parameters from $\mathcal{E}^{(n)}$ looking at the new Diophantine conditions. We have that $\varphi \in \mathcal{E}^{(n+1)}$ if $\varphi \in \mathcal{E}^{(n)}$ and the following conditions hold:

$$
\left|k^{\top} \omega+l^{\top} \lambda^{(n)}(\varphi)\right| \geq \frac{\mu_{n}}{|k|_{1}^{\gamma}} \exp \left(-\delta_{n}|k|_{1}\right),
$$

for all $k \in \mathbb{Z}^{r+s} \backslash\{0\}, l \in \mathbb{N}^{2 m},|l|_{1} \leq 2$.

Now, we use the iterative lemma for $\varphi \in \mathcal{E}^{(n+1)}$. First we remark that at every step we have $\rho^{(n)} \leq \rho, R / 2 \leq R^{(n)} \leq R, \delta_{n} / \hat{\delta}_{n}=\rho / R, \mu_{n} \leq \mu_{0}$ and, as $\vartheta_{1} \leq 1$, we have that for every $\varphi \in \mathcal{E}^{(1)},|\omega| \leq \max \left\{\left|\hat{\omega}^{(0)}\right|,\left|\hat{\omega}^{(0)}\right|+1\right\}$. Moreover, we assume that we can bound $\alpha_{1}^{(1)} / 2 \leq \alpha_{1}^{(n)}, \alpha_{2}^{(n)} \leq 2 \alpha_{2}^{(1)}, \beta_{2}^{(n)} \leq 2 \beta_{2}^{(1)}, \bar{m}^{(n)} \leq 2 \bar{m}^{(1)}, \hat{m}^{(n)} \leq 2 \hat{m}^{(1)}, \tilde{m}^{(n)} \leq 2 \tilde{m}^{(1)}$, $\hat{\nu}^{(n)} \leq 2 \hat{\nu}^{(1)} \tilde{\nu}^{(n)} \leq 2 \tilde{\nu}^{(1)}$ and $N_{n} \vartheta_{1}^{2^{n}}-2 \leq N_{1}$. We remark that all those bounds hold for $n=1$. Then we consider the constant $\hat{\hat{N}}$, given by the iterative lemma, corresponding to these bounds. 
If we assume that in the actual step we have for $\Theta_{n}:=\hat{N} \frac{N_{n} \vartheta_{1}^{2 n}}{\delta_{n}^{2 \gamma+3} \mu_{n}^{2}}, \Theta_{n} \leq 1 / 2$, then we can apply the iterative lemma to obtain the generating function $S^{(n)}(\theta, x, \hat{I}, y, \varphi)$, with $\left\|\nabla S^{(n)}\right\|_{\mathcal{E}^{(n+1)}, \rho(n)-8 \delta_{n}, R^{(n)}-8 \hat{\delta}_{n}} \leq \min \left\{\delta_{n}, \hat{\delta}_{n}\right\}$. So, in this case we have for $\Psi^{S^{(n)}}$

$$
\hat{\Psi}^{S^{(n)}}: \mathcal{U}_{\rho^{(n+1)}, R^{(n+1)}}^{r+s, 2 m+r} \longrightarrow \mathcal{U}_{\rho^{(n)}-8 \delta_{n}, R^{(n)}-8 \hat{\delta}_{n}}^{r+s, 2 m+r} .
$$

The next step is to bound the transformed Hamiltonian $H^{(n+1)}=H^{(n)} \circ \Psi^{S^{(n)}}$. We work in a set of the form $\overline{\mathcal{E}}^{(n+1)}$, for all $0<\bar{\vartheta} \leq \vartheta_{1}$. From the bounds of the iterative lemma, and the explicit expressions of $\sigma_{n}, \delta_{n}$ and $\mu_{n}$, we can deduce that there exists $\tilde{N}$ (we can assume $\tilde{N} \geq 1$ ) depending on the same constants as $\hat{N}$, such that

$$
\begin{aligned}
& \left\|\nabla S^{(n)}\right\|_{\overline{\mathcal{E}}^{(n+1)}, \rho^{(n)}-8 \delta_{n}, R^{(n)}-8 \hat{\delta}_{n}} \leq \tilde{N} n^{4+4 \gamma}\left(\exp \left(\varrho^{n}\right)\right)^{2} N_{n} \bar{\vartheta}^{2^{n}}, \\
& \left\|\phi^{(n+1)}-\phi^{(n)}\right\|_{\overline{\mathcal{E}}^{(n+1)}} \leq \tilde{N} n^{2+2 \gamma} \exp \left(\varrho^{n}\right) N_{n} \bar{\vartheta}^{2^{n}} \\
& \left\|\mathcal{B}^{(n+1)}-\mathcal{B}^{(n)}\right\|_{\overline{\mathcal{E}}^{(n+1)}} \leq \tilde{N} n^{2+2 \gamma} \exp \left(\varrho^{n}\right) N_{n} \bar{\vartheta}^{2^{n}} \\
& \mathcal{L}_{\overline{\mathcal{E}}(n+1)}\left\{\mathcal{B}^{(n+1)}-\mathcal{B}^{(n)}\right\} \leq \tilde{N} n^{4+4 \gamma}\left(\exp \left(\varrho^{n}\right)\right)^{2} N_{n} \bar{\vartheta}^{2^{n}-1}, \\
& \left\|\mathcal{C}^{(n+1)}-\mathcal{C}^{(n)}\right\|_{\overline{\mathcal{E}}^{(n+1)}, \rho^{(n+1)}} \leq \tilde{N} n^{4+4 \gamma}\left(\exp \left(\varrho^{n}\right)\right)^{2} N_{n} \bar{\vartheta}^{2^{n}} \\
& \mathcal{L}_{\overline{\mathcal{E}}^{(n+1)}, \rho^{(n+1)}}\left\{\mathcal{C}^{(n+1)}-\mathcal{C}^{(n)}\right\} \leq \tilde{N} n^{6+6 \gamma}\left(\exp \left(\varrho^{n}\right)\right)^{3} N_{n} \bar{\vartheta}^{2^{n}-1}, \\
& \left\|H_{*}^{(n+1)}-H_{*}^{(n)}\right\|_{\overline{\mathcal{E}}^{(n+1)}, \rho^{(n+1)}, R^{(n+1)}} \leq \tilde{N} n^{6+4 \gamma}\left(\exp \left(\varrho^{n}\right)\right)^{2} N_{n} \bar{\vartheta}^{2^{n}}, \\
& \mathcal{L}_{\overline{\mathcal{E}}(n+1), \rho^{(n+1)}, R^{(n+1)}}\left\{H_{*}^{(n+1)}-H_{*}^{(n)}\right\} \leq \tilde{N} n^{8+6 \gamma}\left(\exp \left(\varrho^{n}\right)\right)^{3} N_{n} \bar{\vartheta}^{2^{n}-1} \text {, } \\
& \left\|\hat{H}^{(n+1)}\right\|_{\overline{\mathcal{E}}_{(n+1), \rho}^{(n+1)}, R^{(n+1)}} \leq \tilde{N} n^{12+8 \gamma}\left(\exp \left(\varrho^{n}\right)\right)^{4} N_{n}^{2} \bar{\vartheta}^{2^{n+1}}, \\
& \mathcal{L}_{\overline{\mathcal{E}}^{(n+1)}, \rho^{(n+1)}, R^{(n+1)}}\left\{\hat{H}^{(1)}\right\} \leq \tilde{N} n^{14+10 \gamma}\left(\exp \left(\varrho^{n}\right)\right)^{5} N_{n}^{2} \bar{\vartheta}^{2^{n+1}-1} .
\end{aligned}
$$

Moreover, we assume that we can bound $\Theta_{n} \leq \tilde{N} n^{6+4 \gamma}\left(\exp \left(\varrho^{n}\right)\right)^{2} N_{n} \vartheta_{1}^{2^{n}}$, with the same constant $\tilde{N}$. Then, we use all these expressions as a motivation to define $N_{n+1}=$ $\tilde{N} n^{14+10 \gamma}\left(\exp \left(\varrho^{n}\right)\right)^{5} N_{n}^{2}$, for $n \geq 1$. To bound how fast $N_{n+1}$ grows with $n$ and $N_{1}$ we use lemma 14:

$$
N_{n} \leq \frac{1}{\tilde{N}}\left(\left(\frac{5}{3}\right)^{14+10 \gamma} \tilde{N} N_{1} \exp \left(\frac{5 \varrho}{2-\varrho}\right)\right)^{2^{n-1}},
$$

if $n \geq 1$. If we also define $\tilde{N}_{n+1}=\tilde{N} n^{8+6 \gamma}\left(\exp \left(\varrho^{n}\right)\right)^{5} N_{n}$, for $n \geq 1$, we clearly have, using that $N_{1} \geq 1$ and $\tilde{N} \geq 1$, that $\tilde{N}_{n} \leq N_{n}$ for $n \geq 2$.

Now, we have to justify that we can use the iterative lemma in this inductive form when $n \geq 2$. To this end we need to see that the bounds that we have assumed at the step $n$ (to define $\hat{N}$ and to use the iterative lemma) hold at every step if $\vartheta_{1}$ is small enough. So, we note that if $\vartheta_{1}$ is small enough, the following sum:

$$
\sum_{n \geq 1} N_{n+1} \vartheta_{1}^{2^{n}-2}
$$

is bounded by $\hat{N}^{*}$ that depends on $\varrho$ and the same constants as $\hat{N}$. This bound is not difficult to obtain if we look at how fast $\tilde{N}_{n}$ grows. Moreover the same ideas can be used to prove that $N_{n} \vartheta_{1}^{2^{n}-2} \leq N_{1}$, if $n \geq 1$ and $\vartheta_{1}$ is small enough.

Then, we can define $\alpha_{1}^{(n+1)}=\alpha_{1}^{(n)}-2 N_{n+1} \vartheta_{1}^{2^{n}}, \alpha_{2}^{(n+1)}=\alpha_{2}^{(n)}+2 N_{n+1} \vartheta_{1}^{2^{n}}, \beta_{2}^{(n+1)}=\beta_{2}^{(n)}+$ $N_{n+1} \vartheta_{1}^{2^{n}-1}, \hat{m}^{(n+1)}=\hat{m}^{(n)}+N_{n+1} \vartheta_{1}^{2^{n}}, \tilde{m}^{(n+1)}=\tilde{m}^{(n)}+N_{n+1} \vartheta_{1}^{2^{n}-1}, \hat{\nu}^{(n+1)}=\hat{\nu}^{(n)}+N_{n+1} \vartheta_{1}^{2^{n}}$ and $\tilde{\nu}^{(n+1)}=\tilde{\nu}^{(n)}+N_{n+1} \vartheta_{1}^{2^{n}-1}$, that from the convergence of (35) allows to apply another 
step of the iterative scheme, at least for sufficiently small values of $\vartheta_{1}$. Moreover, it is clear that $\Theta_{n} \leq N_{n+1} \vartheta_{1}^{2^{n}} \leq \hat{N}^{*} \vartheta_{1}^{2} \leq 1 / 2$ taken $\vartheta_{1}$ small enough. Then, it only reamains to bound $\bar{m}^{(n+1)}$. For that purpose we first consider the bound $\left\|\overline{\mathcal{C}}^{(n+1)}-\overline{\mathcal{C}}^{(n)}\right\|_{\mathcal{E}^{(n+1)}, \rho^{(n+1)}} \leq N_{n+1} \vartheta_{1}^{2^{n}}$, and then, if we work with a fixed value of $\varphi \in \overline{\mathcal{E}}^{(n+1)}\left(\vartheta_{1}\right)$, we have for any $W \in \mathbb{C}^{r}$ :

$$
\left|\bar{C}^{(n+1)} W\right| \geq\left|\bar{C}^{(n)} W\right|-\left|\left(\bar{C}^{(n+1)}-\bar{C}^{(n)}\right) W\right| \geq\left(\left(\bar{m}^{(n)}\right)^{-1}-N_{n+1} \vartheta_{1}^{2^{n}}\right)|W| .
$$

We note that, from the equivalence $\left|\left(\bar{C}^{(n)}\right)^{-1}\right| \leq \bar{m}^{(n)} \Longleftrightarrow\left|\bar{C}^{(n)} W\right| \geq\left(\bar{m}^{(n)}\right)^{-1}|W|$, for any $W \in \mathbb{C}^{r}$, we can take $\bar{m}^{(n+1)}=\frac{\bar{m}^{(n)}}{1-\bar{m}^{(n)} N_{n+1} \vartheta_{1}^{2 n}}$, provided that $\bar{m}^{(n)} N_{n+1} \vartheta_{1}^{2^{n}}<1$. Then, using this expression we can see that $\bar{m}^{(n)} \leq 2 \bar{m}^{(1)}$ for any $n \geq 1$, if $\vartheta_{1}$ is small enough: if we assume that it holds for $n$, when we compute $\bar{m}^{(n+1)}$ we have that

$$
\bar{m}^{(n)} N_{n+1} \vartheta_{1}^{2^{n}} \leq 2 \bar{m}^{(1)} N_{n+1} \vartheta_{1}^{2^{n}} \leq \frac{1}{2},
$$

if $\vartheta_{1}$ is small enough. Moreover, we have by induction that

$$
\bar{m}^{(n+1)} \leq \bar{m}^{(1)} \prod_{j=1}^{n} \frac{1}{1-2 \bar{m}^{(1)} N_{j+1} \vartheta_{1}^{2^{j}}} .
$$

So, it is clear that, if $\vartheta_{1}$ is small enough,

$$
\sum_{j \geq 1} 2 \bar{m}^{(1)} N_{j+1} \vartheta_{1}^{2^{j}} \leq 2 \bar{m}^{(1)} \hat{N}^{*} \vartheta_{1}^{2} \leq \frac{1}{2} \ln (2),
$$

and hence, if we note that when $0 \leq X \leq 1 / 2$,

$$
\ln \left(\frac{1}{1-X}\right)=\ln \left(1+\frac{X}{1-X}\right) \leq \frac{X}{1-X} \leq 2 X
$$

we can bound $\ln \left(\bar{m}^{(n+1)}\right) \leq \ln \left(\bar{m}^{(1)}\right)+\ln (2)$, that proves $\bar{m}^{(n+1)} \leq 2 \bar{m}^{(1)}$.

\subsubsection{Convergence of the changes of variables}

Now, we are going to prove the convergence of the composition of changes of variables. Let $\mathcal{E}^{*}=\Pi_{n \geq 1} \mathcal{E}^{(n)}$ be the set of $\varphi$ where everything is well defined for all the steps. We consider a fixed $\varphi \in \mathcal{E}^{*}$, but in fact, the results will hold in the whole set $\mathcal{E}^{*}$ provided that $\vartheta_{1}$ is small enough.

We put $\breve{\Psi}^{(n)}=\hat{\Psi}^{(1)} \circ \ldots \circ \hat{\Psi}^{(n)}$ for $n \geq 1$, that goes from $\mathcal{U}_{\rho^{(n+1)}, R^{(n+1)}}^{r+s, 2 m+r}$ to $\mathcal{U}_{\rho, R}^{r+s, 2 m+r}$, where $\hat{\Psi}^{(n)}$ means $\hat{\Psi}^{S^{(n)}}$. Then, if $p>q \geq 1$, we have

$$
\breve{\Psi}^{(p)}-\breve{\Psi}^{(q)}=\sum_{j=q}^{p-1}\left(\breve{\Psi}^{(j+1)}-\breve{\Psi}^{(j)}\right) .
$$

To bound $\breve{\Psi}^{(j+1)}-\breve{\Psi}^{(j)}$, we define $\rho_{j}^{\prime}=\rho^{(j)}-\rho / 4$ and $R_{j}^{\prime}=R^{(j)}-R / 4$, and we put $\hat{\Delta}_{\rho, R}=\frac{1}{\exp (1) \rho}+\frac{r+2 m}{R}$. Now, let us see that

$$
\begin{aligned}
& \left|\breve{\Psi}^{(j+1)}-\breve{\Psi}^{(j)}\right|_{\rho_{j+2}^{\prime}, R_{j+2}^{\prime}}=\left|\hat{\Psi}^{(1)} \circ \ldots \circ \hat{\Psi}^{(j+1)}-\hat{\Psi}^{(1)} \circ \ldots \circ \hat{\Psi}^{(j)}\right|_{\rho_{j+2}^{\prime}, R_{j+2}^{\prime}} \leq \\
\leq & \left(1+4 \hat{\Delta}_{\rho, R}\left|\hat{\Phi}^{(1)}\right|_{\rho(2), R^{(2)}}\right)\left|\hat{\Psi}^{(2)} \circ \ldots \circ \hat{\Psi}^{(j+1)}-\hat{\Psi}^{(2)} \circ \ldots \circ \hat{\Psi}^{(j)}\right|_{\rho_{j+2}^{\prime}, R_{j+2}^{\prime}},
\end{aligned}
$$


where we note $\hat{\Psi}^{(n)}-I d=\hat{\Phi}^{S^{(n)}}:=\hat{\Phi}^{(n)}$, if $n \geq 1$. To prove it, we write $\hat{\Psi}^{(1)} \circ \ldots \circ$ $\hat{\Psi}^{(j)}=\hat{\Psi}^{(2)} \circ \ldots \circ \hat{\Psi}^{(j)}+\hat{\Phi}^{(1)} \circ \hat{\Psi}^{(2)} \circ \ldots \circ \hat{\Psi}^{(j)}$, for every $j \geq 1$, and then we note that can bound $\left|\hat{\Psi}^{(2)} \circ \ldots \circ \hat{\Psi}^{(j+1)}-I d\right|_{\rho_{j+2}^{\prime}, R_{j+2}^{\prime}}$ and $\left|\hat{\Psi}^{(2)} \circ \ldots \circ \hat{\Psi}^{(j)}-I d\right|_{\rho_{j+2}^{\prime}, R_{j+2}^{\prime}}$ by $\min \left\{\rho^{(2)}-\rho_{j+2}^{\prime}-\rho / 4, R^{(2)}-R_{j+2}^{\prime}-R / 4\right\}$. We prove the first bound, the second is analogous. To prove this, we have:

$$
\begin{aligned}
& \left|\hat{\Psi}^{(2)} \circ \ldots \circ \hat{\Psi}^{(j+1)}-I d\right|_{\rho_{j+2}^{\prime}, R_{j+2}^{\prime}} \leq\left|\hat{\Psi}^{(2)} \circ \ldots \circ \hat{\Psi}^{(j+1)}-I d\right|_{\rho(j+2), R^{(j+2)}} \leq \\
& \leq \sum_{l=2}^{j}\left|\hat{\Psi}^{(l)} \circ \ldots \circ \hat{\Psi}^{(j+1)}-\hat{\Psi}^{(l+1)} \circ \ldots \circ \hat{\Psi}^{(j+1)}\right|_{\rho(j+2), R^{(j+2)}}+ \\
& +\left|\hat{\Psi}^{(j+1)}-I d\right|_{\rho(j+2), R^{(j+2)}} \leq \sum_{l=2}^{j+1}\left|\hat{\Phi}^{(l)}\right|_{\rho^{(l+1)}, R^{(l+1)}}=\sum_{l=2}^{j+1} \min \left\{\delta_{l}, \tilde{\delta}_{l}\right\}= \\
& =\min \left\{\rho^{(2)}-\rho^{(j+2)}, R^{(2)}-R^{(j+2)}\right\}=\min \left\{\rho^{(2)}-\rho_{j+2}^{\prime}-\rho / 4, R^{(2)}-R_{j+2}^{\prime}-R / 4\right\},
\end{aligned}
$$

where we have used lemma 3 to bound the norms of the compositions. From that, we can prove (36) from lemma 4 . Now, if we iterate (36) using similar ideas at every step, we produce the bound

$$
\left|\breve{\Psi}^{(j+1)}-\breve{\Psi}^{(j)}\right|_{\rho_{j+2}^{\prime}, R_{j+2}^{\prime}} \leq \prod_{l=1}^{j}\left(1+4 \hat{\Delta}_{\rho, R}\left|\hat{\Phi}^{(l)}\right|_{\rho^{(l+1)}, R^{(l+1)}}\right)\left|\hat{\Phi}^{(j+1)}\right|_{\rho(j+2), R^{(j+2)}} .
$$

So, from lemma 13 ,

$$
\left|\hat{\Phi}^{(n)}\right|_{\rho(n+1), R^{(n+1)}} \leq\left|\nabla S^{(n)}\right|_{\rho(n)}-8 \delta_{n}, R^{(n)}-8 \hat{\delta}_{n} \leq N_{n+1} \vartheta_{1}^{2^{n}}
$$

and if we assume

$$
4 \hat{\Delta}_{\rho, R} \sum_{l \geq 1} N_{l+1} \vartheta_{1}^{2^{l}} \leq 4 \hat{\Delta}_{\rho, R} \hat{N}^{*} \vartheta_{1}^{2} \leq \ln 2
$$

we have that:

$$
\prod_{l=1}^{j}\left(1+4 \hat{\Delta}_{\rho, R} N_{l+1} \vartheta_{1}^{2^{l}}\right) \leq 2, \text { for every } j \geq 1
$$

Hence, using the convergent character of the sum (35), one obtains

$$
\left|\breve{\Psi}^{(p)}-\breve{\Psi}^{(q)}\right|_{\rho / 4, R / 4} \leq \sum_{j \geq q} 2 N_{j+2} \vartheta_{1}^{2^{j+1}} \rightarrow 0, \text { as } p, q \rightarrow+\infty .
$$

This fact allows to define

$$
\hat{\Psi}^{(*)}:=\lim _{n \rightarrow+\infty} \breve{\Psi}^{(n)}
$$

that maps $\mathcal{U}_{\rho / 4, R / 4}^{r+s, 2 m+r}$ into $\mathcal{U}_{\rho, R}^{r+s, 2 m+r}$.

So, as we remark in section 5.1.2 for this kind of canonical transformations that keeps the quasiperiodic time dependence of the Hamiltonian at every step, we only need to show that the final Hamiltonian is well defined to obtain the convergence of the final canonical change $\Psi^{(*)}$, defined as the composition of all the $\Psi^{S^{(n)}}$. This follows immediately from the different bound for the terms of $H^{(n)}$.

Hence, the limit Hamiltonian $H^{(*)}$ takes the form:

$$
H^{(*)}(\theta, x, I, y, \varphi)=\phi^{(*)}(\varphi)+\omega I+\frac{1}{2} z^{\top} \mathcal{B}^{(*)}(\varphi) z+\frac{1}{2} z^{\top} \mathcal{C}^{(*)}(\theta, \varphi) z+H_{*}^{(*)}(\theta, x, \hat{I}, y, \varphi),
$$

with $\left\langle H_{*}^{(*)}\right\rangle=0$, that is, for every $\varphi \in \mathcal{E}^{*}$, we have a $(r+s)$-dimensional reducible torus. 


\subsubsection{Control of the measure}

To prove the assumptions $(a)$ and $(b)$ of Theorem 1 , we only need to control the measure of the set of parameters for which we can prove convergence of the scheme or, in an equivalent form, which is the measure of the different sets that we remove at each step of the iterative method: the key idea is to study the characteritzation of these sets given by the Diophantine conditions of (34). Hence, we only need to look at the eigenvalues of $\mathcal{B}^{(n)}$. From the bounds of the inductive scheme, we have that $\left\|\lambda_{j}^{(n)}-\lambda_{j}^{(1)}\right\|_{\overline{\mathcal{E}}^{(n)}}=O_{2}(\bar{\vartheta})$ and $\mathcal{L}_{\overline{\mathcal{E}}^{(n)}}\left\{\lambda_{j}^{(n)}-\lambda_{j}^{(1)}\right\}=O(\bar{\vartheta})$ for every $j=1, \ldots, 2 m$ and $n \geq 2$, provided that $0<\bar{\vartheta} \leq \vartheta_{1}$, where the constants that give the different $O_{2}(\bar{\vartheta})$ and $O(\bar{\vartheta})$ are independent on $n$ and $j$. Then, from expression (33) we can write:

$$
\lambda_{j}^{(n)}(\varphi)=\lambda_{j}+i u_{j} \varepsilon+i v_{j}^{\top}\left(\hat{\omega}-\hat{\omega}^{(0)}\right)+\tilde{\lambda}_{j}^{(n)}(\varphi),
$$

with $\mathcal{L}_{\overline{\mathcal{E}}^{(n)}}\left\{\tilde{\lambda}_{j}^{(n)}\right\} \leq L \bar{\vartheta}$ and $\left|\lambda_{j}^{(n)}(\varphi)-\lambda_{j}\right| \leq M\left|\varphi-\varphi^{(0)}\right|$, for certain $L$ and $M$ positive. Then, if we use the nondegeneracy conditions of NDC plus the Diophantine assumptions for the frequencies and eigenvalues of the initial torus, the results of $(a)$ and $(b)$ are consequence of lemmas of section 5.2.4. Here we skip any kind of "hyperbolicity" and we assume that we are always in the worst case, that is, we assume all the normal directions to be of elliptic type.

(a) From the bound for the measure of the set $\mathcal{A}$ in lemma 15, we cleary have that if we put in this lemma $\lambda \equiv \lambda_{j}^{(n)}$ or $\lambda \equiv \lambda_{j}^{(n)}-\lambda_{l}^{(n)}, j \neq l$, in the set $\mathcal{E} \equiv \mathcal{E}^{(n)}$ with $\vartheta_{0} \equiv \vartheta_{1}, \mu \equiv \mu_{n}$ and $\delta \equiv \delta_{n}$, we can bound

$$
\operatorname{mes}\left(\overline{\mathcal{I}}^{(n)} \backslash \overline{\mathcal{I}}^{(n+1)}\right)=O\left(K^{r+s-1-\gamma} \mu_{n} \frac{\exp \left(-\delta_{n} K\right)}{\delta_{n}}\right),
$$

with $\overline{\mathcal{I}}^{(n)}=\left\{\varepsilon \in[0, \bar{\varepsilon}]:\left(\hat{\omega}^{(0) \top}, \varepsilon\right)^{\top}=\varphi \in \mathcal{E}^{(n)}\right\}(\bar{\varepsilon}>0$ small enough) and we can take $K$ such that $\frac{\mu_{0}}{2 K^{\gamma}}=M \bar{\varepsilon}$, that is, $K:=K(\bar{\varepsilon})=\left(\frac{\mu_{0}}{2 M \bar{\varepsilon}}\right)^{\frac{1}{\gamma}}$. Then, if we put $\overline{\mathcal{I}}_{*}=\cap_{n>1} \overline{\mathcal{I}}^{(n)}$ we have from the bounds of lemma 17 that for every $0<\sigma<1$, if $K(\bar{\varepsilon})$ is big enough (that is equivalent to take $\bar{\varepsilon}$ small enough) depending on $\sigma$ :

$$
\operatorname{mes}\left([0, \bar{\varepsilon}] \backslash \overline{\mathcal{I}}_{*}\right) \leq \exp \left(-(1 / \bar{\varepsilon})^{\frac{\sigma}{\gamma}}\right) \text {. }
$$

(b) Now we can use the result (i) of lemma 15 plus lemma 16, working on sets of the form $\mathcal{W}^{(n)}\left(\varepsilon_{0}, R_{0}\right)=\left\{\hat{\omega} \in \mathbb{R}^{r}:\left(\hat{\omega}^{\top}, \varepsilon_{0}\right)^{\top}=\varphi \in \mathcal{E}^{(n)}\right\}$. We have

$$
\operatorname{mes}\left(\mathcal{W}^{(n)} \backslash \mathcal{W}^{(n+1)}\right)=O\left(R_{0}^{r-1} K^{r+s-1-\gamma} \mu_{n} \frac{\exp \left(-\delta_{n} K\right)}{\delta_{n}}\right),
$$

where for $K$ we can take (depending on $\varepsilon_{0}$ and $R_{0}$ ), $K=\min \left\{K_{1}, K_{2}\right\}$, with $\frac{\mu_{0}}{2 K_{1}^{\gamma}}=$ $M \max \left\{R_{0}, \varepsilon_{0}\right\}+K_{1} R_{0}$, condition that comes from lemma 15 , and $K_{2}=\left(\frac{\mu_{0}}{2 R_{0}}\right)^{\frac{1}{\gamma+1}}$, that comes from lemma 16. Then, if we take a fixed $0 \leq \varepsilon_{0} \leq R_{0}^{\frac{\gamma}{\gamma+1}}$ (we recall $R_{0} \leq 1$ ) we can obtain a lower bound for $K$ of $O\left(R_{0}^{-\frac{1}{\gamma+1}}\right)$, where the constant that give this order depends only on $\mu_{0}, \gamma$ and $M$. So, if we use lemma 17 we have the desired bound for the measure of the set $\mathcal{W}_{*}\left(\varepsilon_{0}, R_{0}\right)=\cap_{n \geq 1} \mathcal{W}^{(n)}\left(\varepsilon_{0}, R_{0}\right)$ :

$$
\operatorname{mes}\left(\mathcal{V}\left(R_{0}\right) \backslash \mathcal{W}_{*}\left(\varepsilon, R_{0}\right)\right) \leq \exp \left(-\left(1 / R_{0}\right)^{\frac{\sigma}{\gamma+1}}\right),
$$

for every $0<\sigma<1$, if $R_{0}$ is small enough depending on $\sigma$. 


\section{Acknowledgements}

The authors want to thank Rafael del la Llave and Carles Simó for interesting discussions. The research of A. Jorba has been supported by the spanish grant DGICYT PB94-0215, the EC grant ERBCHRXCT940460, and the catalan grant CIRIT GRQ93-1135. The research of J. Villanueva has been supported by the UPC grant PR9409.

\section{References}

[1] Arnol'd V. I.: Proof of a Theorem of A. N. Kolmogorov on the Invariance of QuasiPeriodic Motions under Small Perturbations of the Hamiltonian, Russ. Math. Surveys, 18:5 (1963), pp. 9-36.

[2] Arnol'd V. I.: Instability of Dynamical Systems with Several Degrees of Freedom, Sov. Math. Dokl. 5 (1964), pp. 581-585.

[3] Benettin G., Galgani L., Giorgilli A. and Strelcyn J.M.: A Proof of Kolmogorov's Theorem on Invariant Tori using Canonical Transformations Defined by the Lie Method, Il Nuovo Cimento 79B:2 (1984), pp. 201-223.

[4] Broer H. W., Huitema G. B. and Takens F.: Unfoldings of Quasi-Periodic Tori, Mem. Amer. Math. Soc., 83:421 (1990), pp. 1-81.

[5] Chierchia L., Absolutely Continuous Spectra of Quasiperiodic Schrödinger Operators, J. Math. Phys., 28 (1987), pp. 2891-2898.

[6] Delshams A. and Gutiérrez P.: Effective Stability and KAM Theory, preprint (1995). To appear in J. Diff. Eq.

[7] Delshams A. and Gutiérrez P.: Estimates on Invariant Tori Near an Elliptic Equilibrium Point of a Hamiltonian System. Preprint (1996).

[8] Díez C., Jorba A. and Simó: A Dynamical Equivalent to the Equilateral Libration Points of the Earth-Moon System, Celestial Mechanics, 50 (1991), pp. 13-29.

[9] Eliasson L. H.: Perturbations of Stable Invariant Tori for Hamiltonian Systems, Ann. Sc. Norm. Super. Pisa, Cl. Sci., Ser. IV, 15:1 (1988), pp. 115-147.

[10] Eliasson L. H.: Floquet Solutions for the 1-dimensional Quasi-Periodic Schrödinger Equation, Comm. Math. Phys., 146:3 (1992), pp. 447-482.

[11] Eliasson L. H.: Biasymptotic Solutions of Perturbed Integrable Hamiltonian Systems, Bol. Soc. Bras. Mat., 25:1 (1994), pp. 57-76.

[12] Gómez G., Jorba A., Masdemont J. and Simó C.: A Quasiperiodic Solution as a Substitute of L4 in the Earth-Moon System, Proceedings of the 3rd International Symposiun of Spacecraft Flight Dynamics, ESA Publications Division, ESTEC, Noordwijk, Holland (1991), pp. 35-41. 
[13] Gómez G., Jorba A., Masdemont J. and Simó C.: A Dynamical Systems Approach for the Analysis of Soho Mission, Proceedings of the 3rd International Symposiun of Spacecraft Flight Dynamics, ES A Publications Division, ESTEC, Noordwijk, Holland (1991), pp. 449-454.

[14] Gómez G., Jorba A., Masdemont J. and Simó C.: Study Refinement of SemiAnalytical Halo Orbit Theory, ESOC Contract 8625/89/D/MD(SC), Final Report, (1991).

[15] Gómez G., Jorba A., Masdemont J. and Simó C.: Study of the Transfer from the Earth to a Halo Orbit around the Equilibrium Point $L_{1}$, Celestial Mechanics and Dynamical Astronomy, 56 (1993), pp. 541-562.

[16] Gómez G., Jorba A., Masdemont J. and Simó C.: Study of Poincaré Maps for Orbits Near Lagrangian Points, ESOC contract 9711/91/D/IM(SC), Final Report (1993).

[17] Graff S.: On the Conservation of Hyperbolic Invariant Tori for Hamiltonian Systems, J. Differ. Eq., 15:1 (1974), pp. 1-69.

[18] Johnson R. A. and Sell G. R.: Smoothness of Spectral Subbundles and Reducibility of Quasi-Periodic Linear Differential Systems, Journal of Differential Equations, 41 (1981), pp. 262-288.

[19] Jorba A., Llave R. de la and Zou M.: Lindstedt Series for Lower Dimensional Tori, Hamiltonian Systems with Three or More Degrees of Freedom, C. Simó (Ed.), NATO Adv. Sci. Inst. Ser. C Math. Phys. Sci. Held in S'Agaró, Spain, June 1995. Kluwer, Dordrecht, Holland, to appear in 1996.

[20] Jorba A., Ramírez-Ros R. and Villanueva J.: Effective Reducibility of Quasiperiodic Linear Equations close to Constant Coefficients, to appear in SIAM Journal on Mathematical Analysis.

[21] Jorba A. and Simó C.: On the Reducibility of Linear Differential Equations with Quasiperiodic Coefficients, Journal of Differential Equations, 98:1 (1992), pp. 111124.

[22] Jorba A. and Simó C.: Effective Stability for Periodically Perturbed Hamiltonian Systems, Hamiltonian Mechanics, Integrability and Chaotic Behaviour, J. Seimenis (Ed.), Plenum Press, New York (1994), pp. 245-252.

[23] Jorba A. and Simó C.: On Quasiperiodic Perturbations of Elliptic Equilibrium Points, to appear in SIAM Journal on Mathematical Analysis.

[24] Krikorian R.: Réductibilité des systèmes produits croisés quasi-pèriodiques à valeurs dans des groupes compacts, Ph. D. Thesis, Ecole Polytechnique, Paris (1996).

[25] Llave R. de la and Wayne C. E.: Whiskered and Low Dimensional Tori in Nearly Integrable Hamiltonian Systems, preprint (1989).

[26] Melnikov V.K.: A Family of Conditionally Periodic Solutions of a Hamiltonian System, Soviet Mathematics Doklady, 9:4 (1968), pp. 882-885. 
[27] Moser J.: Convergent Series Expansions for Quasi-Periodic Motions, Math. Ann., 169:1 (1967), pp. 136-176.

[28] Pöschel J.: On Elliptic Lower Dimensional Tori in Hamiltonian Systems, Math. Z., 202:4 (1989), pp. 559-608.

[29] Richardson, D.L.: Analytic Construction of Periodic Orbits about the Collinear Points, Cel. Mech., 22 (1980), pp. 241-253.

[30] Rudnev M. and Wiggins S.: KAM. Theory Near Multiplicity one Resonant Surfaces in Perturbations of a-Priori Stable Hamiltonian Systems, preprint (1996).

[31] Siegel C. and Moser J.K.: Lectures on Celestial Mechanics, Springer-Verlang (1971).

[32] Simó C., Gómez G., Jorba A. and Masdemont J.: The Bicircular Model Near the Triangular Libration Points of the RTBPP, From Newton to Chaos, Edited by A.E. Roy and B.A. Stevens, Plenum Press, New York (1995), pp. 343-370.

[33] Sevryuk M. B.: The Lack-of-Parameters Problem in the KAM Theory Revisited, Hamiltonian Systems with Three or More Degrees of Freedom, C. Simó (Ed.), NATO Adv. Sci. Inst. Ser. C Math. Phys. Sci. Held in S'Agaró, Spain, June 1995. Kluwer, Dordrecht, Holland, to appear in 1996.

[34] Szebehely V.: Theory of Orbits, Academic Press (1967).

[35] Treschev D. V.: The Mechanism of Destruction of Resonance Tori of Hamiltonian Systems, Math. USSR sb. 68:1 (1991), pp. 181-203.

[36] Zehnder E.: Generalized Implicit Function Theorems with Applications to some Small Divisor Problems II, Comm. Pure Appl. Math., 29:1 (1976), pp. 49-111. 